\title{
Yanhusanines A-F, Isoquinoline-Derived Alkaloid Enantiomers from Corydalis yanhusuo and Their Biological Activity
}

Ling-Yan Wang, Bo-Lin Qiu, Huan Xia, Gui-Yang Xia, Biao-Bing Xiao, Jing-Fang Zhang, Wan-Chao Zhong, and Sheng Lin*

Institute of Materia Medica, Chinese Academy of Medical Sciences and Peking Union Medical College (Key Laboratory of Bioactive Substances and Resources Utilization of Chinese Herbal Medicine, Ministry of Education), Beijing 100050, People's Republic of China

\section{Supplementary Information}

* Corresponding authors. Tel: +86-10-83154789. E-mail: 1sznn@imm.ac.cn (S. Lin) 


\section{Contents}

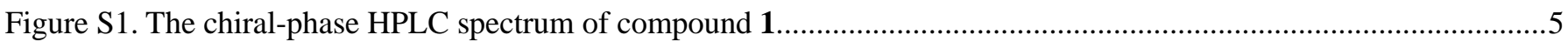

Figure S2. The chiral-phase HPLC spectrum of compound 2

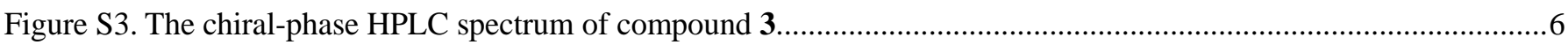

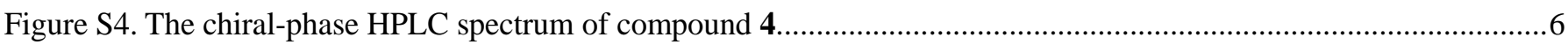

Figure S5. The chiral-phase HPLC spectrum of compound 5

Figure S6. The chiral-phase HPLC spectrum of compound 6

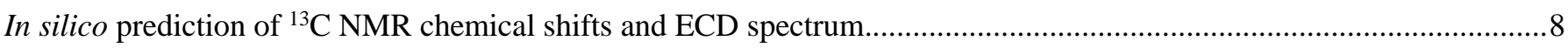

Table S1. Cartesian Coordinates, Relative Energies, and Equilibrium Populations of Low-energy Conformers (>1\%) of $1 R^{*}, 9 S^{*} \mathbf{1}(1 \mathrm{~A})$

Table S2. Cartesian Coordinates, Relative Energies, and Equilibrium Populations of Low-energy Conformers (>1\%) of $1 S^{*}, 9 S^{*}-\mathbf{1}(1 \mathrm{~B})$

Table S3. Cartesian Coordinates, Relative Energies, and Equilibrium Populations of Low-energy Conformers (>1\%) of $9 R^{*}-2$

Table S4. Cartesian Coordinates, Relative Energies, and Equilibrium Populations of Low-energy Conformers (>1\%) of $9 S^{*}-\mathbf{3}$.

Table S5. Cartesian Coordinates, Relative Energies, and Equilibrium Populations of Low-energy Conformers (>1\%) of $9 S^{*}-4$.

Table S6. Cartesian Coordinates, Relative Energies, and Equilibrium Populations of Low-energy Conformers (>1\%) of $9 R *-5$

Table S7. Cartesian Coordinates, Relative Energies, and Equilibrium Populations of Low-energy Conformers (>1\%) of $9 R^{*}-6$

Table S8. Extracted heats and weighting factors of the optimized conformers of $\mathbf{1 A}\left(1 R^{*}, 9 S^{*}-1\right)$ and $\mathbf{1 B}\left(1 S^{*}, 9 S^{*}-1\right)$ at B3LYP/6-311+G(d, p) level in MeOH with PCM model.

Table S9. Extracted heats and weighting factors of the optimized conformers of $9 R^{*-2}$ at B3LYP/6-311+G $(\mathrm{d}, \mathrm{p})$ level in $\mathrm{MeOH}$ with PCM model.

Table S10. Extracted heats and weighting factors of the optimized conformers of $9 S^{*}-\mathbf{3}$ at B3LYP/6-311+G (d, p) level in $\mathrm{MeOH}$ with PCM model.

Table S11. Extracted heats and weighting factors of the optimized conformers of $9 S^{*}-4$ at B3LYP/6-311+G (d, p) level in $\mathrm{MeOH}$ with PCM model.

Table S12. Extracted heats and weighting factors of the optimized conformers of $9 R^{*}-5$ at B3LYP/6-311+G (d, p) level in $\mathrm{MeOH}$ with PCM model.

Table S13. Extracted heats and weighting factors of the optimized conformers of $9 R^{*}-6$ at B3LYP/6-311+G (d, p) level in $\mathrm{MeOH}$ with PCM model.

Table S14. Calculated ${ }^{13} \mathrm{C}$ NMR chemical shifts (ppm) and DP4+ for $1 \mathrm{~A}\left(1 R^{*}, 9 S^{*}-1\right)$ and $1 \mathrm{~B}\left(1 S^{*}, 9 S^{*}-1\right) \ldots$

Figure S7. Correlation plots of experimental ${ }^{13} \mathrm{C}$ NMR chemical shifts versus corresponding calculated ${ }^{13} \mathrm{C}$ NMR chemical shifts for $\mathbf{1}$.

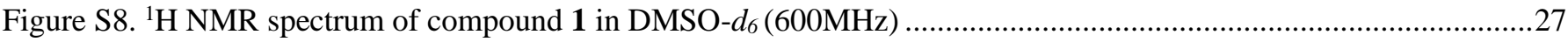

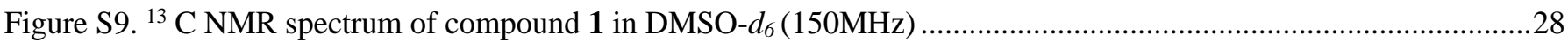

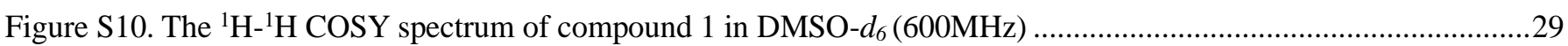

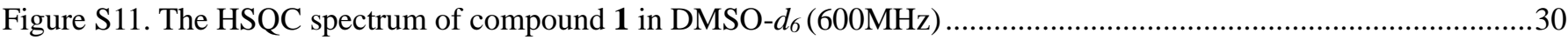

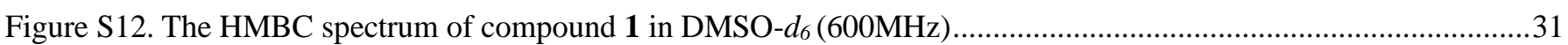

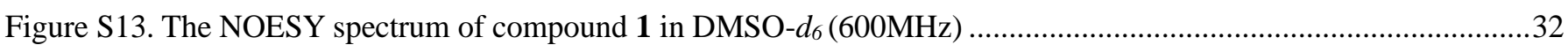

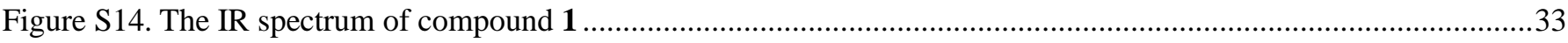

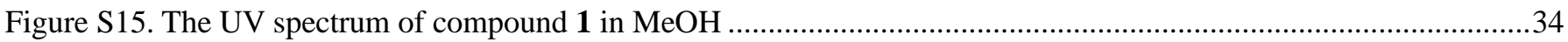

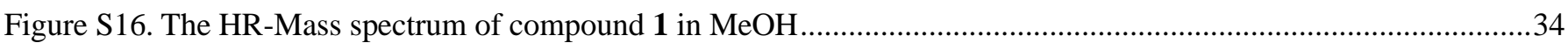




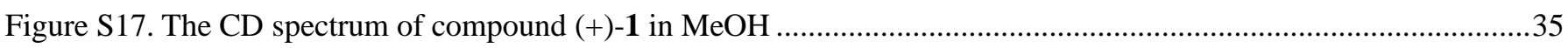

Figure S18. The CD spectrum of compound (-)-1 in $\mathrm{MeOH}$

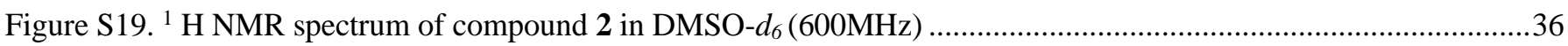

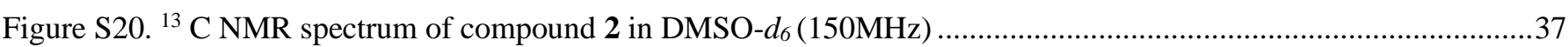

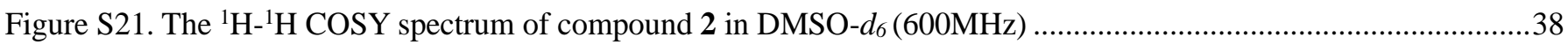

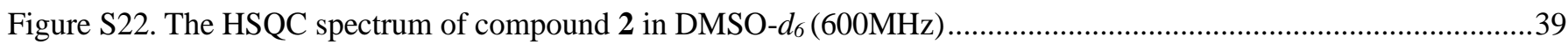

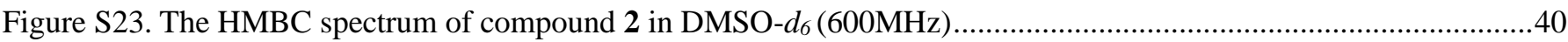

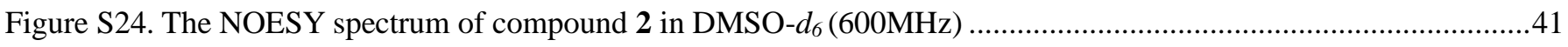

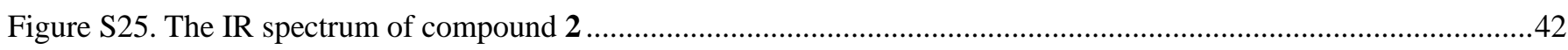

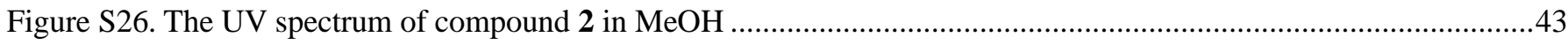

Figure S27. The HR-Mass spectrum of compound 2 in $\mathrm{MeOH}$

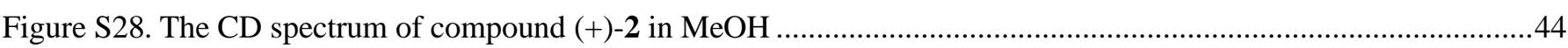

Figure S29. The CD spectrum of compound (-)-2 in $\mathrm{MeOH}$

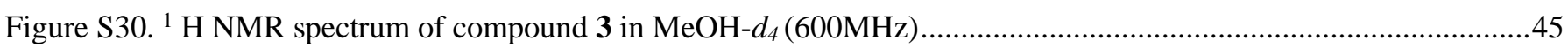

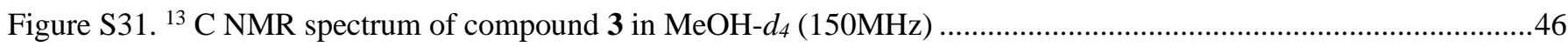

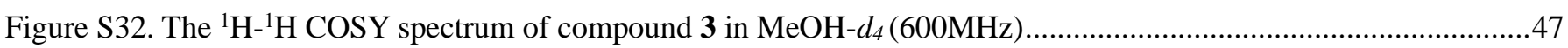

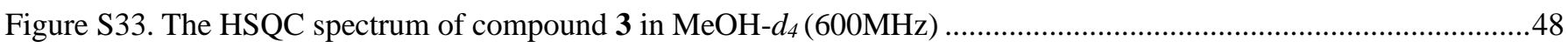

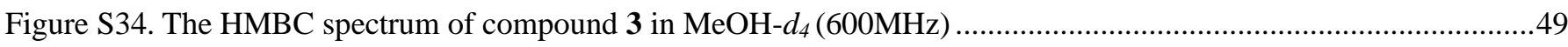

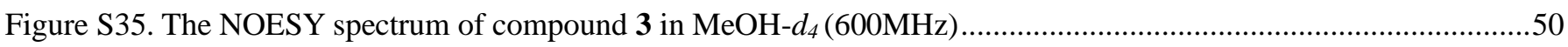

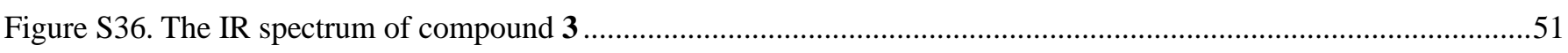

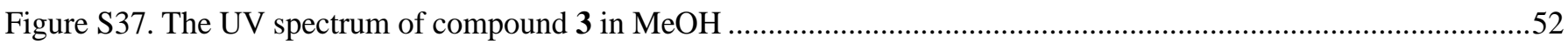

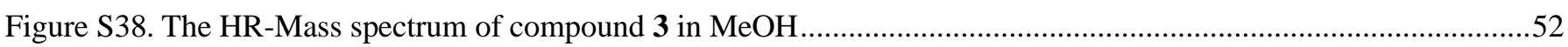

Figure S39. The CD spectrum of compound (+)-3 in $\mathrm{MeOH}$

Figure S40. The CD spectrum of compound (-)-3 in $\mathrm{MeOH}$

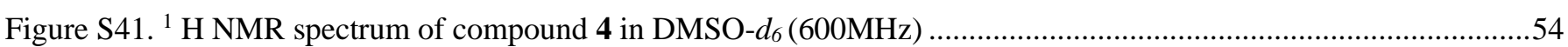

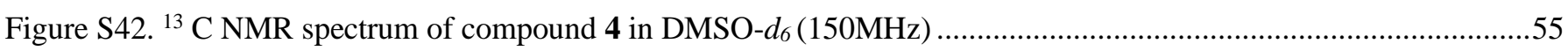

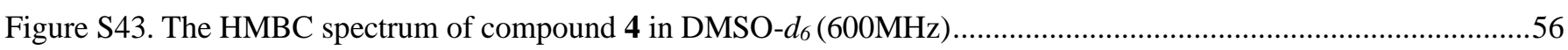

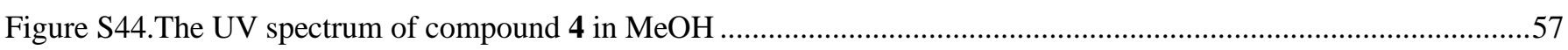

Figure S45.The HR-Mass spectrum of compound 4 in $\mathrm{MeOH}$

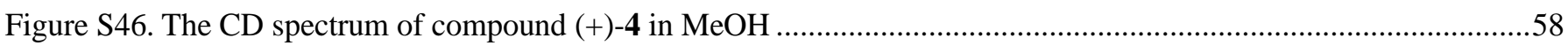

Figure S47. The CD spectrum of compound (-)-4 in $\mathrm{MeOH}$

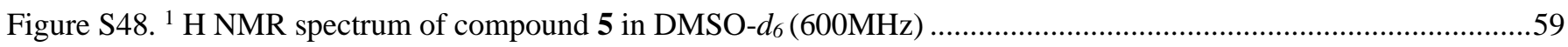

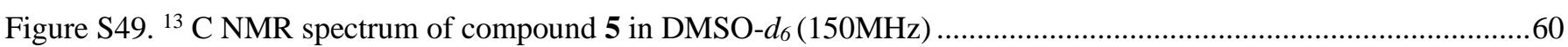

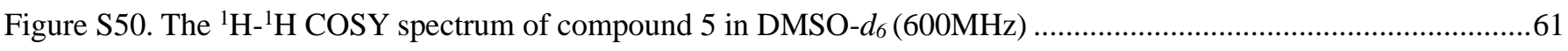

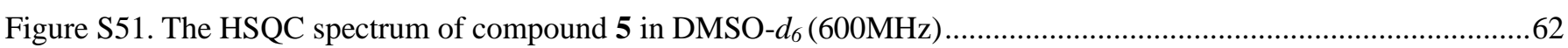

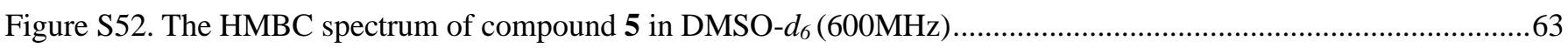

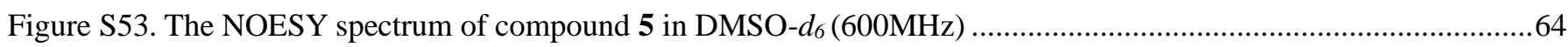

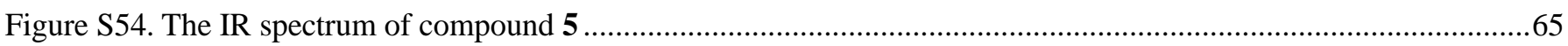

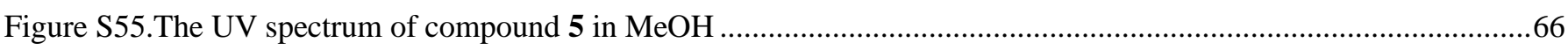

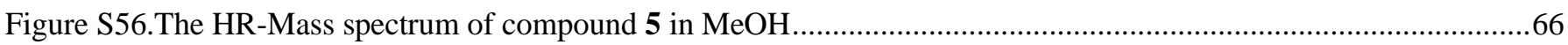

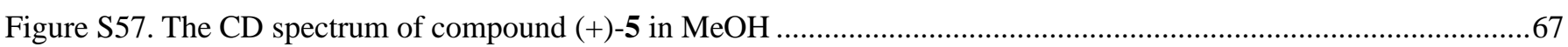

Figure S58. The CD spectrum of compound (-)-5 in $\mathrm{MeOH}$

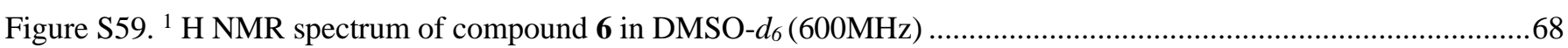

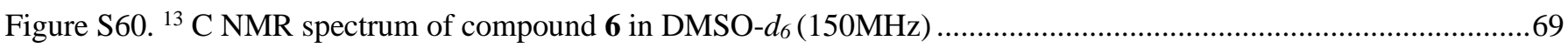

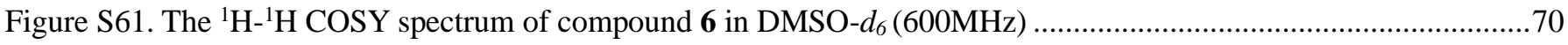

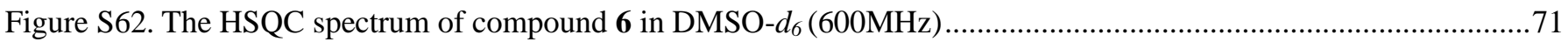

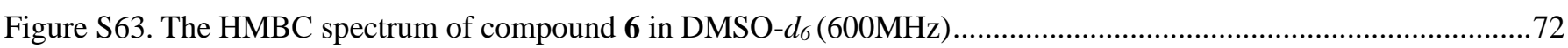


Figure S64. The NOESY spectrum of compound 6 in DMSO- $d_{6}(600 \mathrm{MHz})$ .73

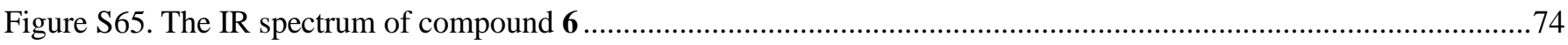

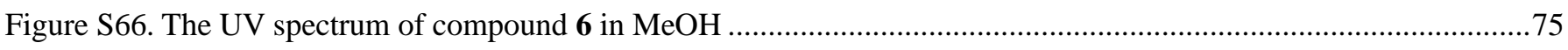

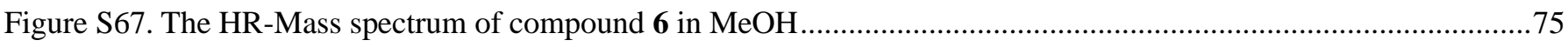

Figure S68. The CD spectrum of compound (+)-6 in $\mathrm{MeOH}$

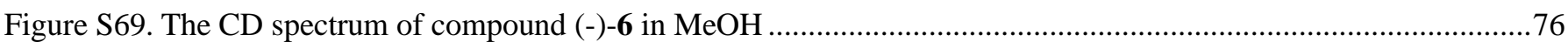




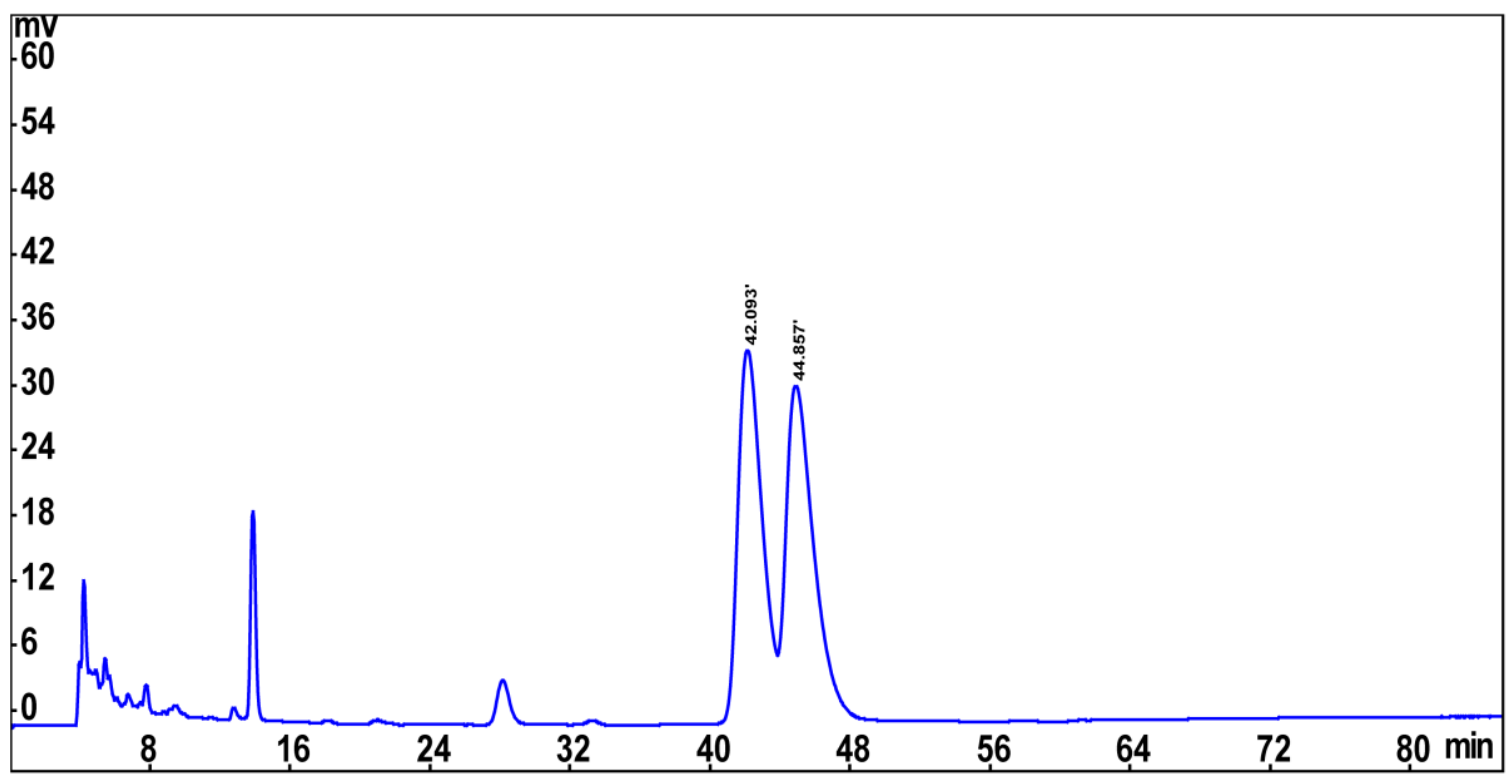

Figure S1. The chiral-phase HPLC spectrum of compound 1

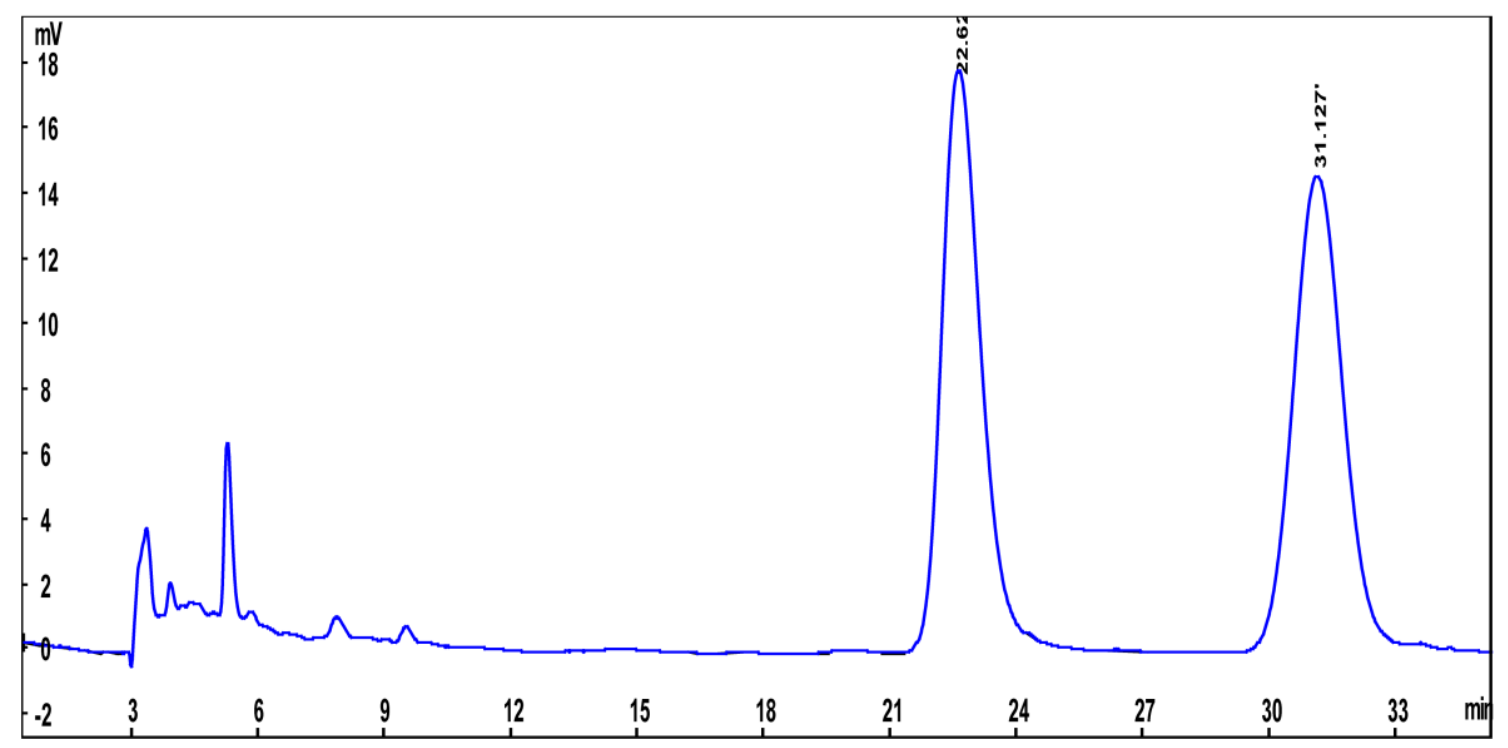

Figure S2. The chiral-phase HPLC spectrum of compound 2 


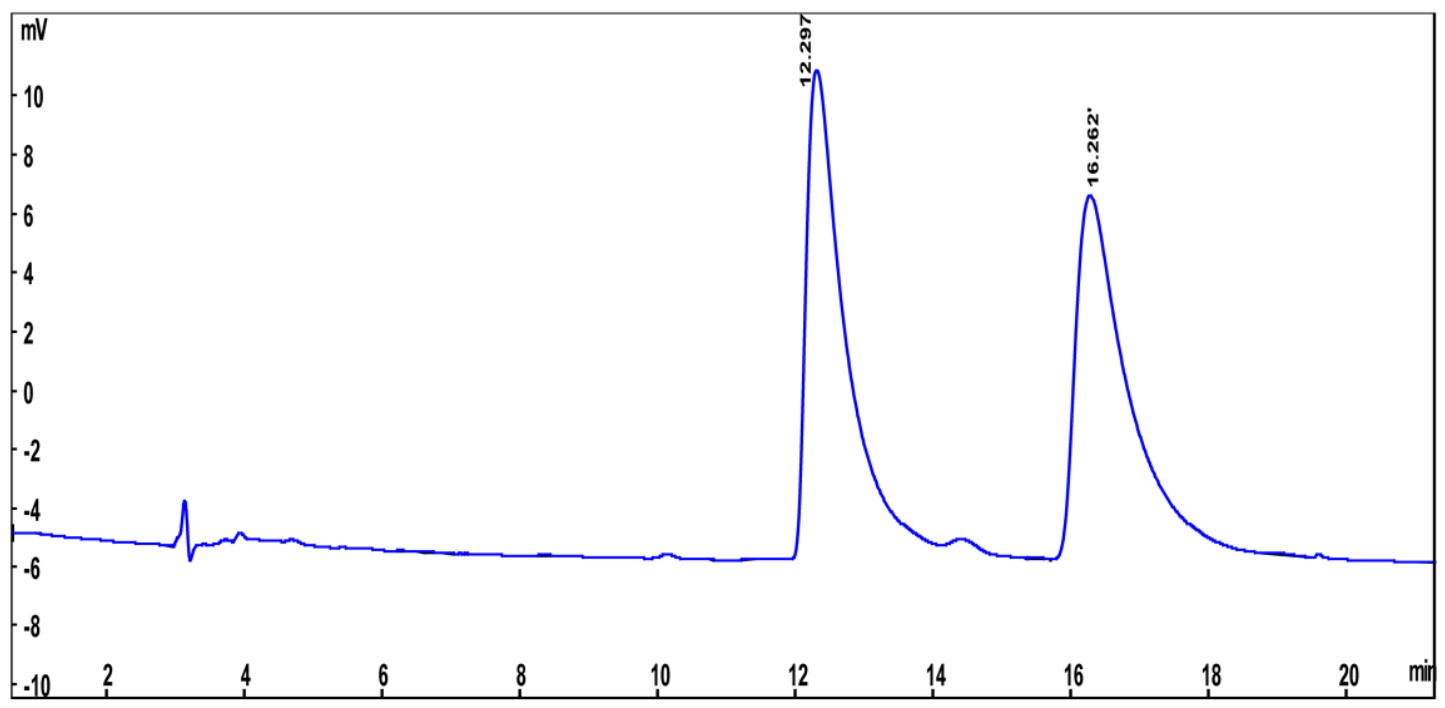

Figure S3. The chiral-phase HPLC spectrum of compound 3

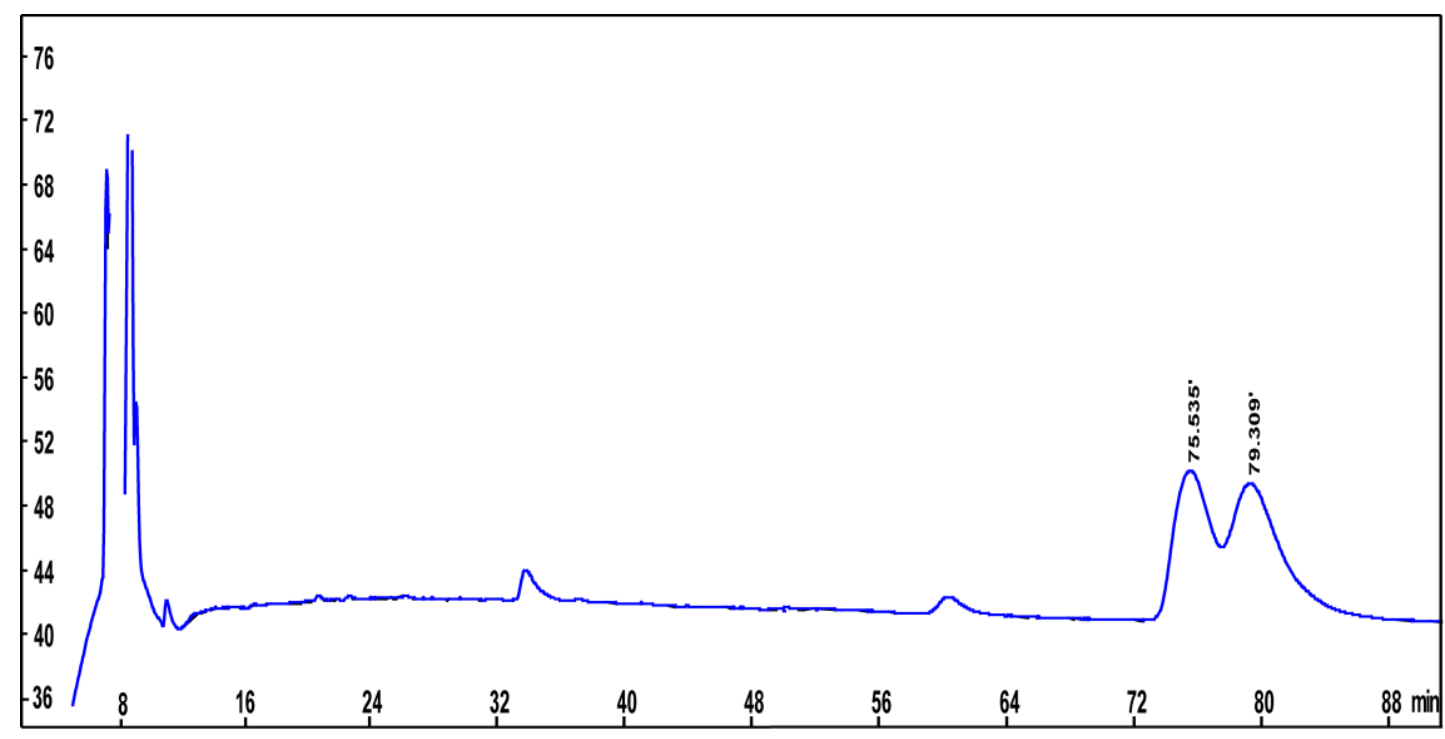

Figure S4. The chiral-phase HPLC spectrum of compound 4 


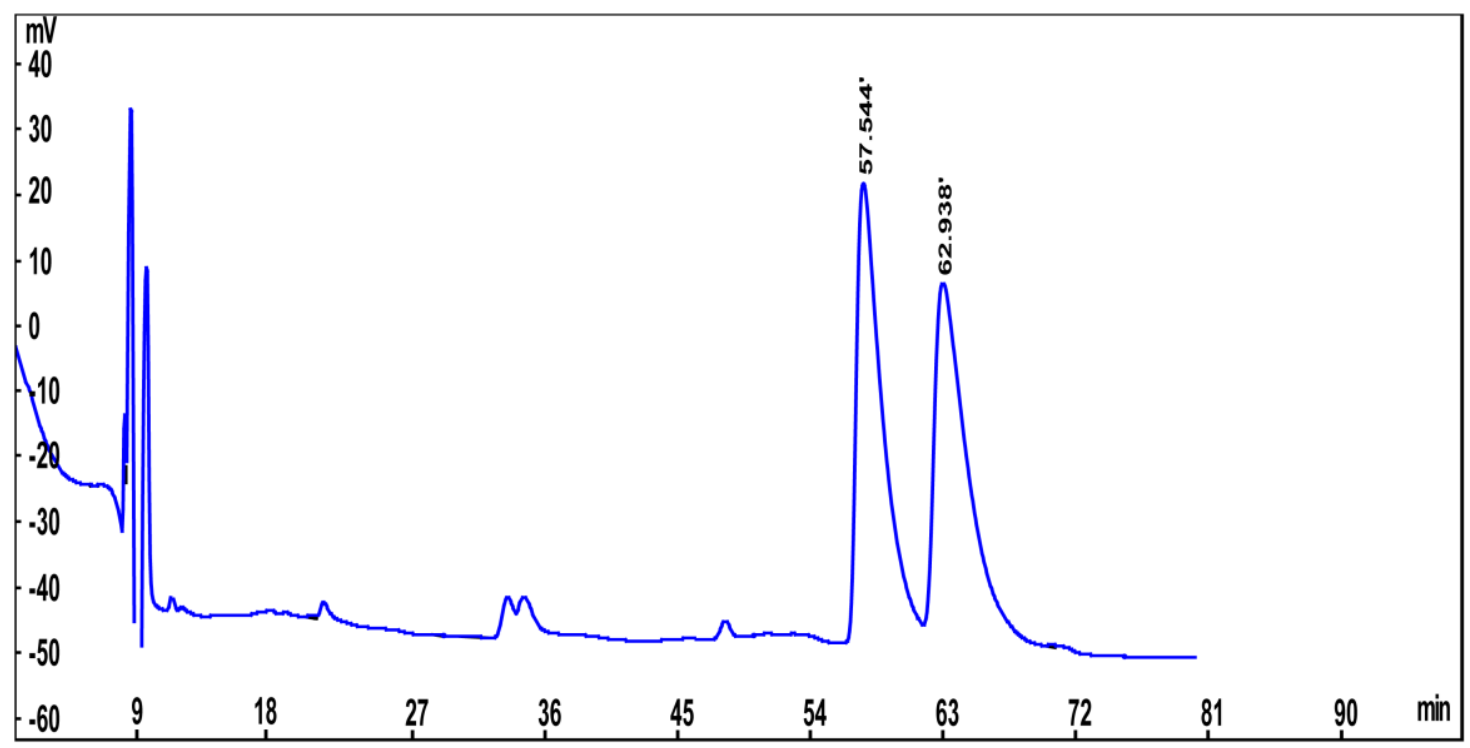

Figure S5. The chiral-phase HPLC spectrum of compound 5

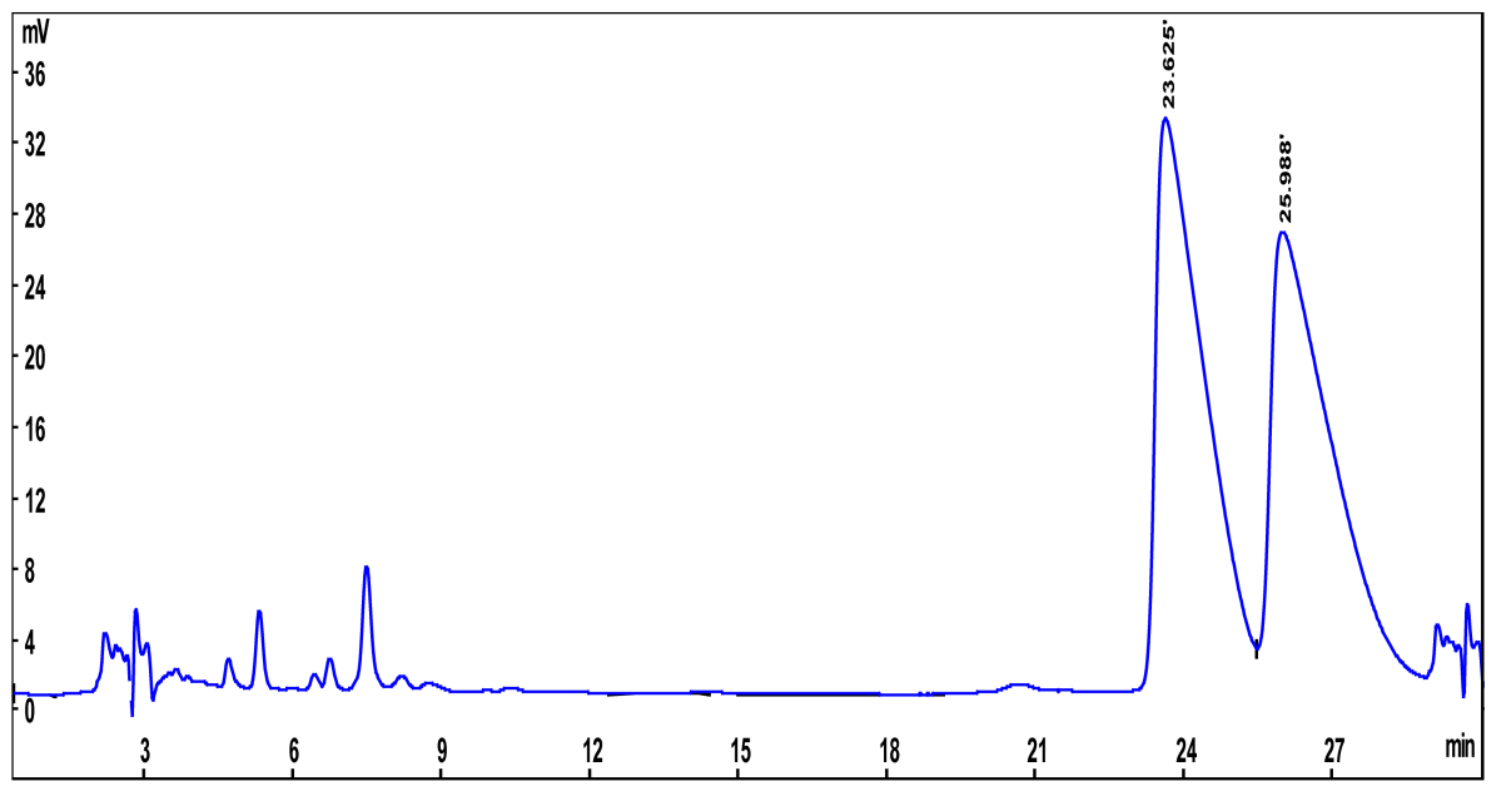

Figure S6. The chiral-phase HPLC spectrum of compound 6 


\section{In silico prediction of ${ }^{13} \mathrm{C}$ NMR chemical shifts and ECD spectrum.}

All calculations were performed using Gaussian $16^{1}$. Conformation search using molecular mechanics calculations was performed in DS (Discovery Studio) 2018 with MMFF94s force field with 20 kcal $\mathrm{mol}^{-1}$ upper energy limit at best level. The conformers performed with the DS 2018 software package were further optimized by using the TDDFT method at the B3LYP/6-31G $(\mathrm{d}, \mathrm{p})$ level, and the frequency was calculated at the same level of theory. For all optimized structures, vibrational spectra were calculated to ensure that no imaginary frequencies for energy minimum were obtained. The average values were obtained by the Boltzmann distributions, using the relative Gibbs free energies as weighting factors. NMR chemical shifts calculations [B3LYP/6-31G+(d,p) level using the polarized continuum model (PCM) model for DMSO] were performed with Gaussian 16. After Boltzmann weighing of the predicted chemical shift of each isomers, the DP4+ parameters were calculated using the excel file provided by Ariel M. Sarotti ${ }^{2}$.

The stable conformers were subjected to ECD calculation by the TDDFT method at the B3LYP/6-311G+ $(\mathrm{d}, \mathrm{p})$ level with the CPCM model in ACN or MeOH. ECD spectra of different conformers were simulated using SpecDis $1.71^{3}$ with a half-bandwidth of $0.3 \mathrm{eV}$, and the final calculated ECD spectra were obtained according to the Boltzmann-calculated contribution of each conformer. The calculated ECD spectra were compared with the experimental data.

Table S1. Cartesian Coordinates, Relative Energies, and Equilibrium Populations of Low-energy Conformers $(>1 \%)$ of $1 R^{*}, 9 S^{*-1}(1 \mathrm{~A})$.

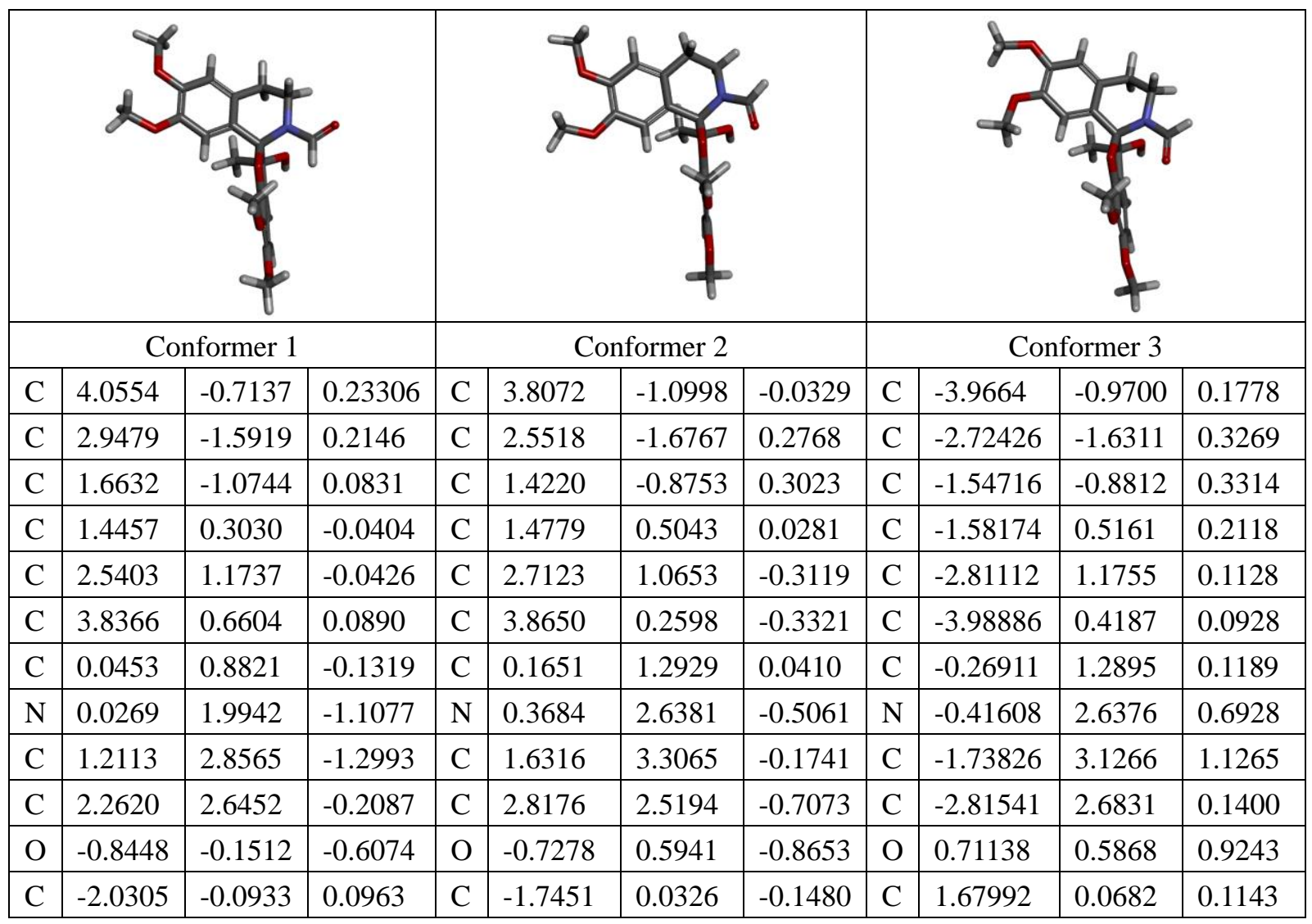




\begin{tabular}{|c|c|c|c|c|c|c|c|c|c|c|c|}
\hline C & 1.9933 & 0.7903 & 1.1632 & $\mathrm{C}$ & -1.7219 & 0.3427 & 1.2095 & $\mathrm{C}$ & 1.53109 & 0.3940 & -1.2305 \\
\hline $\mathrm{C}$ & -0.5952 & 3562 & .2881 & $\mathrm{C}$ & -0.6064 & 1.3280 & 1.4797 & $\mathrm{C}$ & 0.33848 & 1.3093 & -1.4105 \\
\hline $\mathrm{C}$ & 0.1347 & 7945 & 2.5098 & $\mathrm{C}$ & 0.2683 & 1.0029 & 2.6905 & $\mathrm{C}$ & -0.64499 & 0.8335 & -2.4817 \\
\hline $\mathrm{O}$ & -0.5594 & 7687 & & $\mathrm{O}$ & & & & $\mathrm{O}$ & & & -1.8300 \\
\hline $\mathrm{C}$ & -3.1735 & -0.8161 & -0.2652 & $\mathrm{C}$ & -2.7125 & -0.7905 & -0.7210 & $\mathrm{C}$ & & -0.7252 & 0.5891 \\
\hline $\mathrm{C}$ & -4.3267 & 0.6058 & 0.5277 & $\mathrm{C}$ & -3.6959 & -1.3245 & 0.1440 & $\mathrm{C}$ & & -1.1945 & -0.3603 \\
\hline $\mathrm{C}$ & -4.3004 & 0.3059 & 1.5973 & $\mathrm{C}$ & -3.6770 & -1.0303 & 1.5188 & $\mathrm{C}$ & 3.5271 & -0.8666 & -1.7195 \\
\hline $\mathrm{C}$ & -3.1402 & 1.0079 & 1.9230 & $\mathrm{C}$ & -2.6925 & -0.1878 & 2.0501 & $\mathrm{C}$ & 2.46027 & -0.0679 & -2.1531 \\
\hline $\mathrm{O}$ & -3.2485 & -1.5893 & 3957 & $\mathrm{O}$ & -2.6914 & -1.1280 & -2.0477 & $\mathrm{O}$ & 2.788 & -1.0830 & 1.9112 \\
\hline $\mathrm{C}$ & -2.2830 & -2.6421 & -1.5380 & $\mathrm{C}$ & -3.1062 & 821 & -2.9417 & $\mathrm{C}$ & 146 & -0.3183 & 2.6977 \\
\hline $\mathrm{O}$ & -5.5365 & -1.1873 & & $\mathrm{O}$ & -4.6253 & 209 & -0.4560 & $\mathrm{O}$ & & 653 & 0.1520 \\
\hline $\mathrm{C}$ & -5.6055 & -2.5612 & -0.1253 & $\mathrm{C}$ & -5.6361 & 119 & 0.3498 & $\mathrm{C}$ & & & 7391 \\
\hline $\mathrm{O}$ & 5.2826 & -1.2884 & 0.3939 & $\mathrm{O}$ & 4.8818 & -1.9390 & -0.0083 & $\mathrm{O}$ & 242 & .6409 & 0.2277 \\
\hline $\mathrm{C}$ & 6.4266 & -0.4439 & 0.4647 & $\mathrm{C}$ & 6.1702 & -1.4011 & -0.2833 & $\mathrm{C}$ & -5.42664 & -2.5662 & -0.8363 \\
\hline $\mathrm{O}$ & 3.0960 & -2.9457 & 0.4022 & $\mathrm{O}$ & 2.4386 & -2.9996 & 0.6295 & $\mathrm{O}$ & -2.77132 & -2.9874 & 0.4812 \\
\hline $\mathrm{C}$ & 3.7416 & -3.6653 & -0.6572 & $\mathrm{C}$ & 2.6188 & -3.9481 & -0.4318 & $\mathrm{C}$ & -1.55141 & -3.6871 & 0.7167 \\
\hline $\mathrm{C}$ & -1.0272 & 265 & -1.95 & $\mathrm{C}$ & -0.6635 & & -0.9738 & $\mathrm{C}$ & & & 483 \\
\hline $\mathrm{O}$ & -1.0275 & & & $\mathrm{O}$ & & & 750 & $\mathrm{O}$ & & & 138 \\
\hline $\mathrm{H}$ & 0.8332 & & & $\mathrm{H}$ & & & & $\mathrm{H}$ & & & \\
\hline $\mathrm{H}$ & 4.6731 & 1.3501 & & $\mathrm{H}$ & & & -0.5868 & $\mathrm{H}$ & & 058 & 0343 \\
\hline $\mathrm{H}$ & 0.8720 & 3.8952 & -1.3250 & $\mathrm{H}$ & 1.6002 & 4.3119 & -0.6027 & $\mathrm{H}$ & -1.68327 & 4.2163 & 1.1834 \\
\hline $\mathrm{H}$ & 1.6402 & 2.6365 & -2.2845 & $\mathrm{H}$ & 1.7004 & 208 & 157 & $\mathrm{H}$ & 7899 & 398 & 255 \\
\hline $\mathrm{H}$ & 1.9009 & 449 & & $\mathrm{H}$ & 2.8 & 24 & 3027 & $\mathrm{H}$ & 031 & 602 & 656 \\
\hline $\mathrm{H}$ & 3.1709 & & & $\mathrm{H}$ & & & 279 & $\mathrm{H}$ & 7331 & 163 & 3476 \\
\hline $\mathrm{H}$ & & & & $\mathrm{H}$ & & & & $\mathrm{H}$ & & & \\
\hline $\mathrm{H}$ & -0.4093 & & & $\mathrm{H}$ & & & & $\mathrm{H}$ & & & \\
\hline $\mathrm{H}$ & 0.1802 & -0.2960 & 2.4768 & $\mathrm{H}$ & 1.0856 & & 2.7805 & $\mathrm{H}$ & -1.50976 & 1.5008 & -2.5369 \\
\hline $\mathrm{H}$ & -1.0756 & 3.1763 & 0.7359 & $\mathrm{H}$ & -1.8088 & 2.8270 & 1.0928 & $\mathrm{H}$ & & 2.9052 & -1.2531 \\
\hline $\mathrm{H}$ & -5.2168 & 0.4399 & 2.1617 & $\mathrm{H}$ & -4.4302 & -1.4454 & 2.1766 & $\mathrm{H}$ & 4.24872 & -1.2265 & -2.4422 \\
\hline $\mathrm{H}$ & -3.1320 & & & $\mathrm{H}$ & -2.7000 & & 3.1100 & $\mathrm{H}$ & 2.37472 & 927 & -3.2038 \\
\hline $\mathrm{H}$ & -1.2715 & -2.2424 & & $\mathrm{H}$ & & & -2.7796 & $\mathrm{H}$ & & & 2.3385 \\
\hline $\mathrm{H}$ & -2.5619 & -3.1792 & & $\mathrm{H}$ & & & & $\mathrm{H}$ & & & 2.6738 \\
\hline $\mathrm{H}$ & -2.3278 & -3.3236 & -0.6790 & $\mathrm{H}$ & -2.4954 & & -2.8081 & $\mathrm{H}$ & & & 3.7182 \\
\hline $\mathrm{H}$ & -4.8964 & -3.1704 & 0.4480 & $\mathrm{H}$ & -5.2052 & -3.3719 & 1.1131 & $\mathrm{H}$ & 5.18535 & -3.1449 & -1.4907 \\
\hline $\mathrm{H}$ & -6.6236 & -2.8800 & 0.1071 & $\mathrm{H}$ & -6.2496 & -3.3012 & -0.3331 & $\mathrm{H}$ & 6.32531 & -3.0677 & -0.1190 \\
\hline $\mathrm{H}$ & -5.4107 & -2.6757 & -1.1930 & $\mathrm{H}$ & -6.2598 & -1.9503 & 0.8348 & $\mathrm{H}$ & 6.19403 & -1.6852 & -1.2422 \\
\hline $\mathrm{H}$ & 7.2761 & -1.1100 & & $\mathrm{H}$ & 6.2245 & -0.9928 & -1.3003 & $\mathrm{H}$ & -4.70388 & -3.3862 & -0.8392 \\
\hline $\mathrm{H}$ & 6.3535 & 0.2545 & 1.3072 & $\mathrm{H}$ & 6.8656 & -2.2359 & -0.1896 & $\mathrm{H}$ & -5.41149 & -2.0493 & -1.8043 \\
\hline $\mathrm{H}$ & 6.5680 & 0.1175 & -0.4671 & $\mathrm{H}$ & 6.4379 & -0.6211 & 0.4401 & $\mathrm{H}$ & -6.42868 & -2.9561 & -0.6489 \\
\hline $\mathrm{H}$ & 3.7344 & -4.7139 & -0.3543 & $\mathrm{H}$ & 1.8708 & -3.7888 & -1.2183 & $\mathrm{H}$ & -1.8331 & -4.7327 & 0.8458 \\
\hline $\mathrm{H}$ & 4.7728 & -3.3284 & -0.7957 & $\mathrm{H}$ & 2.4710 & -4.9318 & 0.0174 & $\mathrm{H}$ & -1.05523 & -3.3262 & 1.6250 \\
\hline $\mathrm{H}$ & 3.1819 & -3.5517 & -1.5944 & $\mathrm{H}$ & 3.6263 & -3.8835 & -0.8537 & $\mathrm{H}$ & -0.86679 & -3.5953 & -0.1352 \\
\hline $\mathrm{H}$ & -1.8786 & 1.5475 & -1.8256 & $\mathrm{H}$ & -0.3006 & 4.3644 & -1.3832 & $\mathrm{H}$ & 0.39936 & 4.3339 & 1.5100 \\
\hline
\end{tabular}




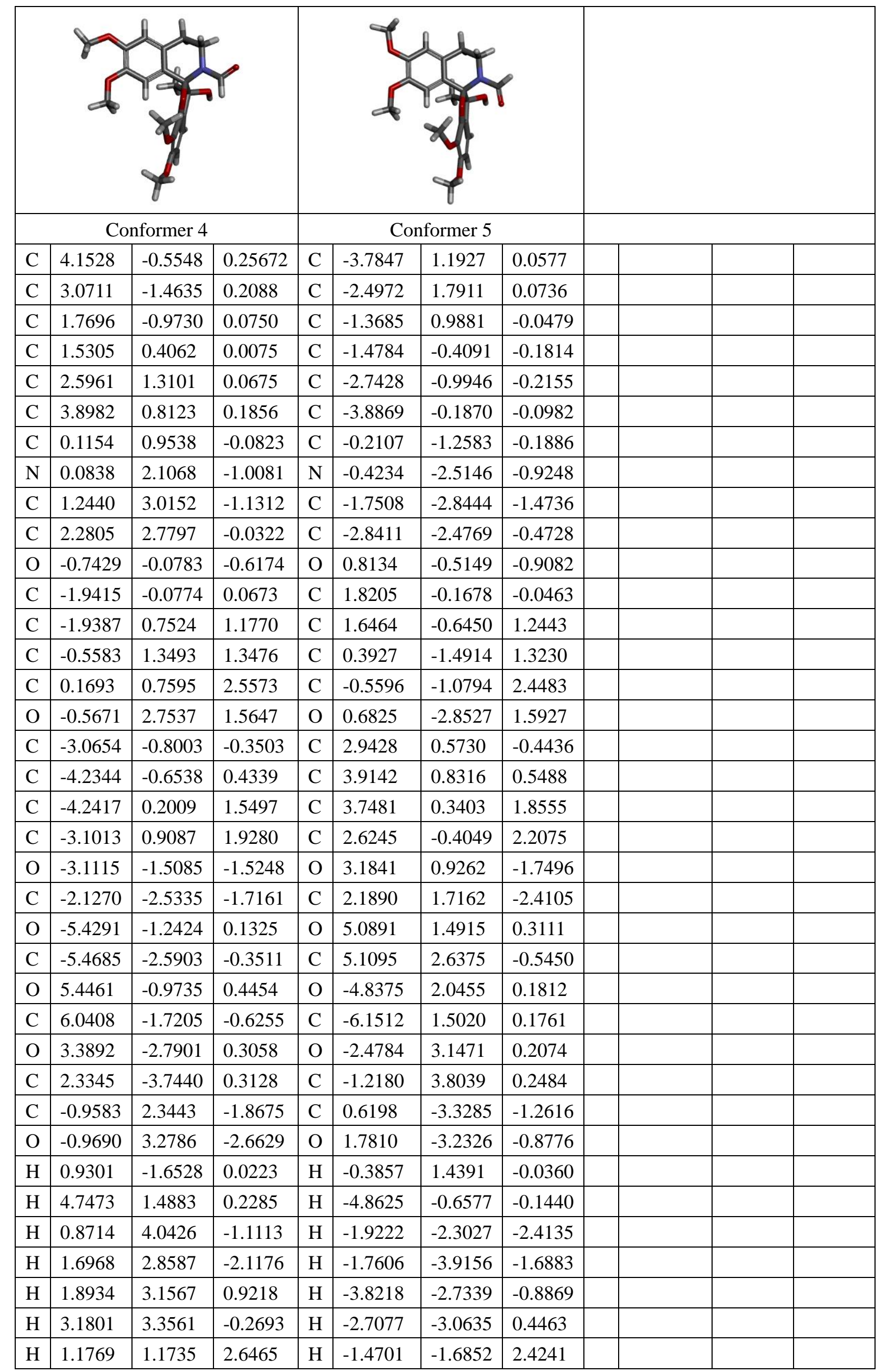




\begin{tabular}{|c|c|c|c|c|c|c|c|c|c|c|}
\hline $\mathrm{H}$ & -0.3946 & 1.0106 & 3.4598 & $\mathrm{H}$ & -0.8329 & -0.0253 & 2.3983 & & & \\
\hline $\mathrm{H}$ & 0.2425 & -0.3275 & 2.4830 & $\mathrm{H}$ & -0.0542 & -1.2689 & 3.3992 & & & \\
\hline $\mathrm{H}$ & -1.0748 & 3.1773 & 0.8577 & $\mathrm{H}$ & 1.3948 & -3.1275 & 0.9874 & & & \\
\hline $\mathrm{H}$ & -5.1690 & 0.2885 & 2.1054 & $\mathrm{H}$ & 4.5292 & 0.5584 & 2.5756 & & & \\
\hline $\mathrm{H}$ & -3.1204 & 1.5674 & 2.7908 & $\mathrm{H}$ & 2.5193 & -0.7965 & 3.2147 & & & \\
\hline $\mathrm{H}$ & -1.1207 & -2.1115 & -1.7777 & $\mathrm{H}$ & 2.0391 & 2.6678 & -1.8822 & & & \\
\hline $\mathrm{H}$ & -2.3809 & -3.0169 & -2.6611 & $\mathrm{H}$ & 1.2391 & 1.1814 & -2.4865 & & & \\
\hline $\mathrm{H}$ & -2.1753 & -3.2694 & -0.9029 & $\mathrm{H}$ & 2.5811 & 1.9155 & -3.4097 & & & \\
\hline $\mathrm{H}$ & -4.7537 & -3.2203 & 0.1920 & $\mathrm{H}$ & 5.1369 & 2.3509 & -1.5980 & & & \\
\hline $\mathrm{H}$ & -6.4826 & -2.9410 & -0.1493 & $\mathrm{H}$ & 4.2386 & 3.2787 & -0.3603 & & & \\
\hline $\mathrm{H}$ & -5.2615 & -2.6364 & -1.4217 & $\mathrm{H}$ & 6.0185 & 3.1823 & -0.2808 & & & \\
\hline $\mathrm{H}$ & 7.0672 & -1.9218 & -0.3139 & $\mathrm{H}$ & -6.2960 & 0.7979 & 1.0053 & & & \\
\hline $\mathrm{H}$ & 6.0492 & -1.1256 & -1.5477 & $\mathrm{H}$ & -6.8226 & 2.3523 & 0.3007 & & & \\
\hline $\mathrm{H}$ & 5.5131 & -2.6631 & -0.7939 & $\mathrm{H}$ & -6.3729 & 0.9995 & -0.7741 & & & \\
\hline $\mathrm{H}$ & 2.8158 & -4.7165 & 0.4226 & $\mathrm{H}$ & -1.4425 & 4.8642 & 0.3699 & & & \\
\hline $\mathrm{H}$ & 1.7678 & -3.7220 & -0.6266 & $\mathrm{H}$ & -0.6130 & 3.4591 & 1.0963 & & & \\
\hline $\mathrm{H}$ & 1.6526 & -3.5786 & 1.1561 & $\mathrm{H}$ & -0.6585 & 3.6545 & -0.6838 & & & \\
\hline $\mathrm{H}$ & -1.7900 & 1.6340 & -1.7913 & $\mathrm{H}$ & 0.3106 & -4.1379 & -1.9457 & & & \\
\hline
\end{tabular}

Table S2. Cartesian Coordinates, Relative Energies, and Equilibrium Populations of Low-energy Conformers $(>1 \%)$ of $1 S *, 9 S *-1(1 \mathrm{~B})$.

\begin{tabular}{|l|l|l|l|l|l|l|l|l|l|l|l|}
\hline \multicolumn{3}{|c|}{ Conformer 1} & \multicolumn{3}{|c|}{ Conformer 2} & \multicolumn{3}{c|}{ Conformer 3} \\
\hline $\mathrm{C}$ & 3.1720 & -2.1480 & 1.014 & $\mathrm{C}$ & 3.2670 & -2.1700 & 1.0000 & $\mathrm{C}$ & 3.7720 & -1.903 & 0.8730 \\
\hline $\mathrm{C}$ & 1.7990 & -2.3690 & 0.7430 & $\mathrm{C}$ & 1.8620 & -2.4340 & 1.0040 & $\mathrm{C}$ & 2.4180 & -2.3270 & 1.0460 \\
\hline $\mathrm{C}$ & 0.9770 & -1.2770 & 0.3590 & $\mathrm{C}$ & 0.9600 & -1.4100 & 0.6300 & $\mathrm{C}$ & 1.3560 & -1.4230 & 0.7330 \\
\hline $\mathrm{C}$ & 1.4800 & 0.0480 & 0.2810 & $\mathrm{C}$ & 1.4150 & -0.1170 & 0.2640 & $\mathrm{C}$ & 1.6180 & -0.1080 & 0.2590 \\
\hline $\mathrm{C}$ & 2.8510 & 0.2690 & 0.6190 & $\mathrm{C}$ & 2.8080 & 0.1380 & 0.2610 & $\mathrm{C}$ & 2.9710 & 0.2910 & 0.1000 \\
\hline $\mathrm{C}$ & 3.6870 & -0.8250 & 0.9560 & $\mathrm{C}$ & 3.7250 & -0.8770 & 0.6200 & $\mathrm{C}$ & 4.0280 & -0.5980 & 0.3980 \\
\hline $\mathrm{C}$ & 0.5520 & 1.1700 & -0.1490 & $\mathrm{C}$ & 0.4500 & 0.9800 & -0.1230 & $\mathrm{C}$ & 0.5090 & 0.8690 & -0.0840 \\
\hline $\mathrm{N}$ & 1.1370 & 2.5030 & 0.1600 & $\mathrm{~N}$ & 0.9180 & 2.3100 & 0.3850 & $\mathrm{~N}$ & 0.8930 & 2.2520 & 0.3790 \\
\hline $\mathrm{C}$ & 2.5850 & 2.6840 & -0.1160 & $\mathrm{C}$ & 2.3820 & 2.5830 & 0.5200 & $\mathrm{C}$ & 2.3120 & 2.7020 & 0.2520 \\
\hline $\mathrm{C}$ & 3.4190 & 1.6420 & 0.6450 & $\mathrm{C}$ & 3.2640 & 1.4910 & -0.1210 & $\mathrm{C}$ & 3.2520 & 1.6580 & -0.3830 \\
\hline $\mathrm{O}$ & -0.6400 & 1.0190 & 0.6570 & $\mathrm{O}$ & -0.7920 & 0.7240 & 0.5700 & $\mathrm{O}$ & -0.6910 & 0.5090 & 0.6450 \\
\hline $\mathrm{C}$ & -1.7080 & 0.6870 & -0.0720 & $\mathrm{C}$ & -1.8380 & 0.6760 & -0.2570 & $\mathrm{C}$ & -1.7860 & 0.5600 & -0.1230 \\
\hline $\mathrm{C}$ & -1.4300 & 0.6950 & -1.4450 & $\mathrm{C}$ & -1.4600 & 0.8640 & -1.5910 & $\mathrm{C}$ & -1.4620 & 0.7540 & -1.4720 \\
\hline $\mathrm{C}$ & 0.0240 & 1.0770 & -1.6660 & $\mathrm{C}$ & 0.0500 & 1.0140 & -1.6780 & $\mathrm{C}$ & 0.0460 & 0.8510 & -1.6230 \\
\hline
\end{tabular}




\begin{tabular}{|c|c|c|c|c|c|c|c|c|c|c|c|}
\hline C & 0.0820 & 2.3780 & -2.5010 & $\mathrm{C}$ & 0.4360 & 2.2910 & -2.4580 & $\mathrm{C}$ & 0.4130 & 2.0780 & -2.4900 \\
\hline $\mathrm{O}$ & 0.6960 & 0.0660 & -2.4180 & $\mathrm{O}$ & 0.5830 & -0.1000 & -2.3960 & $\mathrm{O}$ & 0.5070 & -0.3160 & -2.3040 \\
\hline C & -3.0160 & 0.3360 & 0.3880 & $\mathrm{C}$ & -3.2120 & 0.4660 & 0.0840 & $\mathrm{C}$ & -3.1610 & 0.4860 & 0.2720 \\
\hline $\mathrm{C}$ & -4.0390 & 0.0390 & -0.5540 & $\mathrm{C}$ & -4.2040 & 0.5150 & -0.9510 & 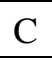 & -4.1860 & 0.6200 & -0.7060 \\
\hline C & -3.7500 & 0.0660 & -1.9390 & $\mathrm{C}$ & -3.8040 & 0.7290 & -2.2850 & C & -3.8430 & 0.7800 & -2.0690 \\
\hline C & -2.4530 & 0.3850 & -2.3720 & $\mathrm{C}$ & -2.4470 & 0.8930 & -2.6020 & C & -2.4900 & 0.8400 & -2.4410 \\
\hline $\mathrm{O}$ & -3.4380 & 0.2290 & 1.6650 & $\mathrm{O}$ & -3.6920 & 0.2190 & 1.3200 & $\mathrm{O}$ & -3.6610 & 0.3340 & 1.5160 \\
\hline $\mathrm{C}$ & -2.5220 & 0.3670 & 2.7530 & $\mathrm{C}$ & -2.8150 & 0.0160 & 2.4310 & $\mathrm{C}$ & -2.7970 & 0.2400 & 2.6480 \\
\hline $\mathrm{O}$ & -5.2670 & -0.2710 & -0.0750 & $\mathrm{O}$ & -5.5420 & 0.3730 & -0.7830 & $\mathrm{O}$ & -5.4720 & 0.6050 & -0.2800 \\
\hline $\mathrm{C}$ & -6.3760 & -0.5520 & -0.9360 & $\mathrm{C}$ & -6.1630 & 0.1160 & 0.4780 & & -6.5720 & 0.9130 & -1.1460 \\
\hline $\mathrm{O}$ & 3.9340 & -3.2210 & 1.3280 & $\mathrm{O}$ & 4.1240 & -3.1500 & 1.3620 & & 4.8720 & -2.6520 & 1.1320 \\
\hline $\mathrm{C}$ & 5.3410 & -3.1140 & 1.5780 & $\mathrm{C}$ & 5.5420 & -2.9600 & 1.3970 & & 4.8140 & -3.9880 & 1.6360 \\
\hline $\mathrm{O}$ & 1.3180 & -3.6270 & 0.8640 & $\mathrm{O}$ & 1.2900 & -3.6130 & 1.3460 & $\mathrm{O}$ & 2.1880 & -3.5790 & 1.5040 \\
\hline $\mathrm{C}$ & -0.0700 & -3.9290 & 0.6790 & $\mathrm{C}$ & 2.0410 & -4.7700 & 1.7250 & $\mathrm{C}$ & 0.8700 & -4.0880 & 1.7180 \\
\hline $\mathrm{C}$ & 0.3780 & 3.5370 & 0.6700 & $\mathrm{C}$ & 0.0270 & 3.3050 & 0.7350 & $c$ & 0.0220 & 3.1720 & 0.9340 \\
\hline $\mathrm{O}$ & 0.8680 & 4.6150 & 0.9160 & $\mathrm{O}$ & 0.4150 & 4.3740 & 1.1480 & 8 & -1.1510 & 2.9460 & 1.1100 \\
\hline $\mathrm{H}$ & -0.0590 & -1.4550 & 0.1200 & $\mathrm{H}$ & -0.0980 & -1.6280 & 0.6270 & 11 & 0.3290 & -1.7340 & 0.8540 \\
\hline $\mathrm{H}$ & 4.7270 & -0.6350 & & $\mathrm{H}$ & & -0.6490 & 0.6010 & $\mathrm{H}$ & & -0.2750 & 0.2630 \\
\hline $\mathrm{H}$ & & & & $\mathrm{H}$ & & & & & & & \\
\hline $\mathrm{H}$ & 2.7490 & 2.5670 & -1.1870 & $\mathrm{H}$ & 2.6160 & 3.5450 & 0.0610 & 1 & 2.6780 & 2.9480 & 1.2510 \\
\hline $\mathrm{H}$ & 3.5040 & 1.9470 & 1.6900 & $\mathrm{H}$ & 3.2290 & 1.5700 & -1.2080 & $\mathrm{H}$ & 3.1600 & 1.6590 & -1.4670 \\
\hline $\mathrm{H}$ & 4.4340 & 1.6370 & 0.2440 & $\mathrm{H}$ & 4.3050 & 1.6510 & 0.1650 & $\mathrm{H}$ & 4.2850 & 1.9410 & -0.1780 \\
\hline $\mathrm{H}$ & 1.1070 & 2.7030 & -2.6730 & $\mathrm{H}$ & 0.1270 & 2.2440 & -3.5030 & $\mathrm{H}$ & 0.0330 & 3.0070 & -2.0640 \\
\hline $\mathrm{H}$ & & & & $\mathrm{H}$ & & & & $\mathrm{H}$ & & 2.1920 & -2.6090 \\
\hline $\mathrm{H}$ & -0.4440 & 3.1990 & -2.0110 & $\mathrm{H}$ & 1.5150 & 2.4510 & -2.4490 & $\mathrm{H}$ & -0.0020 & 2.0040 & -3.4960 \\
\hline $\mathrm{H}$ & 0.3160 & 0.0110 & -3.3110 & $\mathrm{H}$ & 0.2850 & -0.0710 & -3.3200 & $\mathrm{H}$ & 0.1730 & -0.3210 & -3.2160 \\
\hline $\mathrm{H}$ & -4.5060 & -0.1560 & -2.6760 & $\mathrm{H}$ & -4.5420 & 0.7660 & -3.0740 & $\mathrm{H}$ & -4.5990 & 0.8600 & -2.8350 \\
\hline $\mathrm{H}$ & -2.2330 & 0.3970 & -3.4290 & $\mathrm{H}$ & -2.1530 & 1.0440 & -3.6300 & $\mathrm{H}$ & -2.2310 & 0.9610 & -3.4830 \\
\hline $\mathrm{H}$ & -2.0900 & 1.3690 & 2.7550 & $\mathrm{H}$ & -3.4140 & -0.2060 & 3.3140 & $\mathrm{H}$ & -2.1410 & -0.6280 & 2.5510 \\
\hline $\mathrm{H}$ & -3.0620 & 0.2180 & & $\mathrm{H}$ & -2.1490 & -0.8260 & & $\mathrm{H}$ & -2.2020 & 1.1500 & 2.7460 \\
\hline $\mathrm{H}$ & -1.7330 & -0.3830 & 2.6780 & $\mathrm{H}$ & -2.2360 & 0.9210 & 2.6220 & $\mathrm{H}$ & -3.4050 & 0.1210 & 3.5450 \\
\hline $\mathrm{H}$ & -7.2510 & -0.7630 & -0.3210 & $\mathrm{H}$ & -7.2390 & 0.0400 & 0.3240 & $\mathrm{H}$ & -6.4560 & 1.9160 & -1.5600 \\
\hline $\mathrm{H}$ & -6.1630 & -1.4280 & -1.5510 & $\mathrm{H}$ & -5.9750 & 0.9390 & 1.1680 & $\mathrm{H}$ & -7.4950 & 0.8780 & -0.5670 \\
\hline $\mathrm{H}$ & -6.5910 & 0.3110 & -1.5680 & $\mathrm{H}$ & -5.8100 & -0.8310 & 0.8890 & $\mathrm{H}$ & -6.6350 & 0.1780 & -1.9490 \\
\hline $\mathrm{H}$ & 5.5220 & -2.4700 & 2.4410 & $\mathrm{H}$ & 5.9140 & -2.7070 & 0.4030 & $\mathrm{H}$ & 4.2980 & -4.6380 & 0.9270 \\
\hline $\mathrm{H}$ & 5.7350 & -4.1070 & 1.7950 & $\mathrm{H}$ & 6.0110 & -3.8890 & 1.7200 & $\mathrm{H}$ & 4.3180 & -4.0040 & 2.6080 \\
\hline $\mathrm{H}$ & 5.8510 & -2.7190 & 0.6980 & $\mathrm{H}$ & 5.7980 & -2.1730 & 2.1100 & $\mathrm{H}$ & 5.8310 & -4.3560 & 1.7640 \\
\hline $\mathrm{H}$ & -0.3740 & -3.6940 & -0.3420 & $\mathrm{H}$ & 2.6160 & -4.5690 & 2.6300 & $\mathrm{H}$ & 0.9460 & -5.1090 & 2.0930 \\
\hline $\mathrm{H}$ & -0.2210 & -4.9950 & 0.8500 & $\mathrm{H}$ & 2.6960 & -5.0790 & 0.9090 & $\mathrm{H}$ & 0.3150 & -4.1010 & 0.7780 \\
\hline $\mathrm{H}$ & -0.6770 & -3.3710 & 1.3940 & $\mathrm{H}$ & 1.3450 & -5.5820 & 1.9330 & $\mathrm{H}$ & 0.3460 & -3.4820 & 2.4600 \\
\hline $\mathrm{H}$ & -0.6760 & 3.3930 & 0.8560 & $\mathrm{H}$ & -1.0360 & 3.1440 & 0.6420 & $\mathrm{H}$ & 0.4040 & 4.1400 & 1.2280 \\
\hline
\end{tabular}


Table S3. Cartesian Coordinates, Relative Energies, and Equilibrium Populations of Low-energy

Conformers $(>1 \%)$ of $9 R^{*}-2$.

\begin{tabular}{|c|c|c|c|c|c|c|c|}
\hline \multicolumn{4}{|c|}{ Conformer 1} & \multicolumn{4}{|c|}{ Conformer 2} \\
\hline C & -1.3676 & 4.0906 & -0.5981 & C & -1.3273 & 4.1193 & -0.5538 \\
\hline $\mathrm{C}$ & -1.3982 & 2.7555 & -1.0747 & $\mathrm{C}$ & -1.3919 & 2.7836 & -1.0405 \\
\hline $\mathrm{C}$ & -0.3867 & 1.8656 & -0.6508 & $\mathrm{C}$ & -0.3796 & 1.8669 & -0.6446 \\
\hline $\mathrm{C}$ & 0.6766 & 2.2578 & 0.1735 & $\mathrm{C}$ & 0.7106 & 2.2302 & 0.1584 \\
\hline $\mathrm{C}$ & 0.6630 & 3.5942 & 0.6752 & $\mathrm{C}$ & 0.7463 & 3.5625 & 0.6520 \\
\hline $\mathrm{C}$ & -0.3889 & 4.4754 & 0.3296 & $\mathrm{C}$ & -0.2688 & 4.4715 & 0.2995 \\
\hline $\mathrm{C}$ & 1.7492 & 1.3779 & 0.5262 & $\mathrm{C}$ & 1.7757 & 1.3433 & 0.5209 \\
\hline $\mathrm{N}$ & 2.6919 & 1.8871 & 1.3687 & $\mathrm{~N}$ & 2.7201 & 1.8353 & 1.3729 \\
\hline $\mathrm{C}$ & 2.7193 & 3.1455 & 1.8627 & $\mathrm{C}$ & 2.7675 & 3.0890 & 1.8735 \\
\hline $\mathrm{C}$ & 1.7060 & 4.0429 & 1.5032 & $\mathrm{C}$ & 1.7728 & 3.9972 & 1.5025 \\
\hline $\mathrm{C}$ & 1.9238 & -0.0732 & 0.1109 & $\mathrm{C}$ & 1.9378 & -0.1016 & 0.1054 \\
\hline $\mathrm{C}$ & 0.9808 & -0.9656 & 0.8111 & $\mathrm{C}$ & 9892 & -0.9822 & 0.8100 \\
\hline $\mathrm{C}$ & 0.7342 & -1.0136 & & $\mathrm{C}$ & & -1.0210 & 2.1836 \\
\hline $\mathrm{C}$ & -0.2032 & -1.9316 & 2.6703 & $\mathrm{C}$ & -0.2045 & -1.9329 & 2.6707 \\
\hline $\mathrm{C}$ & -0.9133 & -2.7882 & 1.7955 & $\mathrm{C}$ & -0.9161 & -2.7895 & 1.7969 \\
\hline $\mathrm{C}$ & -0.6496 & -2.7468 & 0.3963 & $\mathrm{C}$ & -0.6522 & -2.7507 & 0.3975 \\
\hline $\mathrm{C}$ & 0.3208 & -1.8220 & -0.0766 & C & 0.3217 & -1.8321 & -0.0769 \\
\hline $\mathrm{O}$ & -2.0757 & 5.1407 & -1.1031 & $\mathrm{O}$ & .2098 & 5.0922 & -0.9574 \\
\hline $\mathrm{O}$ & -2.3338 & 2.3477 & & $\mathrm{O}$ & & 2.3961 & -1.9477 \\
\hline $\mathrm{C}$ & -2.7545 & 5.2101 & -2.3540 & $\mathrm{C}$ & -2.1286 & 6.5269 & -0.7771 \\
\hline $\mathrm{C}$ & -2.3166 & 1.1628 & -2.7919 & $\mathrm{C}$ & -2.3321 & 1.2072 & -2.7707 \\
\hline $\mathrm{O}$ & -1.8642 & -3.6229 & 2.3122 & $\mathrm{O}$ & -1.8675 & -3.6236 & 2.3131 \\
\hline $\mathrm{C}$ & -2.4767 & -3.6718 & 3.6091 & $\mathrm{C}$ & -2.4787 & -3.6734 & 3.6103 \\
\hline $\mathrm{O}$ & -1.3373 & -3.4347 & -0.5637 & $\mathrm{O}$ & -1.3408 & -3.4367 & -0.5626 \\
\hline $\mathrm{C}$ & -2.5921 & -4.1177 & -0.4555 & $\mathrm{C}$ & -2.5949 & -4.1198 & -0.4561 \\
\hline $\mathrm{C}$ & 0.7411 & -1.4579 & -1.3410 & $\mathrm{C}$ & 0.7391 & -1.4683 & -1.3432 \\
\hline $\mathrm{O}$ & 1.6122 & -0.4191 & -1.2566 & $\mathrm{O}$ & 1.6183 & -0.4372 & -1.2609 \\
\hline $\mathrm{O}$ & 0.3773 & -1.9550 & -2.441 & $\mathrm{O}$ & 0.3677 & -1.9584 & -2.4431 \\
\hline $\mathrm{C}$ & 3.3639 & -0.6196 & 0.3375 & $\mathrm{C}$ & 3.3778 & -0.6470 & 0.3311 \\
\hline $\mathrm{H}$ & -0.4333 & 0.9116 & -0.9667 & $\mathrm{H}$ & -0.4508 & 0.9119 & -0.9595 \\
\hline $\mathrm{H}$ & -0.3964 & 5.4355 & 0.6886 & $\mathrm{H}$ & -0.2082 & 5.4076 & 0.6868 \\
\hline $\mathrm{H}$ & 3.4747 & 3.4328 & 2.4842 & $\mathrm{H}$ & 3.5165 & 3.3692 & 2.5047 \\
\hline $\mathrm{H}$ & 1.7439 & 5.0085 & 1.8417 & $\mathrm{H}$ & 1.8104 & 4.9580 & 1.8564 \\
\hline
\end{tabular}




\begin{tabular}{|c|c|c|c|c|c|c|c|}
\hline $\mathrm{H}$ & 1.2182 & -0.3882 & 2.8314 & $\mathrm{H}$ & 1.2217 & -0.3933 & 2.8287 \\
\hline $\mathrm{H}$ & -0.3472 & -1.9620 & 3.6739 & $\mathrm{H}$ & -0.3502 & -1.9600 & 3.6745 \\
\hline $\mathrm{H}$ & -3.0927 & 6.2329 & -2.5125 & $\mathrm{H}$ & -3.1137 & 6.9789 & -0.8638 \\
\hline $\mathrm{H}$ & -3.6045 & 4.5310 & -2.4144 & $\mathrm{H}$ & -1.4831 & 6.9324 & -1.5590 \\
\hline $\mathrm{H}$ & -2.0275 & 4.9629 & -3.1308 & $\mathrm{H}$ & -1.7284 & 6.8349 & 0.1894 \\
\hline $\mathrm{H}$ & -1.505 & 1.2812 & -3.5123 & $\mathrm{H}$ & -1.5175 & 1.3105 & -3.4912 \\
\hline $\mathrm{H}$ & -3.2558 & 1.0588 & -3.3298 & $\mathrm{H}$ & -3.2679 & 1.1065 & -3.3158 \\
\hline $\mathrm{H}$ & -2.1529 & 0.2523 & -2.2126 & $\mathrm{H}$ & -2.1802 & 0.2895 & -2.1982 \\
\hline $\mathrm{H}$ & -2.8839 & -4.6648 & 3.7888 & $\mathrm{H}$ & -2.8871 & -4.6664 & 3.7878 \\
\hline $\mathrm{H}$ & -3.2928 & -2.9481 & 3.6114 & $\mathrm{H}$ & -3.2938 & -2.9485 & 3.6148 \\
\hline $\mathrm{H}$ & -1.7937 & -3.4383 & 4.4249 & $\mathrm{H}$ & -1.7942 & -3.4426 & 4.4256 \\
\hline $\mathrm{H}$ & -2.6265 & -4.8206 & $0.3750 \mathrm{c}$ & $\mathrm{H}$ & -2.6274 & -4.8248 & 0.3728 \\
\hline $\mathrm{H}$ & -2.8025 & -4.6677 & -1.3715 & $\mathrm{H}$ & -2.8033 & -4.6674 & -1.3739 \\
\hline $\mathrm{H}$ & -3.3615 & -3.3554 & -0.3207 & $\mathrm{H}$ & -3.3652 & -3.3588 & -0.3196 \\
\hline $\mathrm{H}$ & 3.4474 & -1.6554 & -0.0026 & $\mathrm{H}$ & 3.4622 & -1.6811 & -0.0103 \\
\hline $\mathrm{H}$ & 3.6323 & -0.5888 & 1.3958 & $\mathrm{H}$ & 3.6453 & -0.6157 & 1.3900 \\
\hline $\mathrm{H}$ & 4.0746 & -0.0168 & -0.2284 & $\mathrm{H}$ & 4.0866 & -0.0393 & -0.2327 \\
\hline \multicolumn{4}{|c|}{ Conformer 3} & \multicolumn{4}{|c|}{ Conformer 4} \\
\hline $\mathrm{C}$ & -1.2997 & 4.1729 & -0.5603 & $\mathrm{C}$ & -1.3107 & 4.1322 & -0.5509 \\
\hline $\mathrm{C}$ & -1.4281 & 2.8371 & -1.0316 & $\mathrm{C}$ & -1.3633 & 2.7954 & -1.0302 \\
\hline $\mathrm{C}$ & -0.4715 & 1.8653 & -0.6401 & $\mathrm{C}$ & -0.3676 & 1.8804 & -0.6085 \\
\hline $\mathrm{C}$ & 0.6560 & 2.2030 & 0.1522 & $\mathrm{C}$ & 0.7185 & 2.2430 & 0.2212 \\
\hline $\mathrm{C}$ & 0.7339 & 3.5395 & 0.6413 & $\mathrm{C}$ & 0.7493 & 3.5861 & 0.6855 \\
\hline $\mathrm{C}$ & -0.2346 & 4.4849 & 0.2894 & $\mathrm{C}$ & -0.2571 & 4.4909 & 0.3142 \\
\hline $\mathrm{C}$ & 1.7318 & 1.3263 & 0.5050 & $\mathrm{C}$ & 1.7771 & 1.3691 & 0.6254 \\
\hline $\mathrm{N}$ & 2.6889 & 1.8111 & 1.3516 & $\mathrm{~N}$ & 2.7300 & 1.8696 & 1.4596 \\
\hline $\mathrm{C}$ & 2.7525 & 3.0591 & 1.8575 & $\mathrm{C}$ & 2.7789 & 3.1353 & 1.9187 \\
\hline $\mathrm{C}$ & 1.7656 & 3.9687 & 1.4931 & $\mathrm{C}$ & 1.7841 & 4.0332 & 1.5305 \\
\hline $\mathrm{C}$ & 1.9172 & -0.1101 & 0.0932 & $\mathrm{C}$ & 1.9732 & -0.0652 & 0.2307 \\
\hline $\mathrm{C}$ & 0.9780 & -0.9903 & 0.8027 & $\mathrm{C}$ & 0.9890 & -0.9821 & 0.8391 \\
\hline $\mathrm{C}$ & 0.7334 & -1.0278 & 2.1779 & $\mathrm{C}$ & 0.7240 & -1.1548 & 2.2053 \\
\hline $\mathrm{C}$ & -0.2051 & -1.9408 & 2.6667 & $\mathrm{C}$ & -0.2115 & -2.1262 & 2.5998 \\
\hline $\mathrm{C}$ & -0.9160 & -2.7988 & 1.7940 & $\mathrm{C}$ & -0.9449 & -2.8362 & 1.6288 \\
\hline $\mathrm{C}$ & -0.6526 & -2.7633 & 0.3940 & $\mathrm{C}$ & -0.6580 & -2.6810 & 0.2452 \\
\hline $\mathrm{C}$ & 0.3136 & -1.8406 & -0.0838 & $\mathrm{C}$ & 0.3334 & -1.7260 & -0.1508 \\
\hline $\mathrm{O}$ & -2.1583 & 5.1759 & -0.9153 & $\mathrm{O}$ & -2.1983 & 5.0903 & -0.9667 \\
\hline $\mathrm{O}$ & -2.3519 & 2.4231 & -1.9472 & $\mathrm{O}$ & -2.3041 & 2.4163 & -1.9525 \\
\hline C & -2.0682 & 6.6003 & -0.7319 & C & -2.1477 & 6.5256 & -0.7887 \\
\hline
\end{tabular}




\begin{tabular}{|l|l|l|l|l|l|l|l|}
\hline C & -3.1732 & 3.2323 & -2.7985 & C & -2.3322 & 1.2234 & -2.7661 \\
\hline O & -1.8669 & -3.6300 & 2.3133 & O & -2.0212 & -3.4873 & 2.1649 \\
\hline C & -2.4777 & -3.6705 & 3.6106 & C & -3.1973 & -3.9597 & 1.5116 \\
\hline O & -1.3370 & -3.4525 & -0.5657 & O & -1.3954 & -3.4153 & -0.6306 \\
\hline C & -2.5965 & -4.1219 & -0.4541 & C & -1.5076 & -3.3540 & -2.0471 \\
\hline C & 0.7249 & -1.4724 & -1.3517 & C & 0.8513 & -1.2738 & -1.3574 \\
\hline O & 1.5967 & -0.4366 & -1.2716 & O & 1.7385 & -0.2828 & -1.1548 \\
\hline O & 0.3650 & -1.9694 & -2.4514 & O & 0.6399 & -1.5782 & -2.5607 \\
\hline C & 3.3619 & -0.6485 & 0.3233 & C & 3.4104 & -0.6290 & 0.4272 \\
\hline H & -0.5885 & 0.9181 & -0.9994 & H & -0.4471 & 0.9265 & -0.9196 \\
\hline H & -0.1520 & 5.4190 & 0.6768 & H & -0.2062 & 5.4339 & 0.6876 \\
\hline H & 3.5069 & 3.3361 & 2.4853 & H & 3.5369 & 3.4267 & 2.5348 \\
\hline H & 1.8106 & 4.9291 & 1.8479 & H & 1.8220 & 4.9994 & 1.8639 \\
\hline H & 1.2169 & -0.3990 & 2.8226 & H & 1.1767 & -0.5674 & 2.9095 \\
\hline H & -0.3481 & -1.9667 & 3.6707 & H & -0.4186 & -2.2471 & 3.5942 \\
\hline H & -3.0578 & 7.0440 & -0.8027 & H & -3.1391 & 6.9577 & -0.9041 \\
\hline H & -1.4385 & 6.9939 & -1.5307 & H & -1.4904 & 6.9421 & -1.5544 \\
\hline H & -1.6477 & 6.9000 & 0.2281 & H & -1.7778 & 6.8362 & 0.1892 \\
\hline H & -3.7453 & 3.9781 & -2.2490 & H & -1.5199 & 1.2920 & -3.4930 \\
\hline H & -3.8741 & 2.6111 & -3.3523 & H & -3.2744 & 1.1484 & -3.3071 \\
\hline H & -2.5085 & 3.7307 & -3.5069 & H & -2.2073 & 0.3095 & -2.1828 \\
\hline H & -2.8828 & -4.6629 & 3.7966 & H & -3.9037 & -4.3111 & 2.2622 \\
\hline H & -3.2940 & -2.9471 & 3.6075 & H & -2.9880 & -4.7657 & 0.8085 \\
\hline H & -1.7949 & -3.4309 & 4.4246 & H & -3.6447 & -3.1144 & 0.9845 \\
\hline H & -2.6365 & -4.8186 & 0.3810 & H & -1.8364 & -2.3461 & -2.3101 \\
\hline H & -2.8110 & -4.6753 & -1.3666 & H & -2.2482 & -4.0656 & -2.3996 \\
\hline H & -3.3567 & -3.3495 & -0.3242 & H & -0.5592 & -3.5968 & -2.5215 \\
\hline H & 3.4530 & -1.6823 & -0.0161 & H & 3.4760 & -1.6594 & 0.0651 \\
\hline H & 3.6291 & -0.6155 & 1.3830 & H & 3.6979 & -0.6163 & 1.4787 \\
\hline H & 4.0699 & -0.0374 & -0.2380 & H & 4.1162 & -0.0254 & -0.1469 \\
\hline & & & & & & & \\
\hline
\end{tabular}

Table S4. Cartesian Coordinates, Relative Energies, and Equilibrium Populations of Low-energy Conformers (>1\%) of $9 S *-3$.

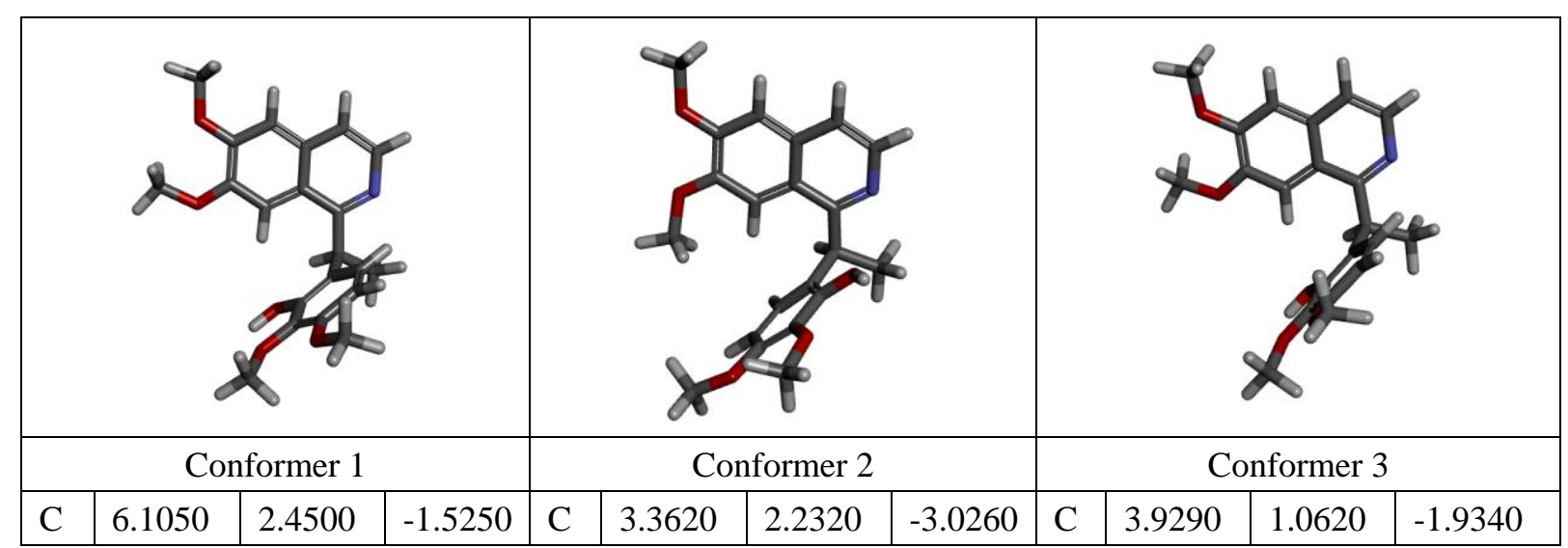




\begin{tabular}{|c|c|c|c|c|c|c|c|c|c|c|c|}
\hline $\mathrm{C}$ & 5.3460 & 3.6480 & -1.3840 & $\mathrm{C}$ & 2.4560 & 2.8880 & -2.1560 & $\mathrm{C}$ & 4.5670 & 1.9220 & -0.9940 \\
\hline $\mathrm{C}$ & 3.9820 & 3.5820 & -1.1800 & $\mathrm{C}$ & 2.5960 & 2.7790 & -0.7890 & $\mathrm{C}$ & 4.1870 & 3.2400 & -0.8910 \\
\hline $\mathrm{H}$ & 3.4240 & 4.4720 & -1.0920 & $\mathrm{H}$ & 1.9080 & 3.2660 & -0.1540 & $\mathrm{H}$ & 4.6740 & 3.8510 & -0.1780 \\
\hline $\mathrm{C}$ & 3.3160 & 2.3280 & -1.0800 & $\mathrm{C}$ & 3.6620 & 2.0210 & -0.2260 & $\mathrm{C}$ & 3.1570 & 3.7750 & -1.7160 \\
\hline $\mathrm{C}$ & 4.0870 & 1.1490 & -1.2060 & $\mathrm{C}$ & 4.5700 & 1.3840 & -1.1070 & $\mathrm{C}$ & 2.5220 & 2.9070 & -2.6360 \\
\hline $\mathrm{C}$ & 5.4810 & 1.2290 & -1.4360 & $\mathrm{C}$ & 4.4050 & 1.4920 & -2.5090 & $\mathrm{C}$ & 2.9210 & 1.5530 & -2.7380 \\
\hline $\mathrm{H}$ & 6.0490 & 0.3420 & -1.5380 & $\mathrm{H}$ & 5.0890 & 1.0010 & -3.1480 & $\mathrm{H}$ & 2.4330 & 0.9300 & -3.4380 \\
\hline $\mathrm{C}$ & 1.9050 & 2.2010 & -0.8480 & $\mathrm{C}$ & 3.8650 & 1.8770 & 1.1870 & $\mathrm{C}$ & 2.7290 & 5.1430 & -1.6610 \\
\hline $\mathrm{N}$ & 1.3390 & 1.0190 & -0.7440 & $\mathrm{~N}$ & 4.8780 & 1.1850 & 1.6590 & $\mathrm{~N}$ & 1.7560 & 5.5810 & -2.4310 \\
\hline $\mathrm{C}$ & 2.0690 & -0.1230 & -0.8620 & $\mathrm{C}$ & 5.7610 & 0.5730 & 0.8250 & $\mathrm{C}$ & 1.1320 & 4.7560 & -3.3120 \\
\hline $\mathrm{H}$ & 1.5530 & -1.0450 & -0.7710 & $\mathrm{H}$ & 6.5560 & 0.0290 & 1.2690 & $\mathrm{H}$ & 0.3560 & 5.1710 & -3.9050 \\
\hline $\mathrm{C}$ & 3.4170 & -0.0900 & -1.0910 & $\mathrm{C}$ & 5.6340 & 0.6520 & -0.5350 & $\mathrm{C}$ & 1.4870 & 3.4410 & -3.4370 \\
\hline $\mathrm{H}$ & 3.9660 & -0.9900 & -1.1810 & $\mathrm{H}$ & 6.3320 & 0.1650 & -1.1640 & $\mathrm{H}$ & 0.9890 & 2.8180 & -4.1320 \\
\hline $\mathrm{C}$ & 1.0280 & 3.4610 & -0.7070 & $\mathrm{C}$ & 2.8930 & 2.5440 & 2.1800 & $\mathrm{C}$ & 3.4000 & 6.1260 & -0.6830 \\
\hline $\mathrm{H}$ & 1.2210 & 4.1050 & -1.5530 & $\mathrm{H}$ & 1.8840 & 2.2830 & 1.8960 & $\mathrm{H}$ & 4.4720 & 6.0170 & -0.7710 \\
\hline $\mathrm{C}$ & -0.4840 & 3.1360 & -0.6820 & $\mathrm{C}$ & 3.1180 & 2.0800 & 3.6380 & $\mathrm{C}$ & 3.0410 & 7.6000 & -0.9840 \\
\hline $\mathrm{H}$ & -1.0300 & 4.0760 & -0.5970 & $\mathrm{H}$ & 4.1280 & 2.3450 & 3.9520 & $\mathrm{H}$ & 1.9650 & 7.7410 & -0.8770 \\
\hline $\mathrm{H}$ & -0.7030 & 2.4930 & 0.1700 & $\mathrm{H}$ & 2.9780 & 1.0000 & 3.6790 & $\mathrm{H}$ & 3.3560 & 7.8280 & -2.0020 \\
\hline $\mathrm{H}$ & -0.7410 & 2.6330 & -1.6140 & $\mathrm{H}$ & 2.3840 & 2.5810 & 4.2690 & $\mathrm{H}$ & 3.5760 & 8.2290 & -0.2720 \\
\hline $\mathrm{C}$ & 1.3590 & 4.1530 & 0.6180 & $\mathrm{C}$ & 3.1130 & 4.0590 & 2.1530 & $\mathrm{C}$ & 2.9170 & 5.8310 & 0.7380 \\
\hline $\mathrm{C}$ & 1.5760 & 5.5340 & 0.6820 & $\mathrm{C}$ & 2.0360 & 4.9480 & 2.0720 & $\mathrm{C}$ & 3.8120 & 5.7920 & 1.8120 \\
\hline $\mathrm{C}$ & 1.8770 & 6.1770 & 1.8830 & $\mathrm{C}$ & 2.2230 & 6.3310 & 2.0510 & $\mathrm{C}$ & 3.3890 & 5.5290 & 3.1160 \\
\hline $\mathrm{C}$ & 1.9660 & 5.4180 & 620 & $\mathrm{C}$ & 3.5280 & 6.8460 & 2.1130 & $\mathrm{C}$ & 2.0240 & 5.3010 & 3.3580 \\
\hline $\mathrm{C}$ & & & & $\mathrm{C}$ & & & & $\mathrm{C}$ & & & \\
\hline $\mathrm{H}$ & 1.8070 & 3.4500 & 3.8910 & $\mathrm{H}$ & 5.6040 & 6.3380 & 2.2470 & $\mathrm{H}$ & 0.0850 & 5.1760 & 2.4590 \\
\hline $\mathrm{C}$ & 1.4450 & 3.4160 & 1.8030 & $\mathrm{C}$ & 4.4040 & 4.5910 & 2.2190 & $\mathrm{C}$ & 1.5620 & 5.6090 & 1.0010 \\
\hline $\mathrm{H}$ & 1.2800 & 2.3680 & 1.7840 & $\mathrm{H}$ & 5.2390 & 3.9390 & 2.2860 & $\mathrm{H}$ & 0.8590 & 5.6410 & 0.2070 \\
\hline $\mathrm{O}$ & 1.5040 & 6.3140 & -0.4380 & $\mathrm{O}$ & 0.7490 & 4.4950 & 2.0060 & $\mathrm{O}$ & 5.1480 & 6.0100 & 1.6220 \\
\hline $\mathrm{H}$ & 1.6300 & 7.2280 & -0.1000 & $\mathrm{H}$ & 0.2080 & 5.3160 & 2.0100 & $\mathrm{H}$ & 5.5360 & 9110 & 2.5190 \\
\hline $\mathrm{O}$ & 7.4730 & & & $\mathrm{O}$ & & & 910 & $\mathrm{O}$ & 170 & -0.2650 & -2.0440 \\
\hline $\mathrm{C}$ & 8.3890 & 3.5140 & -1.8920 & $\mathrm{C}$ & 3.9950 & 1.7610 & -5.4160 & $\mathrm{C}$ & 3.7640 & -1.2480 & -2.9500 \\
\hline $\mathrm{H}$ & 8.3720 & 4.0910 & -0.9670 & $\mathrm{H}$ & 3.9620 & 0.6780 & -5.2950 & $\mathrm{H}$ & 4.2920 & -2.1870 & -2.7840 \\
\hline $\mathrm{H}$ & 8.0650 & 4.1190 & -2.7390 & $\mathrm{H}$ & 5.0110 & 2.1440 & -5.3070 & $\mathrm{H}$ & 3.9200 & -0.9000 & -3.9710 \\
\hline $\mathrm{H}$ & 9.3830 & 3.1050 & -2.0700 & $\mathrm{H}$ & 3.5810 & 2.0500 & -6.3820 & $\mathrm{H}$ & 2.7030 & -1.3620 & -2.7270 \\
\hline $\mathrm{O}$ & 5.9700 & 4.8870 & -1.4440 & $\mathrm{O}$ & & 3.6430 & -2.7130 & $\mathrm{O}$ & 5.5840 & 1.5260 & -0.1360 \\
\hline $\mathrm{C}$ & 5.3410 & 6.1620 & -1.1590 & $\mathrm{C}$ & 0.5170 & 4.4760 & -1.9660 & $\mathrm{C}$ & 6.1640 & 0.2150 & 0.0290 \\
\hline $\mathrm{H}$ & 6.1090 & 6.9330 & -1.2140 & $\mathrm{H}$ & 1.0990 & 5.1950 & -1.3880 & $\mathrm{H}$ & 6.6210 & -0.0770 & -0.9160 \\
\hline $\mathrm{H}$ & 4.9140 & 6.1190 & -0.1560 & $\mathrm{H}$ & -0.0760 & 3.8350 & -1.3140 & $\mathrm{H}$ & 5.3700 & -0.4760 & 0.3120 \\
\hline $\mathrm{H}$ & 4.5680 & 6.3400 & -1.9070 & $\mathrm{H}$ & -0.1230 & 4.9900 & -2.6830 & $\mathrm{H}$ & 6.9150 & 0.2780 & 0.8150 \\
\hline $\mathrm{O}$ & 2.0730 & 7.5700 & 1.7990 & $\mathrm{O}$ & 1.0490 & 7.1060 & 1.9660 & $\mathrm{O}$ & 4.3990 & 5.5170 & 4.0980 \\
\hline $\mathrm{C}$ & 2.3360 & 8.4830 & 2.8900 & $\mathrm{C}$ & 0.9520 & 8.5490 & 1.9570 & $\mathrm{C}$ & 4.2480 & 5.2230 & 5.5050 \\
\hline $\mathrm{H}$ & 1.5140 & 8.4090 & 3.6030 & $\mathrm{H}$ & -0.1020 & 8.8170 & 1.9010 & $\mathrm{H}$ & 3.8300 & 4.2200 & 5.6020 \\
\hline $\mathrm{H}$ & 2.3970 & 9.4910 & 2.4800 & $\mathrm{H}$ & 1.4890 & 8.9220 & 1.0840 & $\mathrm{H}$ & 3.5810 & 5.9670 & 5.9410 \\
\hline $\mathrm{H}$ & 3.2820 & 8.2000 & 3.3530 & $\mathrm{H}$ & 1.3950 & 8.9270 & 2.8790 & $\mathrm{H}$ & 5.2330 & 5.2740 & 5.9690 \\
\hline $\mathrm{O}$ & 2.2720 & 6.0260 & 4.2740 & $\mathrm{O}$ & 3.7510 & 8.2190 & 2.0910 & $\mathrm{O}$ & 1.5670 & 5.0360 & 4.6450 \\
\hline
\end{tabular}




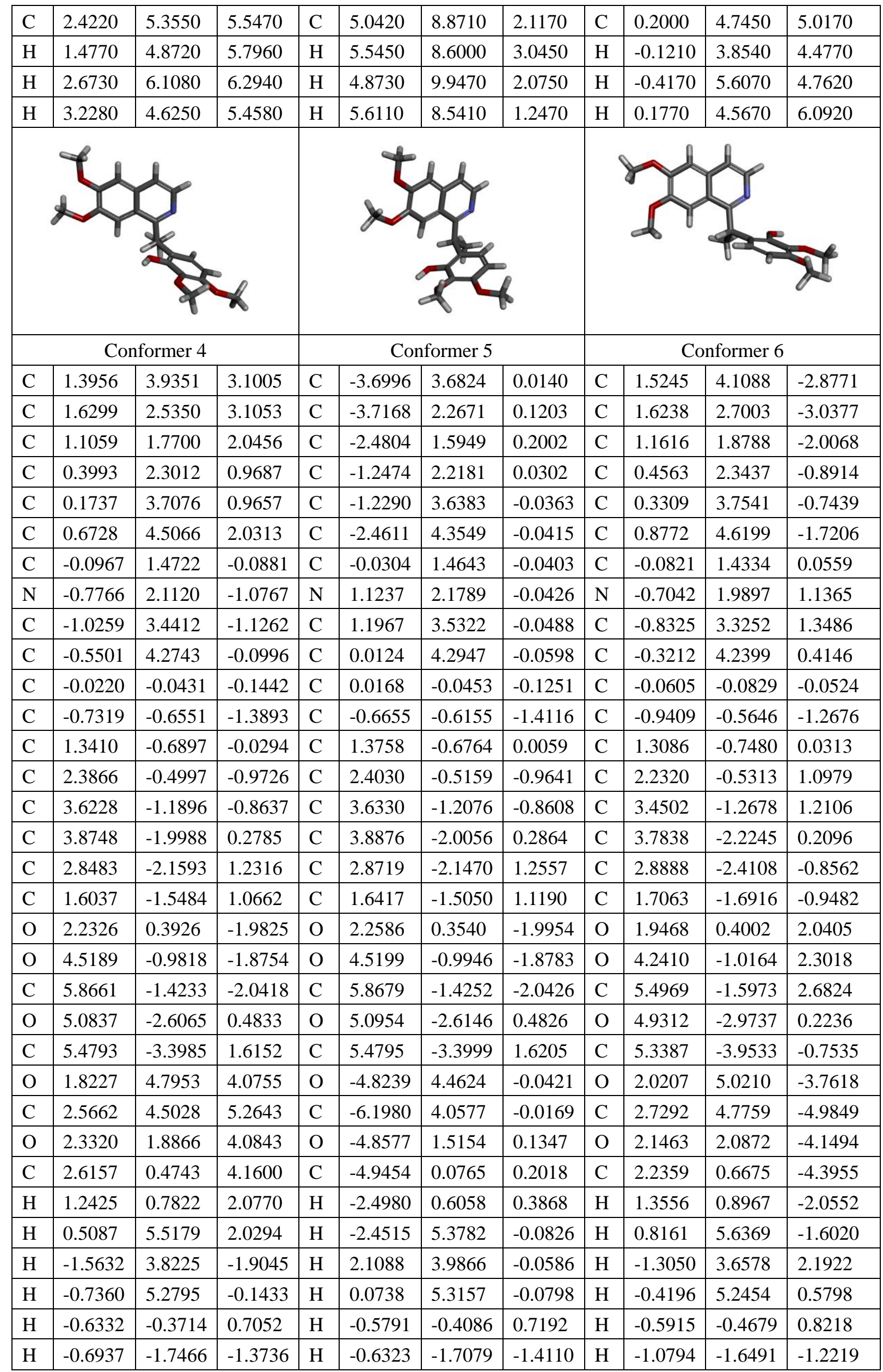




\begin{tabular}{|l|l|l|l|l|l|l|l|l|l|l|l|}
\hline $\mathrm{H}$ & -0.2605 & -0.3065 & -2.3092 & $\mathrm{H}$ & -0.1700 & -0.2551 & -2.3100 & $\mathrm{H}$ & -0.4823 & -0.3466 & -2.2348 \\
\hline $\mathrm{H}$ & -1.7809 & -0.3545 & -1.4099 & $\mathrm{H}$ & -1.7115 & -0.3328 & -1.4982 & $\mathrm{H}$ & -1.9275 & -0.1087 & -1.2690 \\
\hline $\mathrm{H}$ & 2.9941 & -2.7226 & 2.0700 & $\mathrm{H}$ & 3.0121 & -2.7207 & 2.0864 & $\mathrm{H}$ & 3.0790 & -3.0827 & -1.5980 \\
\hline $\mathrm{H}$ & 0.9014 & -1.7109 & 1.7929 & $\mathrm{H}$ & 0.9469 & -1.6542 & 1.8557 & $\mathrm{H}$ & 1.1482 & -1.9017 & -1.7710 \\
\hline $\mathrm{H}$ & 1.4171 & 0.9253 & -2.0628 & $\mathrm{H}$ & 1.4685 & 0.9252 & -2.0596 & $\mathrm{H}$ & 2.5804 & 0.5639 & 2.7728 \\
\hline $\mathrm{H}$ & 6.2571 & -1.0360 & -2.9829 & $\mathrm{H}$ & 6.2594 & -1.0357 & -2.9825 & $\mathrm{H}$ & 5.8452 & -1.1522 & 3.6146 \\
\hline $\mathrm{H}$ & 5.9159 & -2.5127 & -2.0571 & $\mathrm{H}$ & 5.9190 & -2.5138 & -2.0562 & $\mathrm{H}$ & 6.2444 & -1.4129 & 1.9090 \\
\hline $\mathrm{H}$ & 6.4722 & -1.0339 & -1.2230 & $\mathrm{H}$ & 6.4675 & -1.0314 & -1.2211 & $\mathrm{H}$ & 5.3841 & -2.6728 & 2.8311 \\
\hline $\mathrm{H}$ & 6.5070 & -3.7413 & 1.5118 & $\mathrm{H}$ & 6.5051 & -3.7494 & 1.5292 & $\mathrm{H}$ & 6.2996 & -4.3942 & -0.5003 \\
\hline $\mathrm{H}$ & 4.8245 & -4.2682 & 1.7019 & $\mathrm{H}$ & 4.8162 & -4.2636 & 1.7026 & $\mathrm{H}$ & 5.4213 & -3.4772 & -1.7343 \\
\hline $\mathrm{H}$ & 5.4015 & -2.7945 & 2.5226 & $\mathrm{H}$ & 5.3948 & -2.7886 & 2.5226 & $\mathrm{H}$ & 4.5914 & -4.7496 & -0.8050 \\
\hline $\mathrm{H}$ & 2.7413 & 5.4195 & 5.8271 & $\mathrm{H}$ & -6.8458 & 4.9330 & -0.0619 & $\mathrm{H}$ & 3.0238 & 5.7201 & -5.4409 \\
\hline $\mathrm{H}$ & 3.5305 & 4.0631 & 5.0031 & $\mathrm{H}$ & -6.4122 & 3.5124 & 0.9043 & $\mathrm{H}$ & 2.0887 & 4.2338 & -5.6825 \\
\hline $\mathrm{H}$ & 2.0062 & 3.8077 & 5.8924 & $\mathrm{H}$ & -6.4117 & 3.4200 & -0.8765 & $\mathrm{H}$ & 3.6284 & 4.1895 & -4.7840 \\
\hline $\mathrm{H}$ & 3.1880 & 0.2297 & 5.0496 & $\mathrm{H}$ & -5.9757 & -0.2646 & 0.2054 & $\mathrm{H}$ & 2.7097 & 0.4592 & -5.3498 \\
\hline $\mathrm{H}$ & 3.1893 & 0.1727 & 3.2792 & $\mathrm{H}$ & -4.4614 & -0.2769 & 1.1165 & $\mathrm{H}$ & 1.2298 & 0.2373 & -4.3982 \\
\hline $\mathrm{H}$ & 1.6764 & -0.0855 & 4.1851 & $\mathrm{H}$ & -4.4329 & -0.3541 & -0.6637 & $\mathrm{H}$ & 2.8255 & 0.1876 & -3.6092 \\
\hline
\end{tabular}

Table S5. Cartesian Coordinates, Relative Energies, and Equilibrium Populations of Low-energy

Conformers (>1\%) of 9S*-4.

\begin{tabular}{|c|c|c|c|c|c|c|c|c|c|c|c|}
\hline \multicolumn{4}{|c|}{ Conformer 1} & \multicolumn{4}{|c|}{ Conformer 2} & \multicolumn{4}{|c|}{ Conformer 3} \\
\hline $\mathrm{C}$ & 3.4362 & -1.1103 & -0.8153 & $\mathrm{C}$ & -3.3915 & -1.1548 & 0.3260 & $\mathrm{C}$ & 3.4096 & -1.2296 & -0.2447 \\
\hline $\mathrm{C}$ & 2.4769 & -1.4272 & 0.1737 & $\mathrm{C}$ & -2.4128 & -1.3005 & -0.6830 & $\mathrm{C}$ & 2.3954 & -1.3543 & 0.7319 \\
\hline $\mathrm{C}$ & 1.6649 & -0.4149 & 0.6884 & $\mathrm{C}$ & -1.5750 & -0.2346 & -0.9890 & $\mathrm{C}$ & 1.5724 & -0.2704 & 1.0119 \\
\hline $\mathrm{C}$ & 1.7775 & 0.9104 & 0.2282 & $\mathrm{C}$ & -1.6754 & 0.9954 & -0.3140 & $\mathrm{C}$ & 1.7220 & 0.9573 & 0.3417 \\
\hline $\mathrm{C}$ & 2.7200 & 1.2168 & -0.7698 & $\mathrm{C}$ & -2.6421 & 1.1299 & 0.6994 & $\mathrm{C}$ & 2.7260 & 1.0724 & -0.6372 \\
\hline $\mathrm{C}$ & 3.5338 & 0.2014 & -1.2762 & $\mathrm{C}$ & -3.4856 & 0.0606 & 1.0138 & $\mathrm{C}$ & 3.5557 & -0.0150 & -0.9250 \\
\hline $\mathrm{C}$ & 0.9324 & 2.0227 & 0.7566 & $\mathrm{C}$ & -0.8113 & 2.1723 & -0.6217 & $\mathrm{C}$ & 0.8675 & 2.1488 & 0.6153 \\
\hline $\mathrm{N}$ & 1.2311 & 3.2621 & 0.5980 & $\mathrm{~N}$ & -1.1044 & 3.3690 & -0.2559 & $\mathrm{~N}$ & 1.1927 & 3.3400 & 0.2590 \\
\hline $\mathrm{C}$ & 2.4669 & 3.5945 & -0.1136 & $\mathrm{C}$ & -2.3544 & 3.5835 & 0.4747 & $\mathrm{C}$ & 2.4740 & 3.5316 & -0.4225 \\
\hline $\mathrm{C}$ & 2.7837 & 2.6404 & -1.2658 & $\mathrm{C}$ & -2.7036 & 2.4459 & 1.4356 & $\mathrm{C}$ & 2.8398 & 2.3873 & -1.3694 \\
\hline $\mathrm{C}$ & -0.3440 & 1.6817 & 1.5290 & $\mathrm{C}$ & 0.4802 & 1.9524 & -1.4108 & $\mathrm{C}$ & -0.4565 & 1.9498 & 1.3556 \\
\hline $\mathrm{C}$ & -0.9341 & 2.8930 & 2.2728 & $\mathrm{C}$ & 1.0804 & 3.2611 & -1.9537 & $\mathrm{C}$ & -1.0714 & 3.2714 & 1.8480 \\
\hline $\mathrm{C}$ & -1.3844 & 1.0374 & 0.6158 & $\mathrm{C}$ & 1.5023 & 1.1802 & -0.5793 & $\mathrm{C}$ & -1.4460 & 1.1634 & 0.4984 \\
\hline $\mathrm{C}$ & -1.7678 & 1.6226 & -0.5933 & $\mathrm{C}$ & 1.8844 & 1.6020 & 0.6962 & $\mathrm{C}$ & -1.7809 & 1.5665 & -0.7964 \\
\hline $\mathrm{C}$ & -2.7441 & 1.0632 & -1.4233 & $\mathrm{C}$ & 2.8605 & 0.9384 & 1.4464 & $\mathrm{C}$ & -2.7036 & 0.8722 & -1.5853 \\
\hline $\mathrm{C}$ & -3.3789 & -0.1267 & -1.0479 & $\mathrm{C}$ & 3.4907 & -0.1953 & 0.9197 & $\mathrm{C}$ & -3.3290 & -0.2731 & -1.0782 \\
\hline
\end{tabular}




\begin{tabular}{|c|c|c|c|c|c|c|c|c|c|c|c|}
\hline $\mathrm{C}$ & -3.0329 & -0.7208 & 0.1772 & $\mathrm{C}$ & 3.1061 & -0.6567 & -0.3502 & $\mathrm{C}$ & -3.0297 & -0.6857 & 0.2308 \\
\hline $\mathrm{C}$ & -2.0387 & -0.1486 & 0.9825 & $\mathrm{C}$ & 2.1371 & 0.0365 & -1.0890 & $\mathrm{C}$ & -2.0917 & 0.0207 & 0.9965 \\
\hline $\mathrm{O}$ & -1.7006 & -0.7851 & 2.1482 & $\mathrm{O}$ & 1.8151 & -0.4205 & -2.3373 & $\mathrm{O}$ & -1.8000 & -0.4323 & 2.2539 \\
\hline $\mathrm{O}$ & 4.2259 & -2.0664 & -1.4029 & $\mathrm{O}$ & -4.1967 & -2.2334 & 0.5496 & $\mathrm{O}$ & 4.1934 & -2.3276 & -0.4465 \\
\hline $\mathrm{C}$ & 5.1738 & -2.7323 & -0.5571 & $\mathrm{C}$ & -5.2310 & -2.1224 & 1.5208 & $\mathrm{C}$ & 5.2572 & -2.2420 & -1.3876 \\
\hline $\mathrm{O}$ & 2.4054 & -2.7409 & 0.5506 & $\mathrm{O}$ & -2.3242 & -2.4524 & -1.4317 & $\mathrm{O}$ & 2.2557 & -2.5072 & 1.4703 \\
\hline $\mathrm{C}$ & 1.4122 & -3.1211 & 1.4973 & $\mathrm{C}$ & -1.8226 & -3.6064 & -0.7488 & $\mathrm{C}$ & 1.7166 & -3.6343 & 0.7685 \\
\hline $\mathrm{O}$ & -4.3263 & -0.7780 & -1.7901 & $\mathrm{O}$ & 4.4746 & -0.9090 & 1.5501 & $\mathrm{O}$ & -4.2264 & -1.0458 & -1.7651 \\
\hline $\mathrm{C}$ & -4.6696 & -0.2406 & -3.0630 & $\mathrm{C}$ & 4.9379 & -0.4438 & 2.8127 & $\mathrm{C}$ & -4.5329 & -0.6864 & -3.1075 \\
\hline $\mathrm{O}$ & -3.5592 & -1.9198 & 0.6265 & $\mathrm{O}$ & 3.6981 & -1.7343 & -0.9856 & $\mathrm{O}$ & -3.5499 & -1.8300 & 0.8101 \\
\hline $\mathrm{C}$ & -4.9696 & -1.9275 & 0.9140 & $\mathrm{C}$ & 3.5067 & -3.0194 & -0.3688 & $\mathrm{C}$ & -4.9677 & -1.8262 & 1.0565 \\
\hline $\mathrm{H}$ & 0.9264 & -0.6603 & 1.4400 & $\mathrm{H}$ & -0.8469 & -0.3933 & -1.7767 & $\mathrm{H}$ & 0.8140 & -0.4139 & 1.7733 \\
\hline $\mathrm{H}$ & 4.2677 & 0.4116 & -2.0494 & $\mathrm{H}$ & -4.2279 & 0.1924 & 1.7934 & $\mathrm{H}$ & 4.3266 & 0.1009 & -1.6791 \\
\hline $\mathrm{H}$ & 3.2894 & 3.5831 & 0.6195 & $\mathrm{H}$ & -3.1604 & 3.7091 & -0.2660 & $\mathrm{H}$ & 3.2529 & 3.6425 & 0.3489 \\
\hline $\mathrm{H}$ & 2.3814 & 4.6247 & -0.4737 & $\mathrm{H}$ & -2.2723 & 4.5330 & 1.0133 & $\mathrm{H}$ & 2.4303 & 4.4822 & -0.9637 \\
\hline $\mathrm{H}$ & 3.7671 & 2.8647 & -1.6936 & $\mathrm{H}$ & -3.6927 & 2.6045 & 1.8802 & $\mathrm{H}$ & 3.8483 & 2.5281 & -1.7746 \\
\hline $\mathrm{H}$ & 2.0459 & 2.7858 & -2.0702 & $\mathrm{H}$ & -1.9785 & 2.4377 & 2.2642 & $\mathrm{H}$ & 2.1480 & 2.3921 & -2.2261 \\
\hline $\mathrm{H}$ & -0.0766 & 0.9390 & 2.2876 & $\mathrm{H}$ & 0.2316 & 1.3288 & -2.2749 & $\mathrm{H}$ & -0.2445 & 1.3421 & 2.2407 \\
\hline $\mathrm{H}$ & -1.2286 & 3.6800 & 1.5748 & $\mathrm{H}$ & 1.3557 & 3.9375 & -1.1413 & $\mathrm{H}$ & -1.3121 & 3.9325 & 1.0122 \\
\hline $\mathrm{H}$ & -0.1996 & 3.3207 & 2.9610 & $\mathrm{H}$ & 0.3589 & 3.7840 & -2.5882 & $\mathrm{H}$ & -0.3727 & 3.8047 & 2.4989 \\
\hline $\mathrm{H}$ & -1.8143 & 2.5784 & 2.8427 & $\mathrm{H}$ & 1.9741 & 3.0355 & -2.5441 & $\mathrm{H}$ & -1.9885 & 3.0616 & 2.4078 \\
\hline $\mathrm{H}$ & -1.2836 & 2.5445 & -0.9024 & $\mathrm{H}$ & 1.4094 & 2.4835 & 1.1173 & $\mathrm{H}$ & -1.3003 & 2.4497 & -1.2074 \\
\hline $\mathrm{H}$ & -2.9926 & 1.5519 & -2.3572 & $\mathrm{H}$ & 3.1272 & 1.3153 & 2.4260 & $\mathrm{H}$ & -2.9181 & 1.2225 & -2.5874 \\
\hline $\mathrm{H}$ & -2.2468 & -1.5891 & 2.1860 & $\mathrm{H}$ & 2.4059 & -1.1706 & -2.5200 & $\mathrm{H}$ & -2.2940 & -1.2615 & 2.3719 \\
\hline $\mathrm{H}$ & 5.8609 & -2.0045 & -0.1068 & $\mathrm{H}$ & -4.8224 & -1.9378 & 2.5221 & $\mathrm{H}$ & 4.8819 & -2.0446 & -2.3996 \\
\hline $\mathrm{H}$ & 5.7342 & -3.4038 & -1.2103 & $\mathrm{H}$ & -5.7492 & -3.0820 & 1.5114 & $\mathrm{H}$ & 5.7494 & -3.2150 & -1.3665 \\
\hline $\mathrm{H}$ & 4.6737 & -3.3074 & 0.2264 & $\mathrm{H}$ & -5.9373 & -1.3247 & 1.2603 & $\mathrm{H}$ & 5.9768 & -1.4638 & -1.1053 \\
\hline $\mathrm{H}$ & 1.5702 & -2.6266 & 2.4639 & $\mathrm{H}$ & -0.8098 & -3.4184 & -0.3688 & $\mathrm{H}$ & 0.7145 & -3.4052 & 0.3844 \\
\hline $\mathrm{H}$ & 1.5191 & -4.1999 & 1.6189 & $\mathrm{H}$ & -1.7891 & -4.4056 & -1.4919 & $\mathrm{H}$ & 1.6495 & -4.4412 & 1.5009 \\
\hline $\mathrm{H}$ & 0.4048 & -2.8906 & 1.1322 & $\mathrm{H}$ & -2.4798 & -3.8988 & 0.0759 & $\mathrm{H}$ & 2.3705 & -3.9393 & -0.0546 \\
\hline $\mathrm{H}$ & -5.1079 & 0.7606 & -2.9696 & $\mathrm{H}$ & 5.3482 & 0.5704 & 2.7364 & $\mathrm{H}$ & -4.9967 & 0.3063 & -3.1603 \\
\hline $\mathrm{H}$ & -5.4097 & -0.9233 & -3.4825 & $\mathrm{H}$ & 5.7287 & -1.1336 & 3.1105 & $\mathrm{H}$ & -5.2408 & -1.4373 & -3.4610 \\
\hline $\mathrm{H}$ & -3.7961 & -0.1983 & -3.7246 & $\mathrm{H}$ & 4.1380 & -0.4595 & 3.5635 & $\mathrm{H}$ & -3.6362 & -0.7053 & -3.7389 \\
\hline $\mathrm{H}$ & -5.2171 & -1.1446 & 1.6411 & $\mathrm{H}$ & 3.9995 & -3.0573 & 0.6053 & $\mathrm{H}$ & -5.2466 & -0.9682 & 1.6802 \\
\hline $\mathrm{H}$ & -5.1828 & -2.9093 & 1.3412 & $\mathrm{H}$ & 3.9598 & -3.7471 & -1.0449 & $\mathrm{H}$ & -5.1821 & -2.7548 & 1.5892 \\
\hline $\mathrm{H}$ & -5.5495 & -1.7839 & -0.0003 & $\mathrm{H}$ & 2.4372 & -3.2353 & -0.2553 & $\mathrm{H}$ & -5.5220 & -1.7992 & 0.1156 \\
\hline \multicolumn{4}{|c|}{ Conformer 4} & \multicolumn{4}{|c|}{ Conformer 5} & \multicolumn{4}{|c|}{ Conformer 6} \\
\hline $\mathrm{C}$ & 3.4323 & 1.0907 & 0.6942 & $\mathrm{C}$ & -3.3594 & -1.1156 & 0.5544 & $\mathrm{C}$ & 4.6436 & 0.3128 & -0.7394 \\
\hline $\mathrm{C}$ & 2.5593 & 1.3017 & -0.3973 & $\mathrm{C}$ & -2.4042 & -1.3609 & -0.4580 & $\mathrm{C}$ & 4.0556 & -0.7728 & -0.0511 \\
\hline $\mathrm{C}$ & 1.7265 & 0.2634 & -0.8178 & $\mathrm{C}$ & -1.5583 & -0.3378 & -0.8691 & $\mathrm{C}$ & 2.7136 & -0.6959 & 0.3254 \\
\hline
\end{tabular}




\begin{tabular}{|c|c|c|c|c|c|c|c|c|c|c|c|}
\hline $\mathrm{C}$ & 1.7661 & -0.9971 & -0.1935 & $\mathrm{C}$ & -1.6400 & 0.9509 & -0.3107 & $\mathrm{C}$ & 1.9329 & 0.4370 & 0.0248 \\
\hline $\mathrm{C}$ & 2.6547 & -1.2099 & 0.8759 & $\mathrm{C}$ & -2.6017 & 1.1928 & 0.6871 & $\mathrm{C}$ & 2.5202 & 1.5066 & -0.6759 \\
\hline $\mathrm{C}$ & 3.4674 & -0.1601 & 1.3083 & $\mathrm{C}$ & -3.4481 & 0.1651 & 1.1124 & $\mathrm{C}$ & 3.8640 & 1.4259 & -1.0480 \\
\hline $\mathrm{C}$ & 0.9091 & -2.1401 & -0.6287 & $\mathrm{C}$ & -0.7606 & 2.0813 & -0.7281 & $\mathrm{C}$ & 0.4885 & 0.5537 & 0.3932 \\
\hline $\mathrm{N}$ & 1.1661 & -3.3605 & -0.3209 & $\mathrm{~N}$ & -1.0369 & 3.3114 & -0.4785 & $\mathrm{~N}$ & -0.1428 & 1.6699 & 0.3914 \\
\hline $\mathrm{C}$ & 2.3627 & -3.6392 & 0.4760 & $\mathrm{C}$ & -2.2836 & 3.6105 & 0.2279 & $\mathrm{C}$ & 0.5948 & 2.8885 & 0.0599 \\
\hline $\mathrm{C}$ & 2.6564 & -2.5702 & 1.5294 & $\mathrm{C}$ & -2.6469 & 2.5746 & 1.2929 & $\mathrm{C}$ & 1.6460 & 2.6841 & -1.0310 \\
\hline $\mathrm{C}$ & -0.3328 & -1.8550 & -1.4763 & $\mathrm{C}$ & 0.5272 & 1.7683 & -1.4924 & $\mathrm{C}$ & -0.2814 & -0.7098 & 0.8129 \\
\hline $\mathrm{C}$ & -0.9010 & -3.1176 & -2.1479 & $\mathrm{C}$ & 1.1577 & 3.0143 & -2.1380 & $\mathrm{C}$ & -0.1203 & -0.9690 & 2.3309 \\
\hline $\mathrm{C}$ & -1.4007 & -1.1418 & -0.6489 & $\mathrm{C}$ & 1.5268 & 1.0404 & -0.5962 & $\mathrm{C}$ & -1.7254 & -0.7009 & 0.3241 \\
\hline $\mathrm{C}$ & -1.8794 & -1.6797 & 0.5484 & $\mathrm{C}$ & 1.9351 & 1.5687 & 0.6307 & $\mathrm{C}$ & -2.0316 & -1.3560 & -0.8727 \\
\hline $\mathrm{C}$ & -2.9001 & -1.0763 & 1.2891 & $\mathrm{C}$ & 2.8671 & 0.9272 & 1.4525 & $\mathrm{C}$ & -3.3153 & -1.3913 & -1.4263 \\
\hline $\mathrm{C}$ & -3.4793 & 0.1144 & 0.8345 & $\mathrm{C}$ & 3.4264 & -0.2915 & 1.0495 & $\mathrm{C}$ & -4.3633 & -0.7445 & -0.7646 \\
\hline $\mathrm{C}$ & -2.9962 & 0.6925 & -0.3509 & $\mathrm{C}$ & 3.0525 & -0.8321 & -0.1918 & $\mathrm{C}$ & -4.0872 & -0.0655 & 0.4320 \\
\hline $\mathrm{C}$ & -1.9819 & 0.0595 & -1.0827 & $\mathrm{C}$ & 2.1058 & -0.1760 & -0.9908 & $\mathrm{C}$ & -2.7934 & -0.0565 & 0.9780 \\
\hline $\mathrm{O}$ & -1.5648 & 0.6416 & -2.2516 & $\mathrm{O}$ & 1.7399 & -0.7520 & -2.1764 & $\mathrm{O}$ & -2.6184 & 0.5797 & 2.1696 \\
\hline $\mathrm{O}$ & 4.3173 & 2.0499 & 1.1194 & $\mathrm{O}$ & -4.1383 & -2.1738 & 0.9210 & $\mathrm{O}$ & 5.9385 & 0.2767 & -1.1911 \\
\hline $\mathrm{C}$ & 3.7551 & 3.2432 & 1.6818 & $\mathrm{C}$ & -5.0754 & -1.9970 & 1.9770 & $\mathrm{C}$ & 6.9696 & 0.2408 & -0.1943 \\
\hline $\mathrm{O}$ & 2.6055 & 2.5384 & -0.9833 & $\mathrm{O}$ & -2.2376 & -2.6193 & -0.9901 & $\mathrm{O}$ & 4.8653 & -1.8511 & 0.1870 \\
\hline $\mathrm{C}$ & 1.8347 & 2.7582 & -2.1600 & $\mathrm{C}$ & -3.2799 & -3.0697 & -1.8630 & $\mathrm{C}$ & 4.3076 & -2.9920 & 0.8273 \\
\hline $\mathrm{O}$ & -4.5003 & 0.7781 & 1.4593 & $\mathrm{O}$ & 4.3264 & -1.0202 & 1.7796 & $\mathrm{O}$ & -5.6651 & -0.7206 & -1.1885 \\
\hline $\mathrm{C}$ & -5.0576 & 0.2004 & 2.6350 & $\mathrm{C}$ & 4.6994 & -0.5349 & 3.0640 & $\mathrm{C}$ & -5.9952 & -1.4296 & -2.3766 \\
\hline $\mathrm{O}$ & -3.5275 & 1.8378 & -0.9183 & $\mathrm{O}$ & 3.5034 & -2.0527 & -0.6614 & $\mathrm{O}$ & -5.0670 & 0.5584 & 1.1866 \\
\hline $\mathrm{C}$ & -3.3797 & 3.0516 & -0.1597 & $\mathrm{C}$ & 4.9071 & -2.1347 & -0.9657 & $\mathrm{C}$ & -5.6603 & 1.7288 & 0.5959 \\
\hline $\mathrm{H}$ & 1.0461 & & & $\mathrm{H}$ & & -0.5830 & -1.6286 & $\mathrm{H}$ & 2.2680 & -1.5271 & 0.8544 \\
\hline $\mathrm{H}$ & 4.1624 & -0.2989 & 2.1319 & $\mathrm{H}$ & -4.1750 & 0.3744 & 1.8897 & $\mathrm{H}$ & 4.3365 & 2.2363 & -1.5961 \\
\hline $\mathrm{H}$ & 3.2148 & -3.7276 & -0.2167 & $\mathrm{H}$ & -3.0888 & 3.6749 & -0.5218 & $\mathrm{H}$ & 1.0757 & 3.2524 & 0.9824 \\
\hline $\mathrm{H}$ & 2.2347 & -4.6207 & 0.9435 & $\mathrm{H}$ & -2.1893 & 4.6061 & 0.6732 & $\mathrm{H}$ & -0.1301 & 3.6523 & -0.2388 \\
\hline $\mathrm{H}$ & 3.6142 & -2.7676 & 2.0234 & $\mathrm{H}$ & -3.6340 & 2.7878 & 1.7186 & $\mathrm{H}$ & 2.2451 & 3.5915 & -1.1663 \\
\hline $\mathrm{H}$ & 1.8809 & -2.6088 & 2.3101 & $\mathrm{H}$ & -1.9224 & 2.6367 & 2.1196 & $\mathrm{H}$ & 1.1396 & 2.4902 & -1.9892 \\
\hline $\mathrm{H}$ & -0.0356 & -1.1720 & -2.2776 & $\mathrm{H}$ & 0.2643 & 1.0819 & -2.3031 & $\mathrm{H}$ & 0.1886 & -1.5528 & 0.2932 \\
\hline $\mathrm{H}$ & -1.2231 & -3.8509 & -1.4053 & $\mathrm{H}$ & 1.4498 & 3.7478 & -1.3830 & $\mathrm{H}$ & -0.5137 & -0.1356 & 2.9124 \\
\hline $\mathrm{H}$ & -0.1434 & -3.5957 & -2.7756 & $\mathrm{H}$ & 0.4485 & 3.5018 & -2.8132 & $\mathrm{H}$ & 0.9336 & -1.1060 & 2.5932 \\
\hline $\mathrm{H}$ & -1.7587 & -2.8457 & -2.7715 & $\mathrm{H}$ & 2.0457 & 2.7210 & -2.7072 & $\mathrm{H}$ & -0.6643 & -1.8750 & 2.6185 \\
\hline $\mathrm{H}$ & -1.4455 & -2.6067 & 0.9122 & $\mathrm{H}$ & 1.5062 & 2.5105 & 0.9611 & $\mathrm{H}$ & -1.2303 & -1.8644 & -1.4031 \\
\hline $\mathrm{H}$ & -3.2411 & -1.5434 & 2.2047 & $\mathrm{H}$ & 3.1398 & 1.3753 & 2.4000 & $\mathrm{H}$ & -3.4838 & -1.9260 & -2.3527 \\
\hline $\mathrm{H}$ & -2.1564 & 1.3985 & -2.4037 & $\mathrm{H}$ & 2.1829 & -1.6169 & -2.2125 & $\mathrm{H}$ & -3.4996 & 0.8862 & 2.4447 \\
\hline $\mathrm{H}$ & 3.1623 & 3.7904 & 0.9443 & $\mathrm{H}$ & -4.5750 & -1.7047 & 2.9083 & $\mathrm{H}$ & 6.9019 & 1.1218 & 0.4565 \\
\hline $\mathrm{H}$ & 4.6044 & 3.8508 & 1.9996 & $\mathrm{H}$ & -5.5545 & -2.9674 & 2.1124 & $\mathrm{H}$ & 7.9133 & 0.2661 & -0.7420 \\
\hline $\mathrm{H}$ & 3.1352 & 2.9987 & 2.5540 & $\mathrm{H}$ & -5.8345 & -1.2483 & 1.7182 & $\mathrm{H}$ & 6.9125 & -0.6714 & 0.4052 \\
\hline $\mathrm{H}$ & 2.1269 & 2.0665 & -2.9597 & $\mathrm{H}$ & -4.2373 & -3.1393 & -1.3371 & $\mathrm{H}$ & 3.9541 & -2.7531 & 1.8383 \\
\hline $\mathrm{H}$ & 2.0519 & 3.7820 & -2.4677 & $\mathrm{H}$ & -2.9781 & -4.0610 & -2.2068 & $\mathrm{H}$ & 5.1164 & -3.7211 & 0.8898 \\
\hline $\mathrm{H}$ & 0.7610 & 2.6516 & -1.9652 & $\mathrm{H}$ & -3.3764 & -2.3980 & -2.7260 & $\mathrm{H}$ & 3.4821 & -3.4150 & 0.2414 \\
\hline $\mathrm{H}$ & -5.4707 & -0.7948 & 2.4310 & $\mathrm{H}$ & 5.2003 & 0.4387 & 2.9944 & $\mathrm{H}$ & -5.4606 & -1.0261 & -3.2457 \\
\hline $\mathrm{H}$ & -5.8613 & 0.8709 & 2.9418 & $\mathrm{H}$ & 5.3938 & -1.2723 & 3.4691 & $\mathrm{H}$ & -7.0686 & -1.2931 & -2.5152 \\
\hline
\end{tabular}




\begin{tabular}{|l|l|l|l|l|l|l|l|l|l|l|l|}
\hline $\mathrm{H}$ & -4.3135 & 0.1308 & 3.4380 & $\mathrm{H}$ & 3.8288 & -0.4520 & 3.7259 & $\mathrm{H}$ & -5.7742 & -2.4994 & -2.2762 \\
\hline $\mathrm{H}$ & -3.9578 & 3.0002 & 0.7655 & $\mathrm{H}$ & 5.1882 & -1.3637 & -1.6934 & $\mathrm{H}$ & -4.8884 & 2.4684 & 0.3515 \\
\hline $\mathrm{H}$ & -3.7639 & 3.8512 & -0.7960 & $\mathrm{H}$ & 5.0627 & -3.1248 & -1.3985 & $\mathrm{H}$ & -6.3373 & 2.1358 & 1.3497 \\
\hline $\mathrm{H}$ & -2.3230 & 3.2348 & 0.0701 & $\mathrm{H}$ & 5.5051 & -2.0263 & -0.0579 & $\mathrm{H}$ & -6.2211 & 1.4629 & -0.3033 \\
\hline
\end{tabular}

Table S6. Cartesian Coordinates, Relative Energies, and Equilibrium Populations of Low-energy

Conformers $(>1 \%)$ of $9 R *-5$.

\begin{tabular}{|c|c|c|c|c|c|c|c|}
\hline \multicolumn{4}{|c|}{ Conformer 1} & \multicolumn{4}{|c|}{ Conformer 2} \\
\hline $\mathrm{C}$ & 2.9030 & -1.8690 & 1.7780 & $\mathrm{C}$ & 2.8310 & -1.4980 & 1.7130 \\
\hline $\mathrm{C}$ & 2.5970 & -1.1000 & 0.6120 & $\mathrm{C}$ & 2.6320 & -1.0490 & 0.3710 \\
\hline $\mathrm{C}$ & 1.2740 & -1.1320 & 0.0860 & $\mathrm{C}$ & 1.3580 & -1.1900 & -0.2300 \\
\hline $\mathrm{C}$ & 0.2530 & -1.9050 & 0.7010 & $\mathrm{C}$ & 0.2630 & -1.7550 & 0.4740 \\
\hline $\mathrm{C}$ & 0.5600 & -2.6160 & 1.8920 & $\mathrm{C}$ & 0.4650 & -2.1880 & 1.8110 \\
\hline $\mathrm{C}$ & 1.8760 & -2.6090 & 2.4070 & $\mathrm{C}$ & 1.7360 & -2.0670 & 2.4190 \\
\hline $\mathrm{C}$ & -1.1510 & -1.9810 & 0.1230 & $\mathrm{C}$ & -1.1100 & -1.9090 & -0.1630 \\
\hline $\mathrm{N}$ & -1.9750 & -2.8680 & 0.5860 & $\mathrm{~N}$ & -1.9720 & -2.6950 & 0.4020 \\
\hline $\mathrm{C}$ & -1.6160 & -3.8540 & 1.6350 & $\mathrm{C}$ & -1.6830 & -3.4730 & 1.6330 \\
\hline $\mathrm{C}$ & -0.5220 & -3.3460 & 2.5930 & $\mathrm{C}$ & -0.6820 & -2.7560 & 2.5590 \\
\hline $\mathrm{C}$ & -1.6460 & -1.0470 & -1.0150 & $\mathrm{C}$ & -1.5280 & -1.1620 & -1.4540 \\
\hline $\mathrm{C}$ & -1.5220 & 0.4410 & -0.6750 & $\mathrm{C}$ & -1.2930 & 0.3430 & -1.2890 \\
\hline $\mathrm{C}$ & -1.5270 & 1.4400 & -1.6870 & $\mathrm{C}$ & -1.9730 & 1.0440 & -0.2610 \\
\hline $\mathrm{C}$ & -1.3780 & 2.8110 & -1.3660 & $\mathrm{C}$ & -1.8010 & 2.4380 & -0.0830 \\
\hline $\mathrm{C}$ & -1.2260 & 3.2020 & -0.0080 & $\mathrm{C}$ & -0.9240 & 3.1540 & -0.9420 \\
\hline $\mathrm{C}$ & -1.2620 & 2.2110 & 0.9940 & $\mathrm{C}$ & -0.2380 & 2.4480 & -1.9530 \\
\hline $\mathrm{C}$ & -1.4170 & 0.8610 & 0.6670 & $\mathrm{C}$ & -0.4150 & 1.0720 & -2.1190 \\
\hline $\mathrm{O}$ & -1.6830 & 1.0770 & -2.9500 & $\mathrm{O}$ & -2.7780 & 0.3720 & 0.5460 \\
\hline $\mathrm{O}$ & -1.3960 & 3.6580 & -2.3960 & $\mathrm{O}$ & -2.5000 & 2.9900 & 0.9100 \\
\hline $\mathrm{O}$ & -1.0540 & 4.4880 & 0.2880 & $\mathrm{O}$ & -0.7660 & 4.4640 & -0.7760 \\
\hline $\mathrm{O}$ & 4.1210 & -1.9530 & 2.3630 & $\mathrm{O}$ & 4.0530 & -1.3720 & 2.2790 \\
\hline $\mathrm{O}$ & 3.5710 & -0.3590 & 0.0360 & $\mathrm{O}$ & 3.5810 & -0.4840 & -0.4120 \\
\hline $\mathrm{C}$ & 5.2850 & -1.2960 & 1.8580 & $\mathrm{C}$ & 4.3290 & -1.8020 & 3.6150 \\
\hline $\mathrm{C}$ & 3.3090 & 0.5510 & -1.0380 & $\mathrm{C}$ & 4.9240 & -0.2550 & 0.0220 \\
\hline $\mathrm{C}$ & -0.8870 & 4.9610 & 1.6290 & $\mathrm{C}$ & 0.1010 & 5.2530 & -1.5980 \\
\hline $\mathrm{C}$ & -1.3100 & 5.0800 & -2.2670 & $\mathrm{C}$ & -2.4580 & 4.3810 & 1.2420 \\
\hline $\mathrm{C}$ & -3.1410 & -1.2760 & -1.4080 & $\mathrm{C}$ & -3.0300 & -1.4110 & -1.8300 \\
\hline
\end{tabular}




\begin{tabular}{|l|l|l|l|l|l|l|l|}
\hline O & -0.8550 & -1.3400 & -2.1800 & O & -0.7390 & -1.7760 & -2.4920 \\
\hline C & -0.9170 & -2.6600 & -2.7090 & C & -1.1180 & -1.5490 & -3.8470 \\
\hline H & 1.0560 & -0.5550 & -0.7960 & H & 1.2570 & -0.8530 & -1.2470 \\
\hline H & 2.1020 & -3.1750 & 3.2990 & H & 1.8550 & -2.4130 & 3.4350 \\
\hline H & -1.2820 & -4.7650 & 1.1370 & H & -1.2910 & -4.4470 & 1.3350 \\
\hline H & -2.5140 & -4.1170 & 2.1960 & H & -2.6180 & -3.6590 & 2.1620 \\
\hline H & -0.1200 & -4.1860 & 3.1620 & H & -0.3390 & -3.4470 & 3.3310 \\
\hline H & -0.9660 & -2.6650 & 3.3210 & H & -1.1850 & -1.9370 & 3.0770 \\
\hline H & -1.1700 & 2.4680 & 2.0380 & H & 0.4420 & 2.9530 & -2.6220 \\
\hline H & -1.4540 & 0.1540 & 1.4810 & H & 0.1480 & 0.5920 & -2.9050 \\
\hline H & -1.7000 & 1.6970 & -3.6930 & H & -3.2740 & 0.7740 & 1.2720 \\
\hline H & 5.5060 & -1.6430 & 0.8470 & H & 3.7080 & -1.2530 & 4.3240 \\
\hline H & 6.1300 & -1.5440 & 2.5000 & H & 5.3770 & -1.5990 & 3.8370 \\
\hline H & 5.1460 & -0.2140 & 1.8750 & H & 4.1540 & -2.8760 & 3.7090 \\
\hline H & 2.9480 & 0.0080 & -1.9130 & H & 4.9350 & 0.4210 & 0.8790 \\
\hline H & 4.2390 & 1.0540 & -1.3030 & H & 5.4760 & 0.2140 & -0.7920 \\
\hline H & 2.5800 & 1.3010 & -0.7270 & H & 5.4090 & -1.2020 & 0.2660 \\
\hline H & -1.7700 & 4.7250 & 2.2260 & H & 1.1310 & 4.9040 & -1.5050 \\
\hline H & -0.7640 & 6.0440 & 1.6040 & H & 0.0530 & 6.2890 & -1.2610 \\
\hline H & 0.0050 & 4.5200 & 2.0780 & H & -0.2230 & 5.2100 & -2.6400 \\
\hline H & -2.1400 & 5.4570 & -1.6670 & H & -1.4480 & 4.6720 & 1.5350 \\
\hline H & -1.3770 & 5.5210 & -3.2620 & H & -3.1220 & 4.5540 & 2.0890 \\
\hline H & -0.3510 & 5.3640 & -1.8320 & H & -2.8120 & 4.9830 & 0.4030 \\
\hline H & -3.8140 & -0.9790 & -0.6020 & H & -3.7180 & -1.1580 & -1.0230 \\
\hline H & -3.3690 & -2.3200 & -1.6240 & H & -3.2190 & -2.4570 & -2.0770 \\
\hline H & -3.4300 & -0.7050 & -2.2920 & H & -3.3430 & -0.8170 & -2.6900 \\
\hline H & -1.9050 & -2.8750 & -3.1130 & H & -1.3920 & -0.5130 & -4.0420 \\
\hline H & -0.6500 & -3.4070 & -1.9620 & H & -1.9600 & -2.1910 & -4.1070 \\
\hline H & -0.2020 & -2.7300 & -3.5200 & H & -0.2790 & -1.8170 & -4.4900 \\
\hline & & & & & & & \\
\hline
\end{tabular}

Table S7. Cartesian Coordinates, Relative Energies, and Equilibrium Populations of Low-energy Conformers (>1\%) of $9 R *_{-6}$.

\begin{tabular}{|l|l|l|l|l|l|l|l|}
\hline \multicolumn{3}{|c|}{ Conformer 1} & \multicolumn{5}{c|}{ Conformer 2} \\
\hline $\mathrm{C}$ & -3.7791 & 2.8626 & 0.2248 & $\mathrm{C}$ & -3.7628 & 2.9132 & 0.2061 \\
\hline $\mathrm{C}$ & -3.8355 & 1.4545 & 0.0356 & $\mathrm{C}$ & -3.8711 & 1.5043 & 0.0733 \\
\hline $\mathrm{C}$ & -2.6412 & 0.7534 & -0.1931 & $\mathrm{C}$ & -2.6968 & 0.7320 & -0.0904 \\
\hline $\mathrm{C}$ & -1.3562 & 1.3572 & -0.1819 & $\mathrm{C}$ & -1.4067 & 1.3186 & -0.1491 \\
\hline
\end{tabular}




\begin{tabular}{|c|c|c|c|c|c|c|c|}
\hline $\mathrm{C}$ & -1.3355 & 2.7626 & -0.0051 & $\mathrm{C}$ & -1.3379 & 2.7312 & -0.0151 \\
\hline $\mathrm{C}$ & -2.5063 & 3.4841 & 0.2028 & $\mathrm{C}$ & -2.4958 & 3.4944 & 0.1718 \\
\hline $\mathrm{C}$ & -0.1390 & 0.6559 & -0.3810 & $\mathrm{C}$ & -0.1662 & 0.6275 & -0.3719 \\
\hline $\mathrm{N}$ & 1.0254 & 1.4030 & -0.5204 & $\mathrm{~N}$ & 0.9932 & 1.3746 & -0.5206 \\
\hline $\mathrm{C}$ & 1.0372 & 2.7658 & -0.3720 & $\mathrm{C}$ & 1.0214 & 2.7344 & -0.3759 \\
\hline $\mathrm{C}$ & -0.1209 & 3.4688 & -0.0920 & $\mathrm{C}$ & -0.1386 & 3.4370 & -0.0978 \\
\hline $\mathrm{C}$ & -0.0568 & -0.8617 & -0.4675 & $\mathrm{C}$ & -0.0774 & -0.8816 & -0.4682 \\
\hline $\mathrm{C}$ & 1.2958 & -1.3845 & -0.1040 & $\mathrm{C}$ & 1.2825 & -1.3949 & -0.1032 \\
\hline $\mathrm{C}$ & 2.4402 & -0.5737 & -0.2883 & $\mathrm{C}$ & 2.4239 & -0.5807 & -0.2864 \\
\hline $\mathrm{C}$ & 2.2691 & 0.7777 & -0.8701 & $\mathrm{C}$ & 2.2487 & 0.7643 & -0.8717 \\
\hline $\mathrm{C}$ & 1.4684 & -2.6944 & 0.4031 & $\mathrm{C}$ & 1.4618 & -2.7012 & 0.4076 \\
\hline $\mathrm{C}$ & 2.7316 & -3.1533 & 0.7833 & $\mathrm{C}$ & 2.7276 & -3.1547 & 0.7845 \\
\hline $\mathrm{C}$ & 3.8749 & -2.3511 & 0.5925 & $\mathrm{C}$ & 3.8681 & -2.3480 & 0.5946 \\
\hline $\mathrm{C}$ & 3.7284 & -1.0880 & -0.0316 & $\mathrm{C}$ & 3.7171 & -1.0843 & -0.0248 \\
\hline $\mathrm{O}$ & 4.7489 & -0.3429 & -0.5394 & $\mathrm{O}$ & 4.7363 & -0.3328 & -0.5270 \\
\hline $\mathrm{O}$ & 5.1075 & -2.7808 & 0.9764 & $\mathrm{O}$ & 5.1023 & -2.7748 & 0.9771 \\
\hline $\mathrm{O}$ & -4.8949 & 3.6385 & & $\mathrm{O}$ & -4.8469 & & \\
\hline $\mathrm{O}$ & -5.0251 & 0.7657 & -0.0208 & $\mathrm{O}$ & -5.0316 & 0.8040 & -0.0471 \\
\hline $\mathrm{C}$ & -5.0135 & 5.0808 & 0.2600 & $\mathrm{C}$ & -4.9476 & 5.1650 & 0.2524 \\
\hline $\mathrm{C}$ & -5.2227 & -0.6035 & -0.4462 & $\mathrm{C}$ & -6.3256 & 1.3032 & -0.3916 \\
\hline $\mathrm{C}$ & 6.1091 & -0.6820 & -0.7589 & $\mathrm{C}$ & 6.0936 & -0.6790 & -0.7574 \\
\hline $\mathrm{C}$ & 5.4604 & -4.0194 & 1.5964 & $\mathrm{C}$ & 5.4535 & -4.0149 & 1.5940 \\
\hline $\mathrm{O}$ & -0.9714 & -1.4881 & & $\mathrm{O}$ & & -1.5131 & 372 \\
\hline $\mathrm{C}$ & -0.4110 & -1.3373 & & $\mathrm{C}$ & -0.42 & .3443 & \\
\hline $\mathrm{H}$ & -2.7043 & -0.2294 & -0.4022 & $\mathrm{H}$ & -2.8373 & -0.2676 & -0.2395 \\
\hline $\mathrm{H}$ & -2.4312 & 4.4899 & 0.3238 & $\mathrm{H}$ & -2.3922 & 4.4982 & 0.2785 \\
\hline $\mathrm{H}$ & 1.9002 & 3.3154 & -0.4671 & $\mathrm{H}$ & 1.8799 & 3.2867 & -0.4674 \\
\hline $\mathrm{H}$ & -0.0862 & 4.4866 & 0.0244 & $\mathrm{H}$ & -0.0905 & 4.4552 & 0.0186 \\
\hline $\mathrm{H}$ & & 1.4066 & & $\mathrm{H}$ & 3.0916 & .3971 & -0.5670 \\
\hline $\mathrm{H}$ & 2.3104 & 0.6819 & -1.9586 & $\mathrm{H}$ & 2.2962 & 0.6729 & -1.9598 \\
\hline $\mathrm{H}$ & 0.6730 & -3.3304 & & $\mathrm{H}$ & & -3.3388 & 0.5161 \\
\hline $\mathrm{H}$ & 2.8097 & -4.0923 & 1.1749 & $\mathrm{H}$ & 2.8093 & -4.0934 & 1.1746 \\
\hline $\mathrm{H}$ & -4.2012 & 5.6218 & 0.7472 & $\mathrm{H}$ & -4.1181 & 5.6999 & 0.7159 \\
\hline $\mathrm{H}$ & -5.9352 & 5.4109 & 0.7304 & $\mathrm{H}$ & -5.8588 & 5.4990 & 0.7403 \\
\hline $\mathrm{H}$ & -5.0306 & & & $\mathrm{H}$ & & & -0.8063 \\
\hline $\mathrm{H}$ & -6.2620 & -0.9044 & -0.3367 & $\mathrm{H}$ & -7.0719 & 0.5204 & -0.2759 \\
\hline $\mathrm{H}$ & -4.6071 & -1.3090 & 0.1201 & $\mathrm{H}$ & -6.2777 & 1.5943 & -1.4424 \\
\hline $\mathrm{H}$ & -4.9507 & -0.6731 & -1.5030 & $\mathrm{H}$ & -6.6304 & 2.1564 & 0.2098 \\
\hline $\mathrm{H}$ & 6.5837 & 0.1346 & -1.3012 & $\mathrm{H}$ & 6.5687 & 0.1332 & -1.3057 \\
\hline $\mathrm{H}$ & 6.1276 & -1.5776 & -1.3817 & $\mathrm{H}$ & 6.0981 & -1.5744 & -1.3808 \\
\hline $\mathrm{H}$ & 6.6400 & -0.8599 & 0.1755 & $\mathrm{H}$ & 6.6342 & -0.8619 & 0.1705 \\
\hline $\mathrm{H}$ & 5.1975 & -4.8468 & 0.9349 & $\mathrm{H}$ & 5.1885 & -4.8399 & 0.9303 \\
\hline $\mathrm{H}$ & 4.9316 & -4.1251 & & $\mathrm{H}$ & 4.9227 & -4.1201 & 2.5423 \\
\hline $\mathrm{H}$ & 6.5326 & -4.0380 & 1.7818 & $\mathrm{H}$ & 6.5252 & -4.0371 & 1.7807 \\
\hline $\mathrm{H}$ & -0.5418 & -1.4980 & 1.3293 & $\mathrm{H}$ & -0.5604 & -1.5222 & 1.3199 \\
\hline
\end{tabular}




\begin{tabular}{|l|l|l|l|l|l|l|l|}
\hline $\mathrm{H}$ & -0.3533 & -2.4261 & -1.9864 & $\mathrm{H}$ & -0.3650 & -2.4322 & -1.9989 \\
\hline $\mathrm{H}$ & 0.2768 & -0.9095 & -2.6437 & $\mathrm{H}$ & 0.2626 & -0.9093 & -2.6435 \\
\hline $\mathrm{H}$ & -1.4229 & -1.0443 & -2.1920 & $\mathrm{H}$ & -1.4399 & -1.0472 & -2.1875 \\
\hline
\end{tabular}

Table S8. Extracted heats and weighting factors of the optimized conformers of $1 \mathrm{~A}\left(1 R^{*}, 9 S *-1\right)$ and $1 \mathrm{~B}\left(1 S^{*}, 9 S^{*-1}\right)$ at B3LYP/6-311+G(d, p) level in MeOH with PCM model.

\begin{tabular}{|c|c|c|c|}
\hline & & \multicolumn{2}{|c|}{ B3LYP/6-311+G(d,p) } \\
\hline \multirow{4}{*}{$\mathbf{1 A}$} & Conformer & Extracted heats & $\begin{array}{c}\text { Boltzmann-calculated } \\
\text { contribution (\%) }\end{array}$ \\
\cline { 2 - 4 } & 1 & -1435.061686 & 0.48 \\
\cline { 2 - 4 } & 2 & -1435.0644005 & 8.60 \\
\cline { 2 - 4 } & 3 & -1435.0664575 & 76.10 \\
\cline { 2 - 4 } & 4 & -1435.0609194 & 0.21 \\
\hline \multirow{3}{*}{$\mathbf{1 B}$} & 5 & -1435.0649 & 14.60 \\
\cline { 2 - 4 } & 1 & -1434.7554837 & 33.29 \\
\cline { 2 - 4 } & 2 & -1434.7507031 & 42.83 \\
\hline
\end{tabular}

Table S9. Extracted heats and weighting factors of the optimized conformers of $9 R *-2$ at B3LYP/6-311+G (d, p) level in MeOH with PCM model.

\begin{tabular}{|c|c|c|c|}
\hline & & \multicolumn{2}{|c|}{ B3LYP/6-311+G(d,p) } \\
\hline & Conformer & Extracted heats & $\begin{array}{c}\text { Boltzmann-calculated } \\
\text { contribution (\%) }\end{array}$ \\
\hline \multirow{4}{*}{$\mathbf{9} \boldsymbol{R}^{*-2}$} & 1 & -1357.4262588 & 0.28 \\
\cline { 2 - 4 } & 2 & -1357.4316098 & 98.18 \\
\cline { 2 - 4 } & 3 & -1357.4276297 & 1.11 \\
\hline
\end{tabular}

Table S10. Extracted heats and weighting factors of the optimized conformers of $9 S *-3$ at B3LYP/6-311+G (d, p) level in MeOH with PCM model.

\begin{tabular}{|c|c|c|c|}
\hline & & \multicolumn{2}{|c|}{ B3LYP/6-311+G(d,p) } \\
\hline \multirow{4}{*}{} & Conformer & Extracted heats & $\begin{array}{c}\text { Boltzmann-calculated } \\
\text { contribution (\%) }\end{array}$ \\
\hline \multirow{4}{*}{$\mathbf{9} \mathbf{S}^{*-3}$} & 1 & -1245.2639122 & 0.33 \\
\cline { 2 - 4 } & 2 & -1245.2690985 & 80.46 \\
\cline { 2 - 4 } & 3 & -1245.2646084 & 0.69 \\
\cline { 2 - 4 } & 4 & -1245.2670605 & 9.27 \\
\cline { 2 - 4 } & 5 & -1245.2670571 & 9.24 \\
\hline
\end{tabular}


Table S11. Extracted heats and weighting factors of the optimized conformers of $9 S *-4$ at B3LYP/6-311+G (d, p) level in MeOH with PCM model.

\begin{tabular}{|c|c|c|c|}
\hline & & \multicolumn{2}{|c|}{ B3LYP/6-311+G(d,p) } \\
\hline \multirow{4}{*}{} & Conformer & Extracted heats & $\begin{array}{c}\text { Boltzmann-calculated } \\
\text { contribution (\%) }\end{array}$ \\
\hline \multirow{4}{*}{$\mathbf{9} *-4$} & 1 & -1246.4571067 & 7.60 \\
\cline { 2 - 4 } & 2 & -1246.4582582 & 25.75 \\
\cline { 2 - 4 } & 3 & -1246.4584715 & 32.28 \\
\cline { 2 - 4 } & 4 & -1246.4568967 & 6.08 \\
\cline { 2 - 4 } & 5 & -1246.4583474 & 28.30 \\
\hline
\end{tabular}

Table S12. Extracted heats and weighting factors of the optimized conformers of $9 R^{*-5}$ at B3LYP/6-311+G (d, p) level in MeOH with PCM model.

\begin{tabular}{|c|c|c|c|}
\hline & & \multicolumn{2}{|c|}{ B3LYP/6-311+G(d,p) } \\
\hline & Conformer & Extracted heats & $\begin{array}{c}\text { Boltzmann-calculated } \\
\text { contribution (\%) }\end{array}$ \\
\hline \multirow{2}{*}{$\mathbf{9} \boldsymbol{R}^{*-5}$} & 1 & -1360.9963964 & 15.51 \\
\cline { 2 - 4 } & 2 & -1360.997971 & 84.49 \\
\hline
\end{tabular}

Table S13. Extracted heats and weighting factors of the optimized conformers of $9 R *-6$ at B3LYP/6-311+G (d, p) level in MeOH with PCM model.

\begin{tabular}{|c|c|c|c|}
\hline & & \multicolumn{2}{|c|}{ B3LYP/6-311+G(d,p) } \\
\hline & Conformer & Extracted heats & $\begin{array}{c}\text { Boltzmann-calculated } \\
\text { contribution (\%) }\end{array}$ \\
\hline \multirow{2}{*}{$\mathbf{9 R} * \mathbf{- 6}$} & 1 & -1283.9324334 & 96.62 \\
\cline { 2 - 4 } & 2 & -1283.9293728 & 3.38 \\
\hline
\end{tabular}

Table S14. Calculated ${ }^{13} \mathrm{C}$ NMR chemical shifts (ppm) and DP4+ for $1 \mathrm{~A}\left(1 R^{*}, 9 S^{*-1}\right)$ and $1 \mathrm{~B}\left(1 S^{*}\right.$, $\left.9 S^{*}-1\right)$

\begin{tabular}{|c|c|c|c|c|c|c|c|c|c|c|}
\hline \multirow{3}{*}{ No. } & \multicolumn{5}{|c|}{ DP4+ } & $0.00 \%$ & \multicolumn{3}{|c|}{ DP4+ } & $100.00 \%$ \\
\hline & \multicolumn{5}{|c|}{ Calculated } & Averaged $^{a}$ & \multicolumn{3}{|c|}{ Calculated } & Averaged $^{a}$ \\
\hline & 1A-1 & 1A-2 & $1 \mathrm{~A}-3$ & $1 A-4$ & $1 A-5$ & $\mathbf{1 A}$ & 1B-1 & 1B-2 & $1 B-3$ & $1 B$ \\
\hline 1 & 112.9 & 114.8 & 113.9 & 113.8 & 115.0 & 114.1 & 115.2 & 115.7 & 115.2 & 115.4 \\
\hline 3 & 51.4 & 54.2 & 55.6 & 51.9 & 55.4 & 55.4 & 47.2 & 50.7 & 55.4 & 50.7 \\
\hline 4 & 38.2 & 40.3 & 40.1 & 37.3 & 40.4 & 40.1 & 39.1 & 38.7 & 39.3 & 39.0 \\
\hline $4 a$ & 140.4 & 139.1 & 136.0 & 135.8 & 135.9 & 136.3 & 134.7 & 141.4 & 137.7 & 138.3 \\
\hline
\end{tabular}




\begin{tabular}{|c|c|c|c|c|c|c|c|c|c|c|}
\hline 5 & 114.8 & 114.5 & 126.1 & 126.2 & 113.8 & 123.0 & 113.7 & 115.3 & 126.7 & 117.5 \\
\hline 6 & 158.4 & 158.4 & 153.3 & 153.5 & 153.9 & 153.9 & 154.1 & 159.0 & 153.1 & 156.0 \\
\hline 7 & 151.6 & 152.0 & 156.8 & 156.7 & 152.7 & 155.6 & 151.2 & 151.6 & 156.9 & 152.7 \\
\hline 8 & 124.6 & 127.0 & 114.3 & 113.3 & 113.2 & 115.5 & 114.7 & 123.7 & 112.5 & 118.0 \\
\hline $8 \mathrm{a}$ & 132.9 & 134.1 & 138.1 & 137.2 & 133.0 & 136.9 & 132.9 & 131.4 & 136.7 & 133.2 \\
\hline 9 & 98.3 & 96.2 & 98.6 & 98.2 & 98.1 & 98.2 & 96.3 & 97.5 & 98.1 & 97.3 \\
\hline 10 & 35.2 & 34.3 & 33.4 & 35.0 & 33.7 & 33.5 & 37.8 & 37.1 & 34.8 & 36.8 \\
\hline $1^{\prime}$ & 131.3 & 131.1 & 129.8 & 131.1 & 131.3 & 130.2 & 132.6 & 131.2 & 130.1 & 131.4 \\
\hline $2^{\prime}$ & 155.4 & 158.0 & 157.4 & 155.6 & 157.9 & 157.5 & 155.3 & 155.3 & 157.8 & 155.9 \\
\hline $3^{\prime}$ & 140.9 & 137.3 & 136.1 & 140.9 & 139.3 & 136.7 & 137.8 & 140.2 & 136.4 & 138.5 \\
\hline $4^{\prime}$ & 159.2 & 160.6 & 161.1 & 159.3 & 159.1 & 160.7 & 159.5 & 159.6 & 160.7 & 159.8 \\
\hline $5^{\prime}$ & 120.3 & 109.3 & 109.2 & 120.5 & 119.0 & 110.7 & 109.4 & 120.2 & 109.1 & 114.0 \\
\hline $6^{\prime}$ & 123.2 & 124.1 & 125.0 & 123.2 & 123.4 & 124.6 & 122.5 & 123.9 & 125.1 & 123.7 \\
\hline CHO & 164.0 & 168.5 & 170.3 & 163.9 & 170.3 & 170.1 & 163.7 & 165.7 & 167.3 & 165.4 \\
\hline OMe-6 & 61.8 & 61.7 & 66.7 & 66.7 & 62.2 & 65.5 & 62.0 & 61.9 & 66.9 & 63.1 \\
\hline OMe-7 & 66.8 & 67.3 & 61.8 & 61.8 & 61.9 & 62.4 & 61.5 & 66.9 & 61.7 & 63.9 \\
\hline OMe-3' & 67.2 & 67.2 & 67.5 & 67.1 & 66.3 & 67.3 & 66.3 & 66.5 & 67.9 & 66.8 \\
\hline OMe-4' & 68.1 & 62.5 & 62.5 & 68.1 & 67.8 & 63.3 & 62.6 & 68.3 & 62.4 & 65.0 \\
\hline
\end{tabular}

${ }^{a}$ Averaged accordingtotheBoltzmann-calculated contribution at B3LYP/6-311+G(d,p) level.

Figure S7. Correlation plots of experimental ${ }^{13} \mathrm{C}$ NMR chemical shifts versus corresponding calculated ${ }^{13} \mathrm{C}$ NMR chemical shifts for 1.
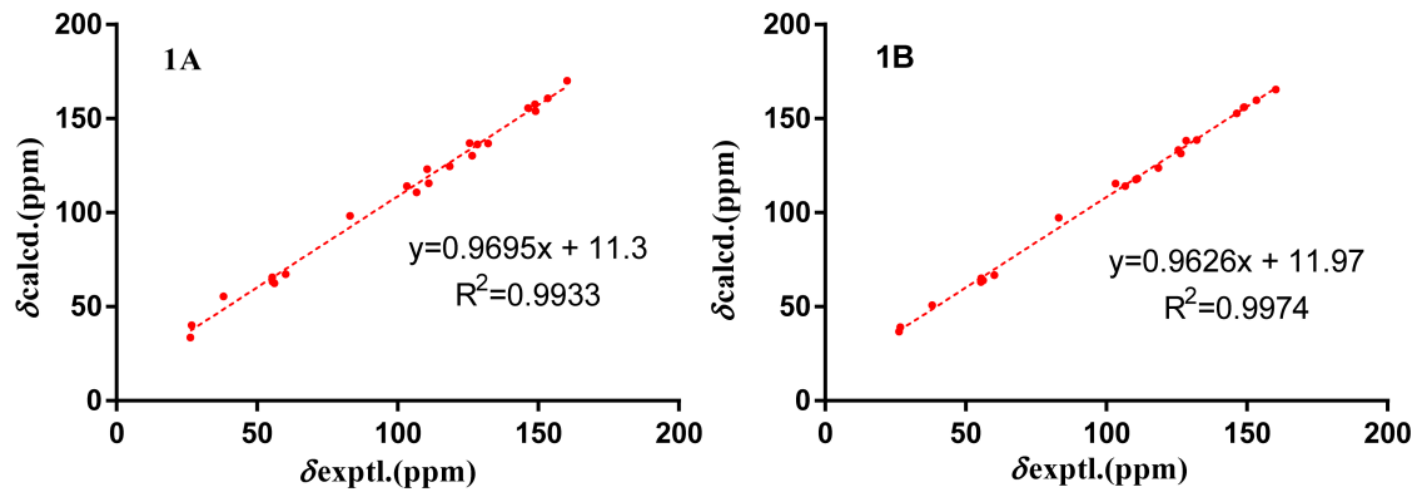


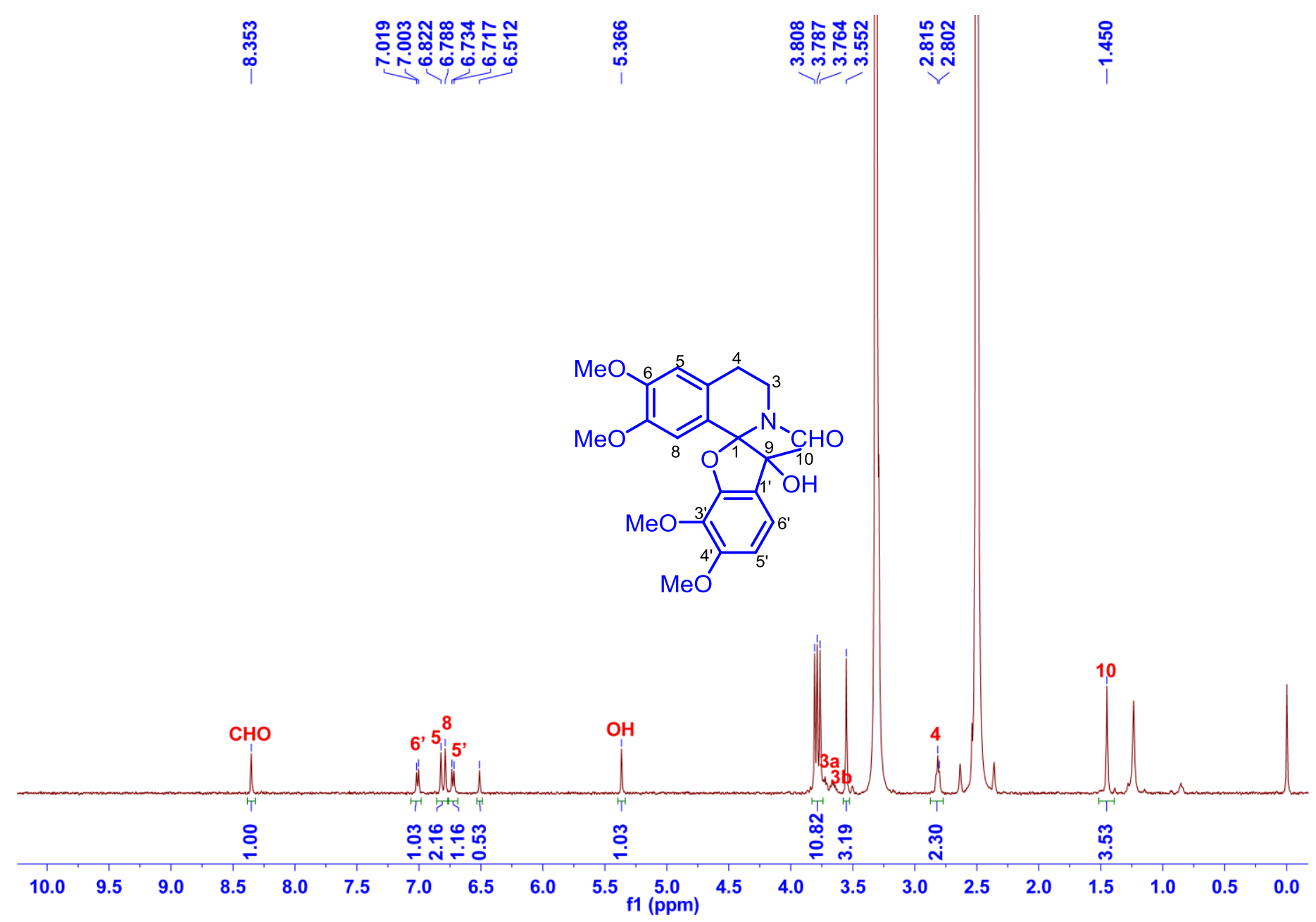

Figure S8. ${ }^{1} \mathrm{H}$ NMR spectrum of compound 1 in DMSO-d $(600 \mathrm{MHz})$ 


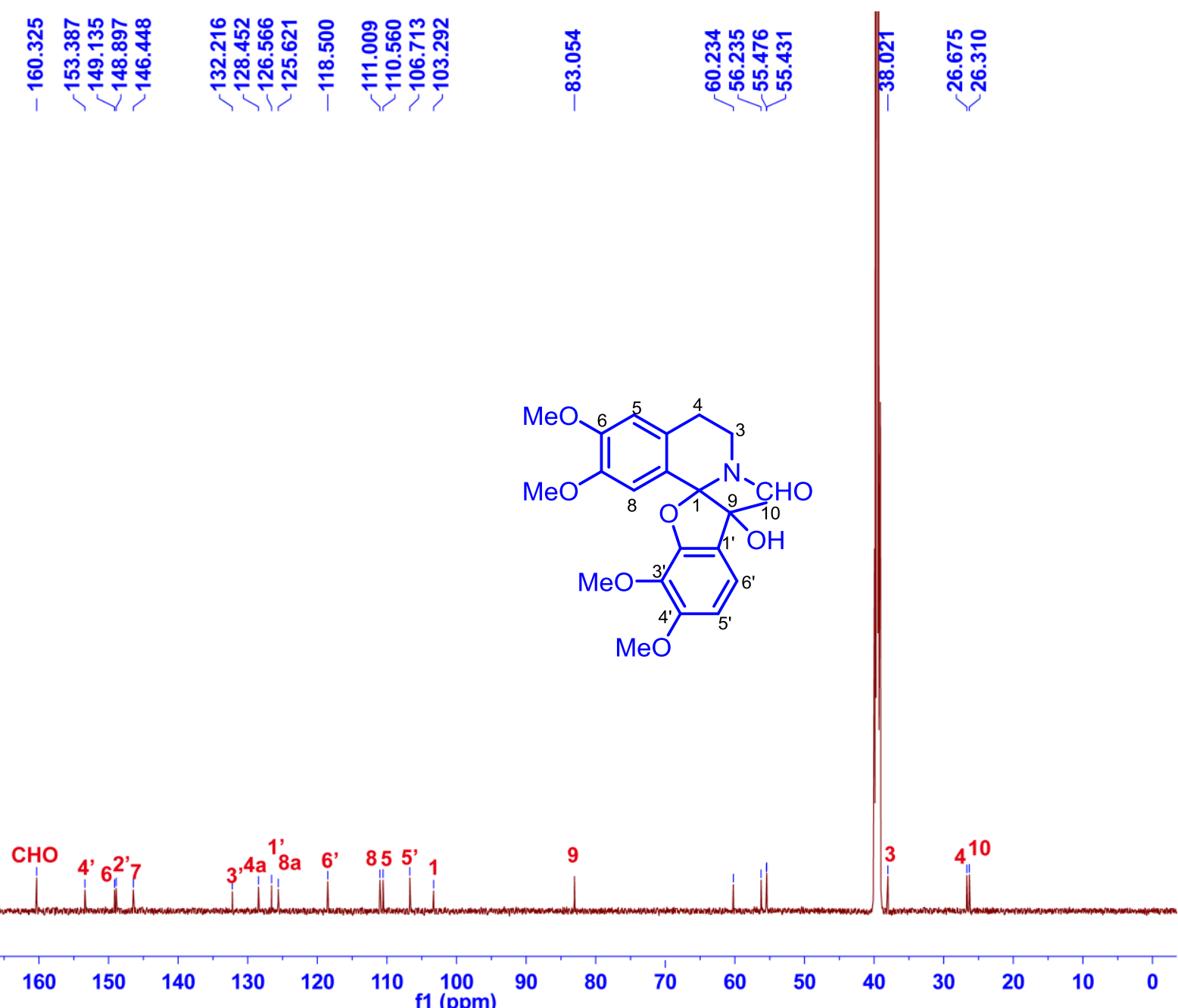

Figure S9. ${ }^{13} \mathrm{C}$ NMR spectrum of compound 1 in DMSO-d $6(150 \mathrm{MHz})$ 


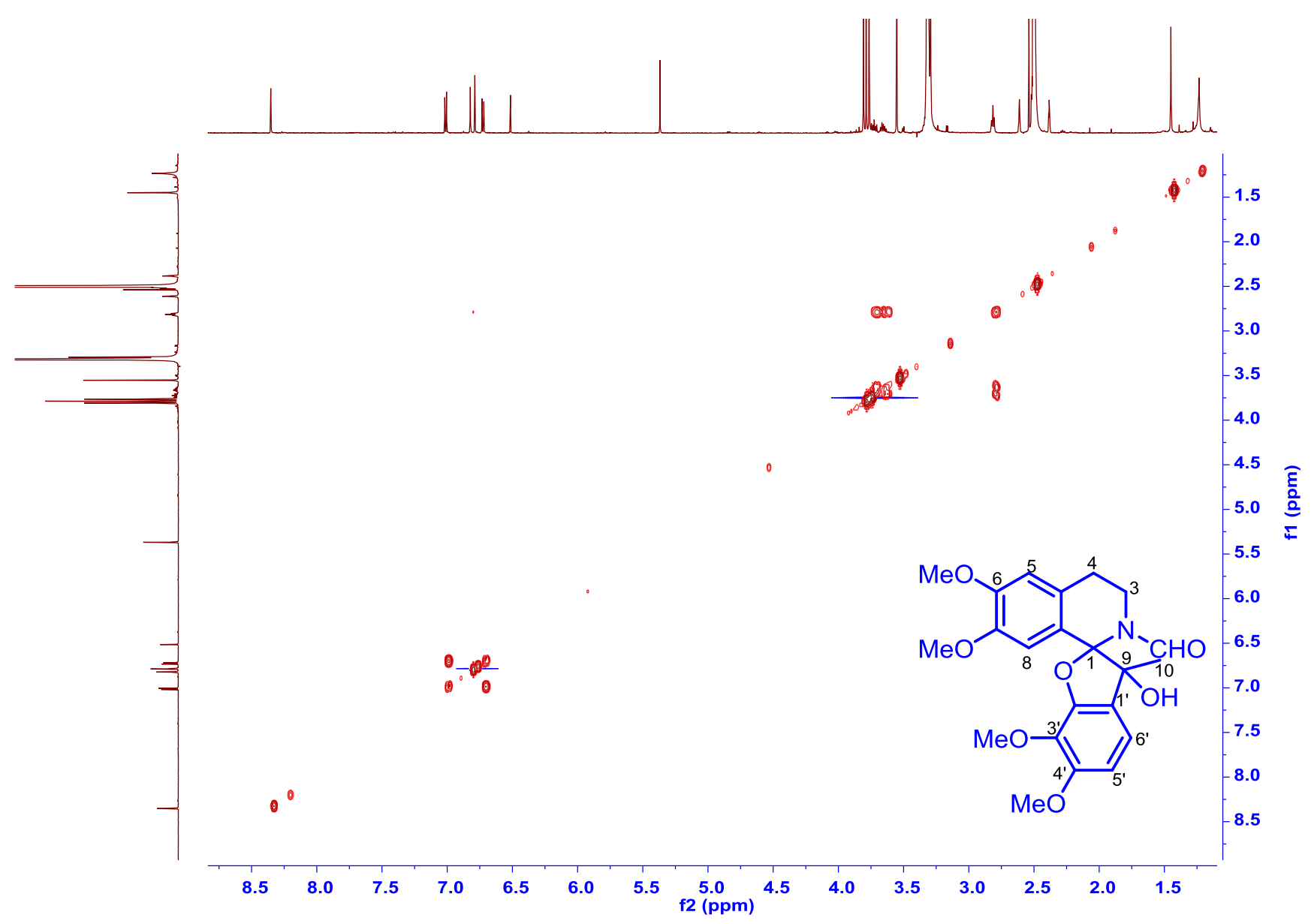

Figure S10. The ${ }^{1} \mathrm{H}-{ }^{1} \mathrm{H}$ COSY spectrum of compound 1 in DMSO- $d_{6}(600 \mathrm{MHz})$ 


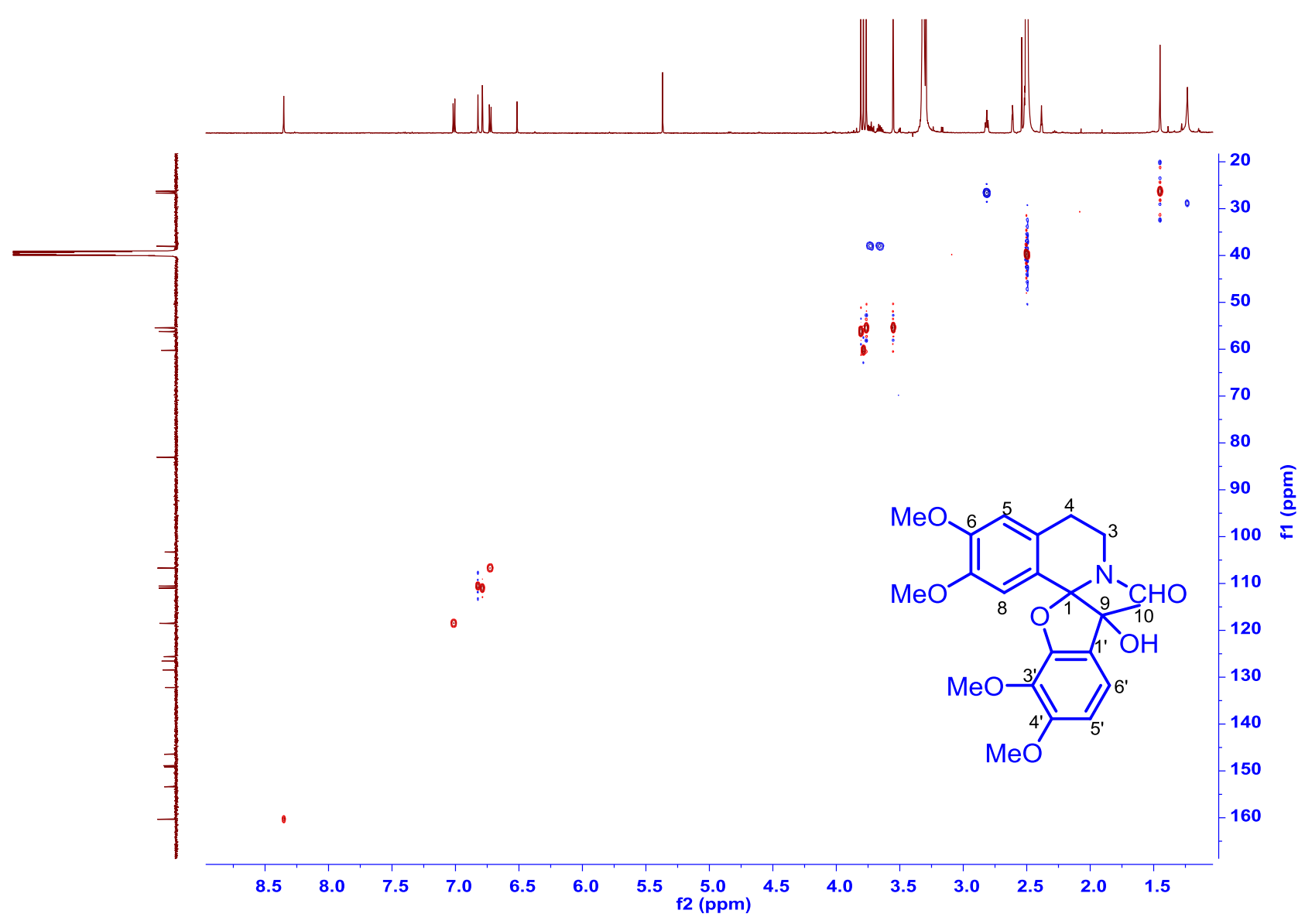

Figure S11. The HSQC spectrum of compound 1 in DMSO-d $6(600 \mathrm{MHz})$ 


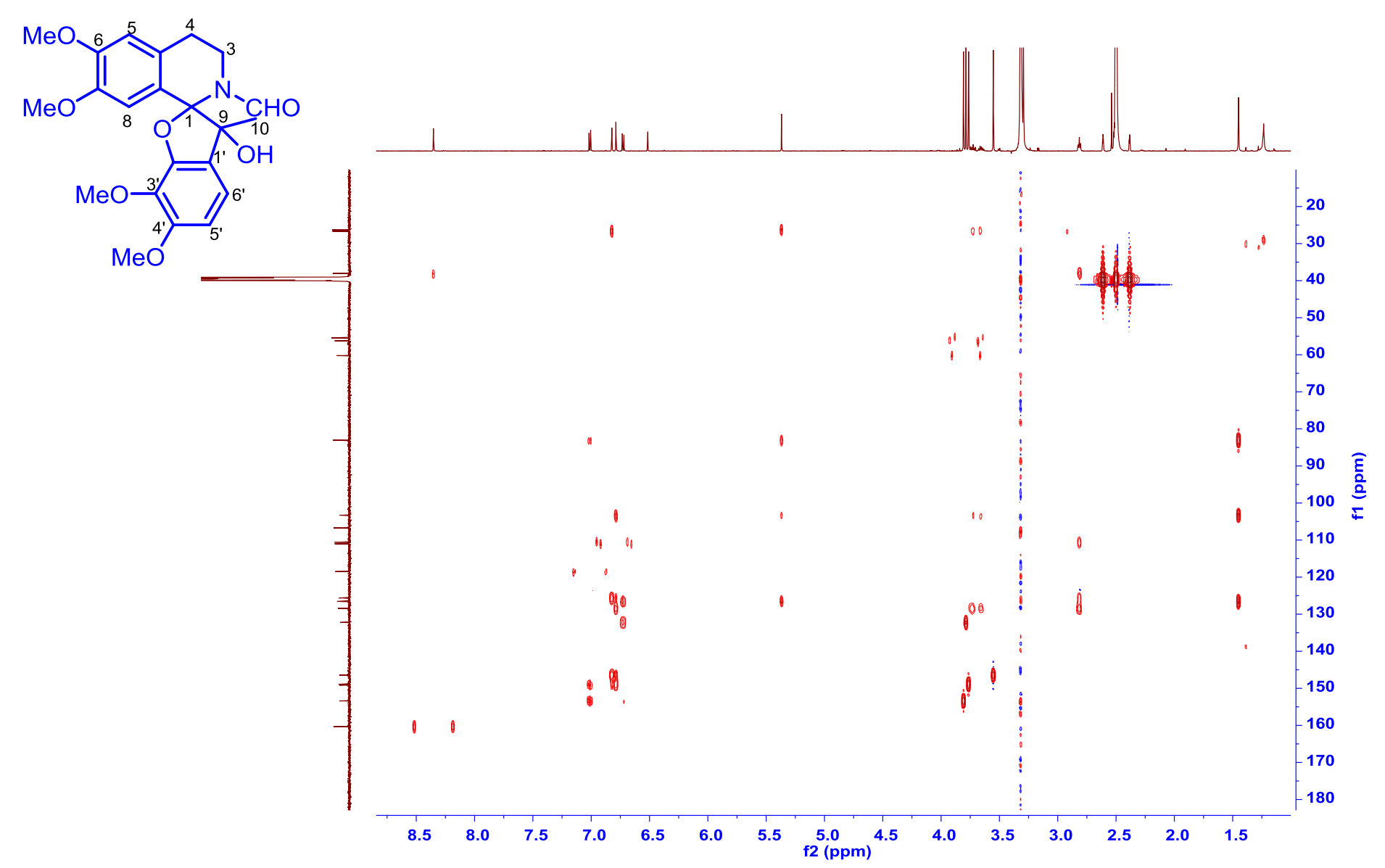

Figure S12. The HMBC spectrum of compound 1 in DMSO- $d_{6}(600 M H z)$ 


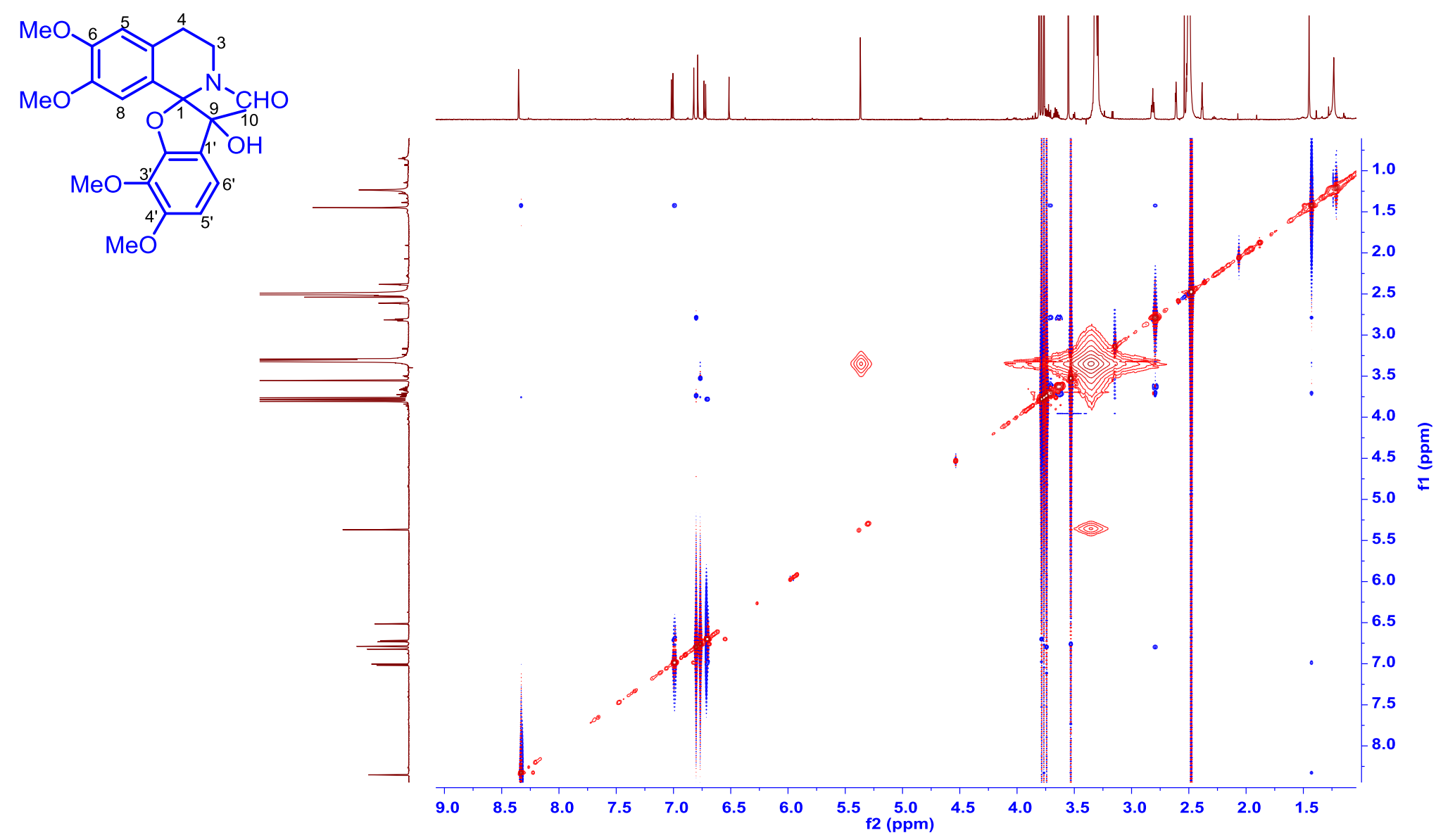

Figure S13. The NOESY spectrum of compound 1 in DMSO- $d_{6}(600 \mathrm{MHz})$ 


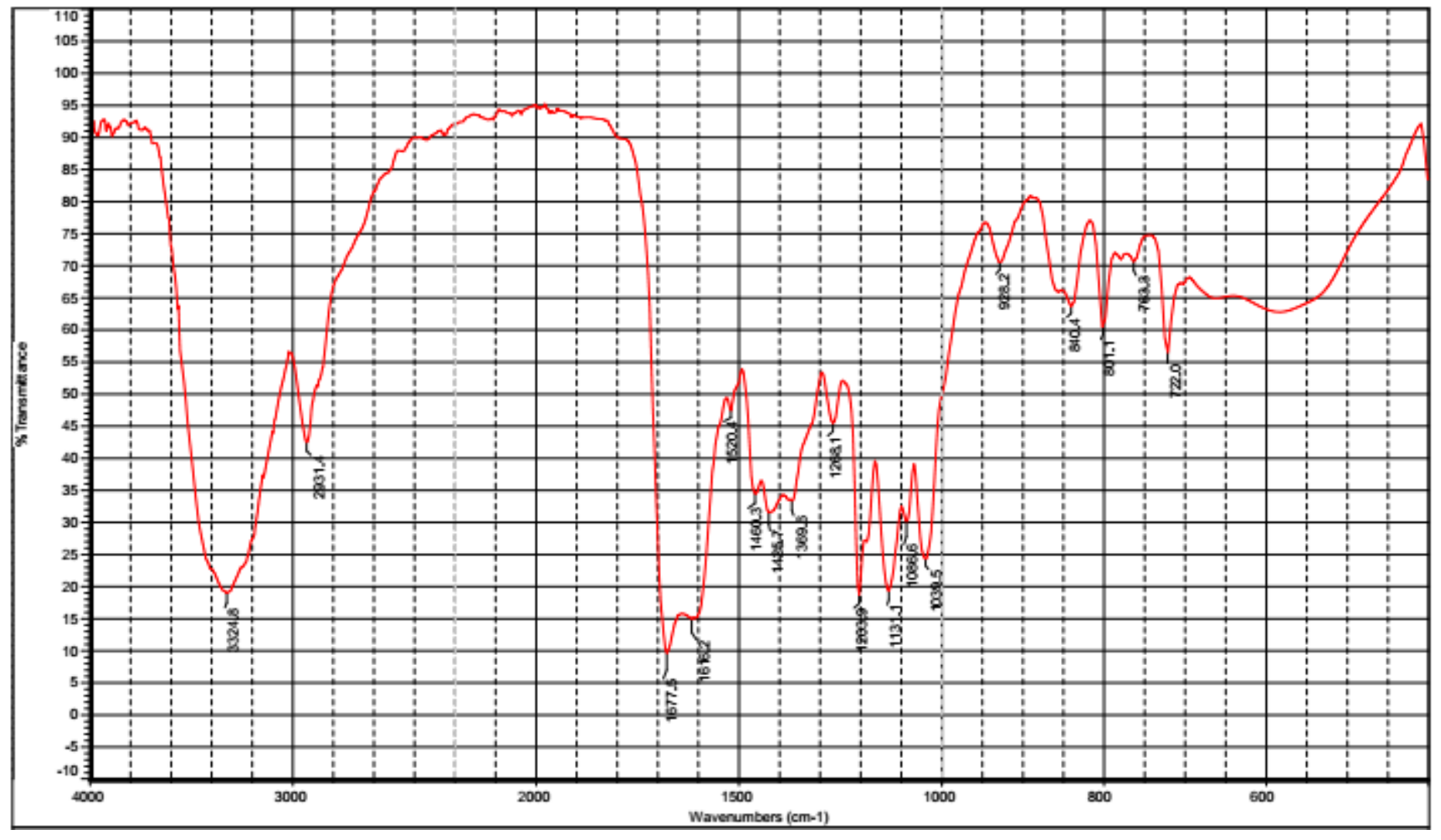

Figure S14. The IR spectrum of compound 1 


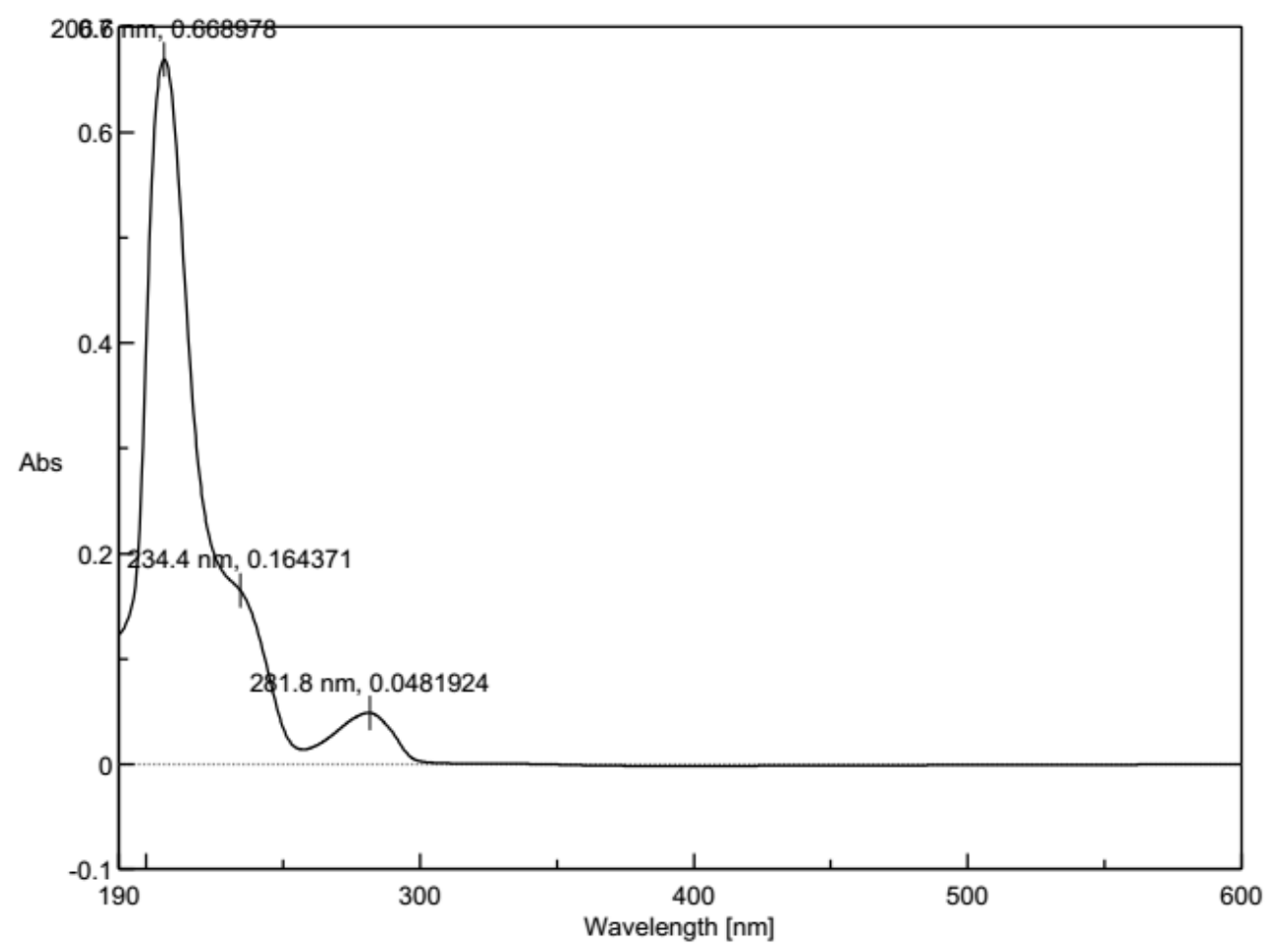

Figure S15. The UV spectrum of compound 1 in $\mathrm{MeOH}$

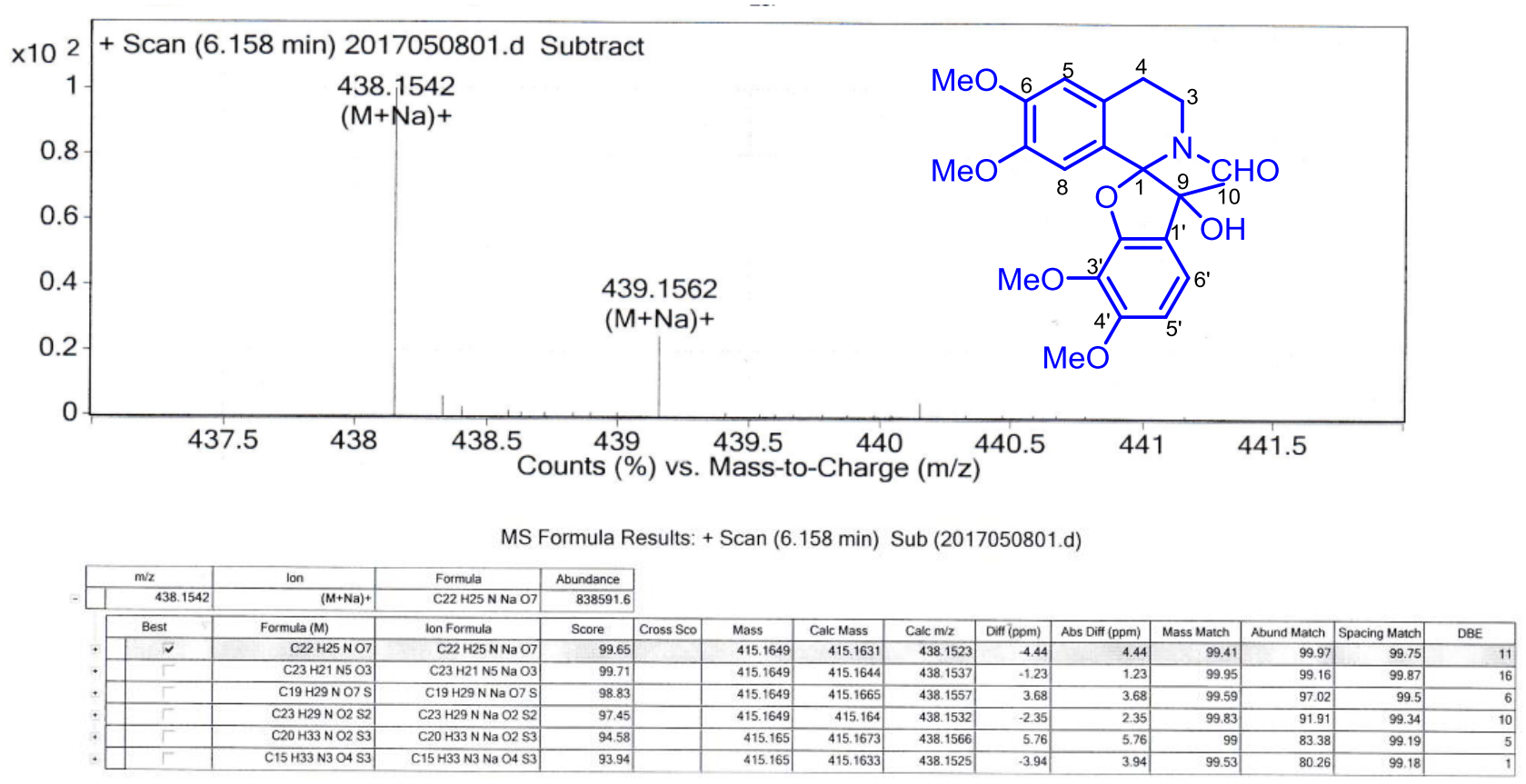

Figure S16. The HR-Mass spectrum of compound 1 in $\mathrm{MeOH}$ 


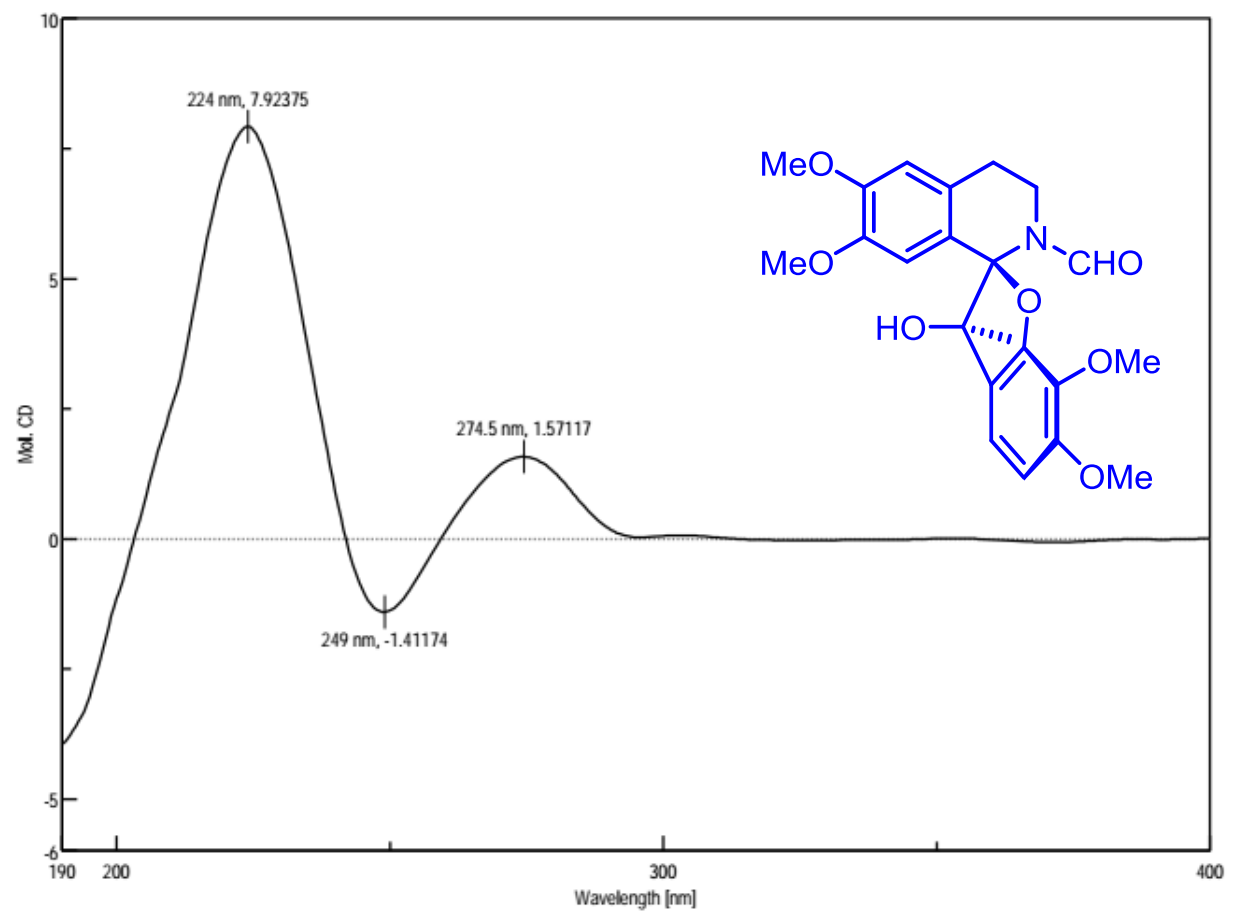

Figure S17. The CD spectrum of compound (+)-1 in $\mathrm{MeOH}$

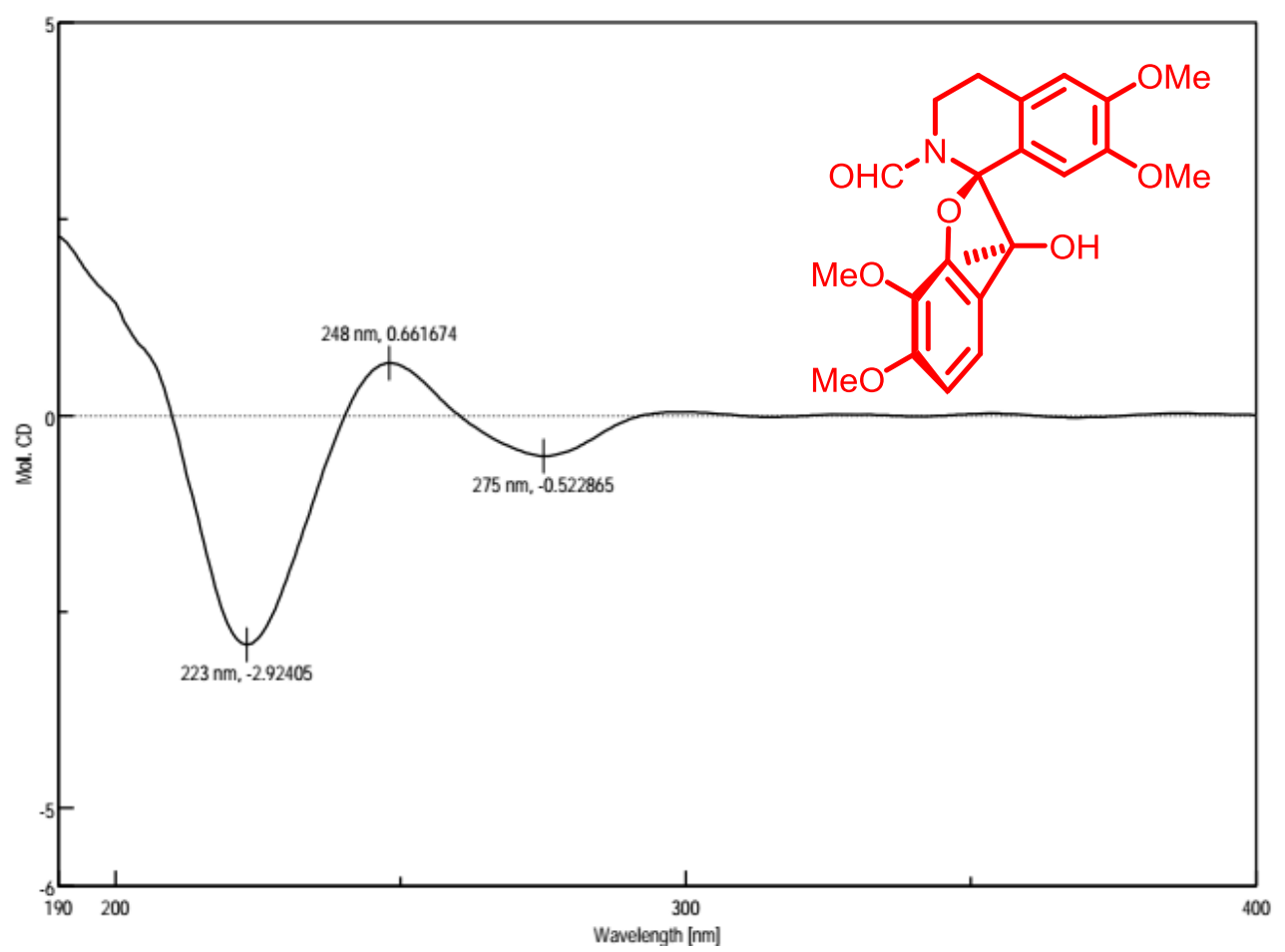

Figure S18. The CD spectrum of compound (-)-1 in $\mathrm{MeOH}$ 


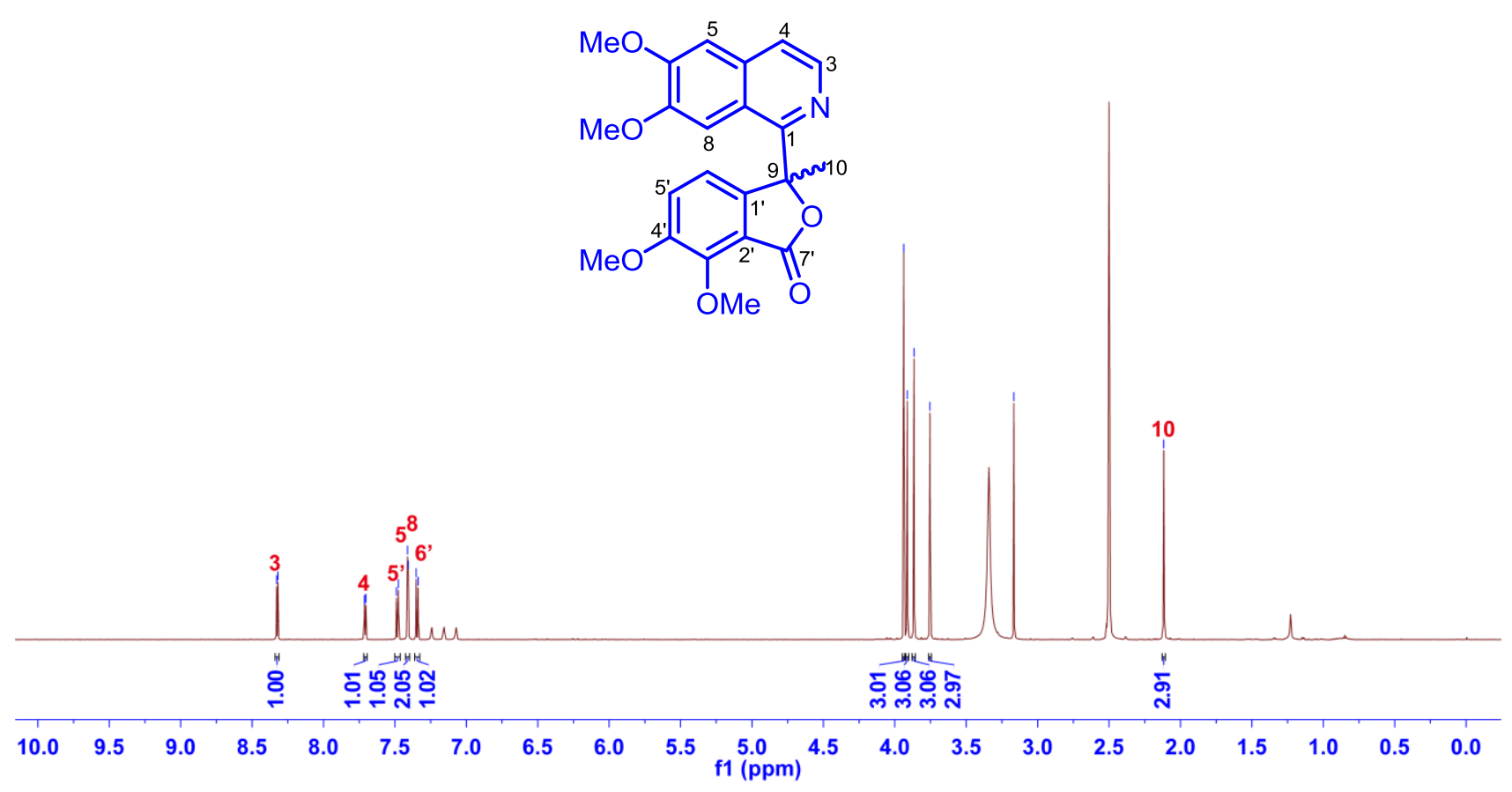

Figure S19. ${ }^{1} \mathrm{H}$ NMR spectrum of compound 2 in DMSO-d $(600 \mathrm{MHz})$ 

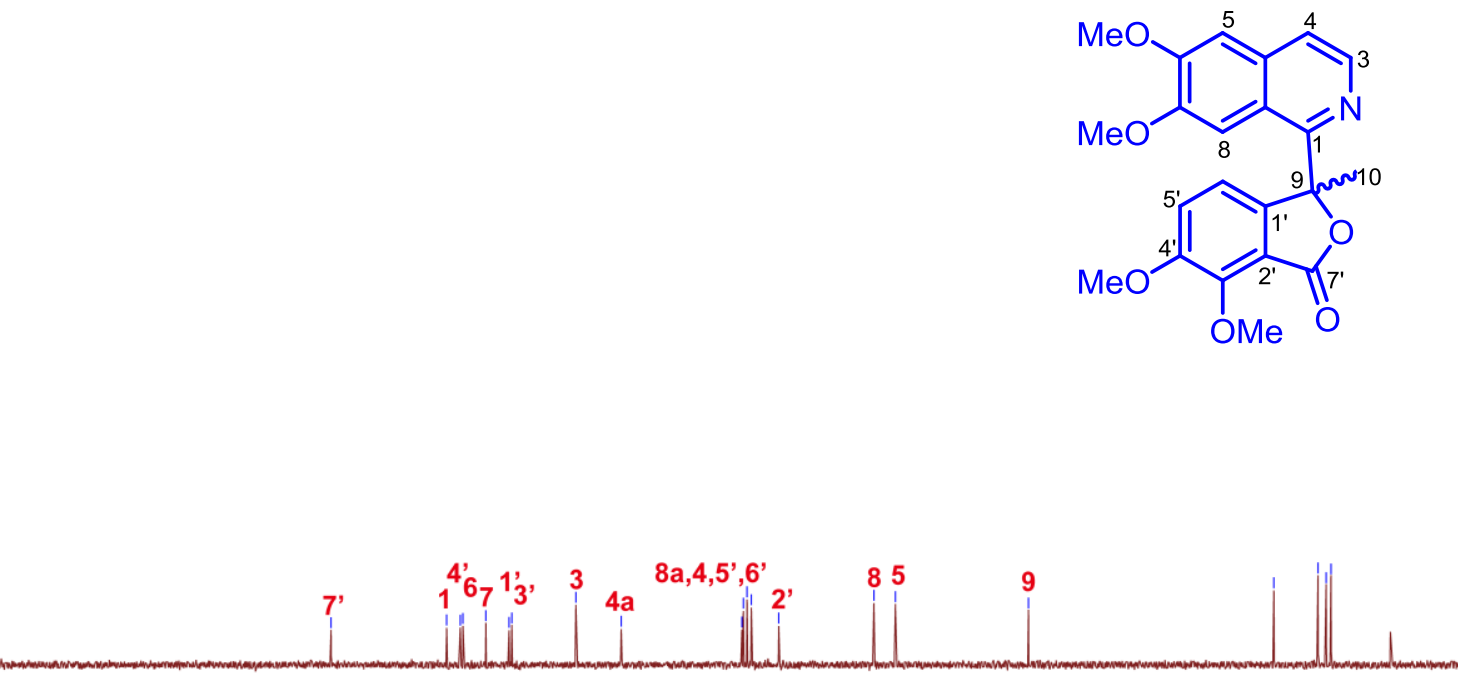

10

Figure S20. ${ }^{13} \mathrm{C}$ NMR spectrum of compound 2 in DMSO- $d_{6}(150 \mathrm{MHz})$ 


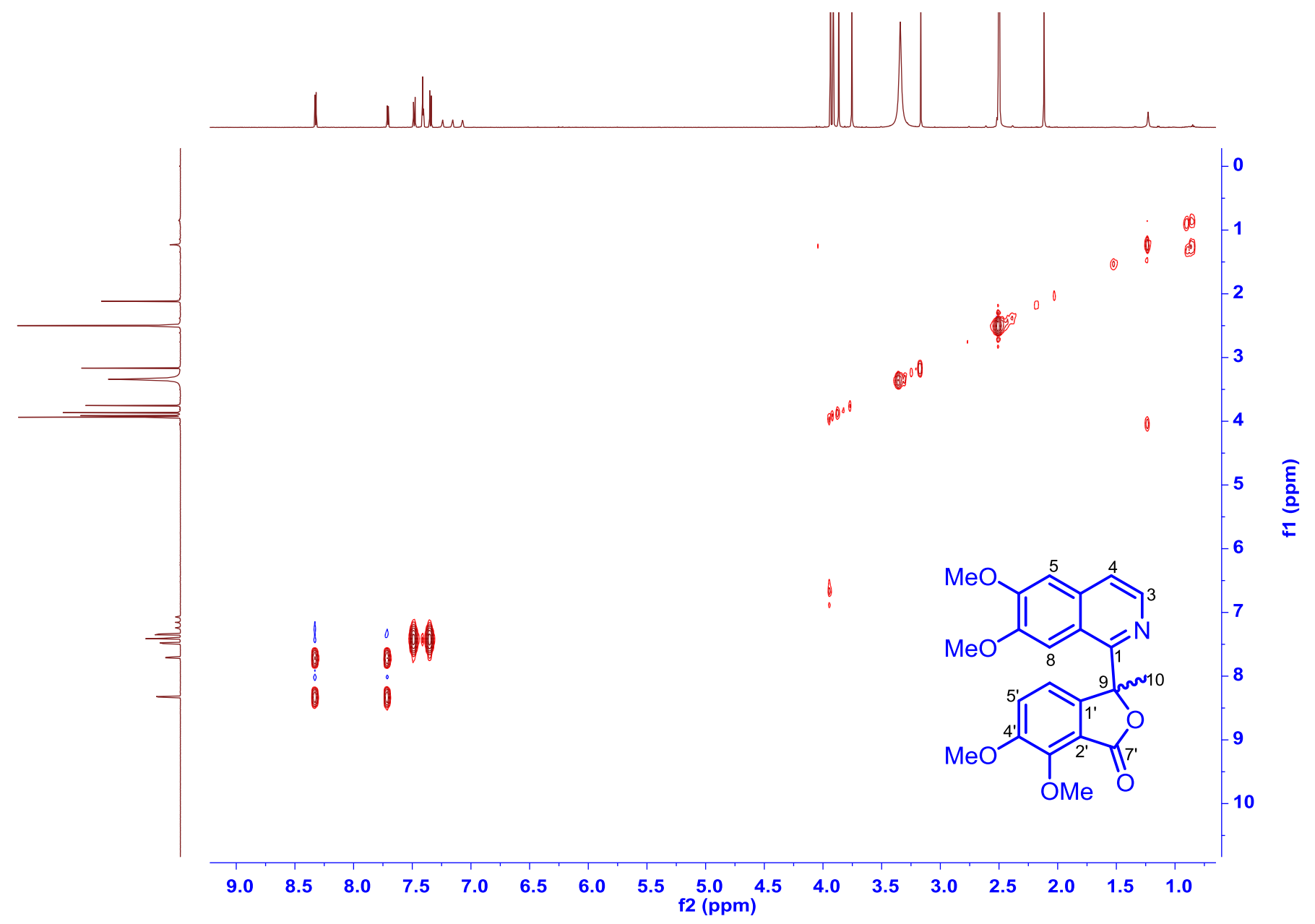

Figure S21. The ${ }^{1} \mathrm{H}-{ }^{1} \mathrm{H}$ COSY spectrum of compound 2 in DMSO-d $(600 \mathrm{MHz})$ 


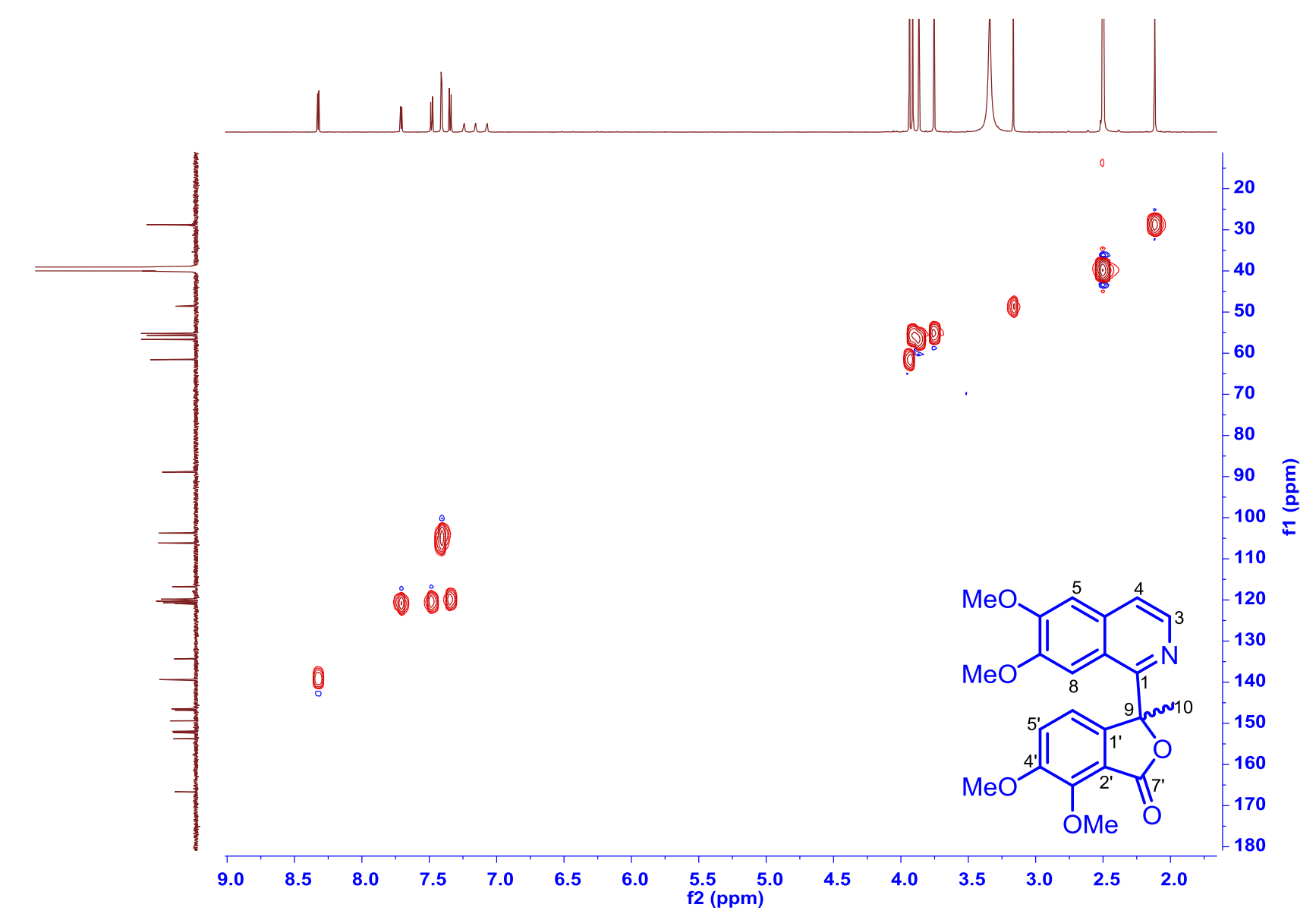

Figure S22. The HSQC spectrum of compound 2 in DMSO-d $(600 \mathrm{MHz})$ 


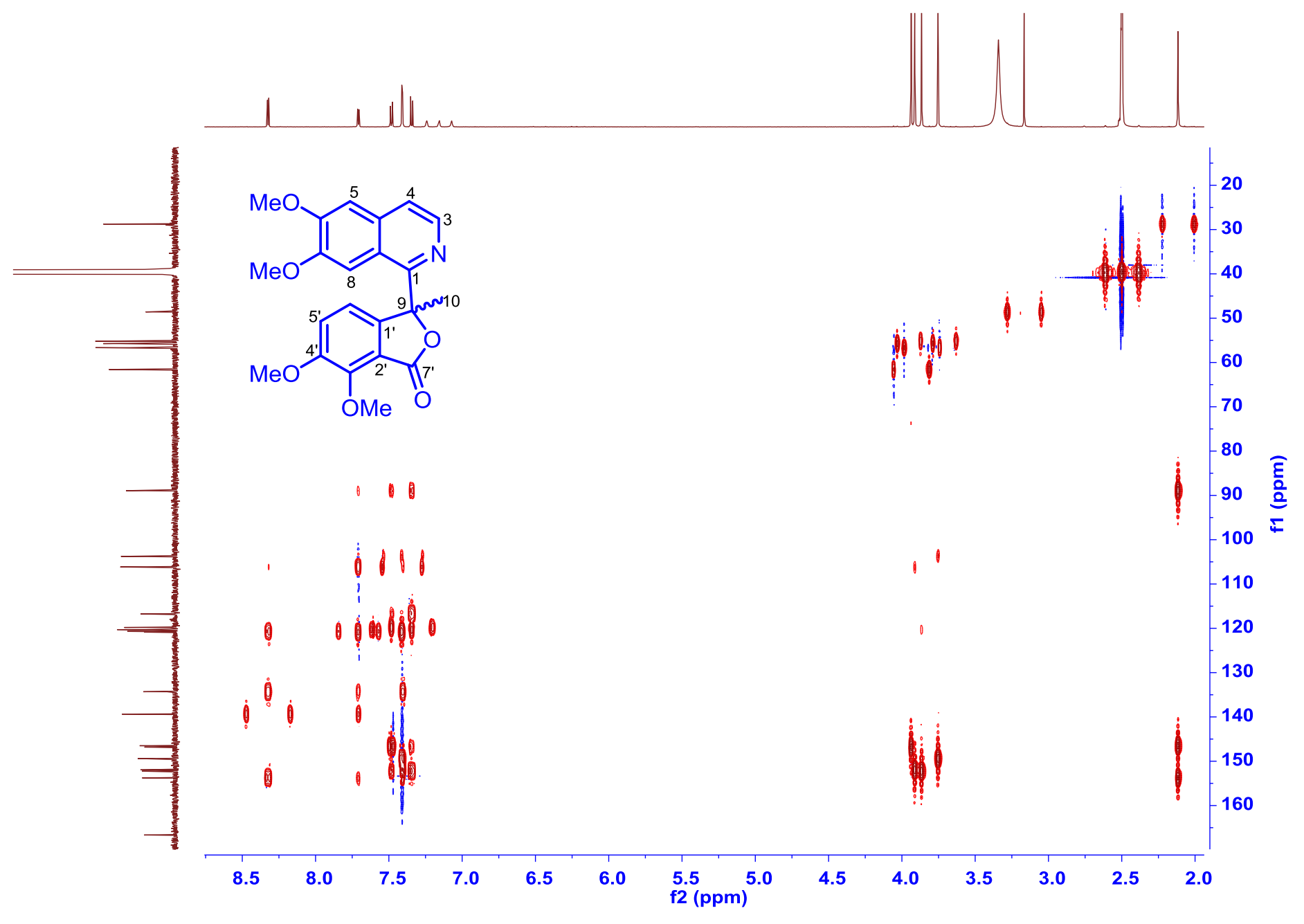

Figure S23. The HMBC spectrum of compound 2 in DMSO- $d_{6}(600 M H z)$ 


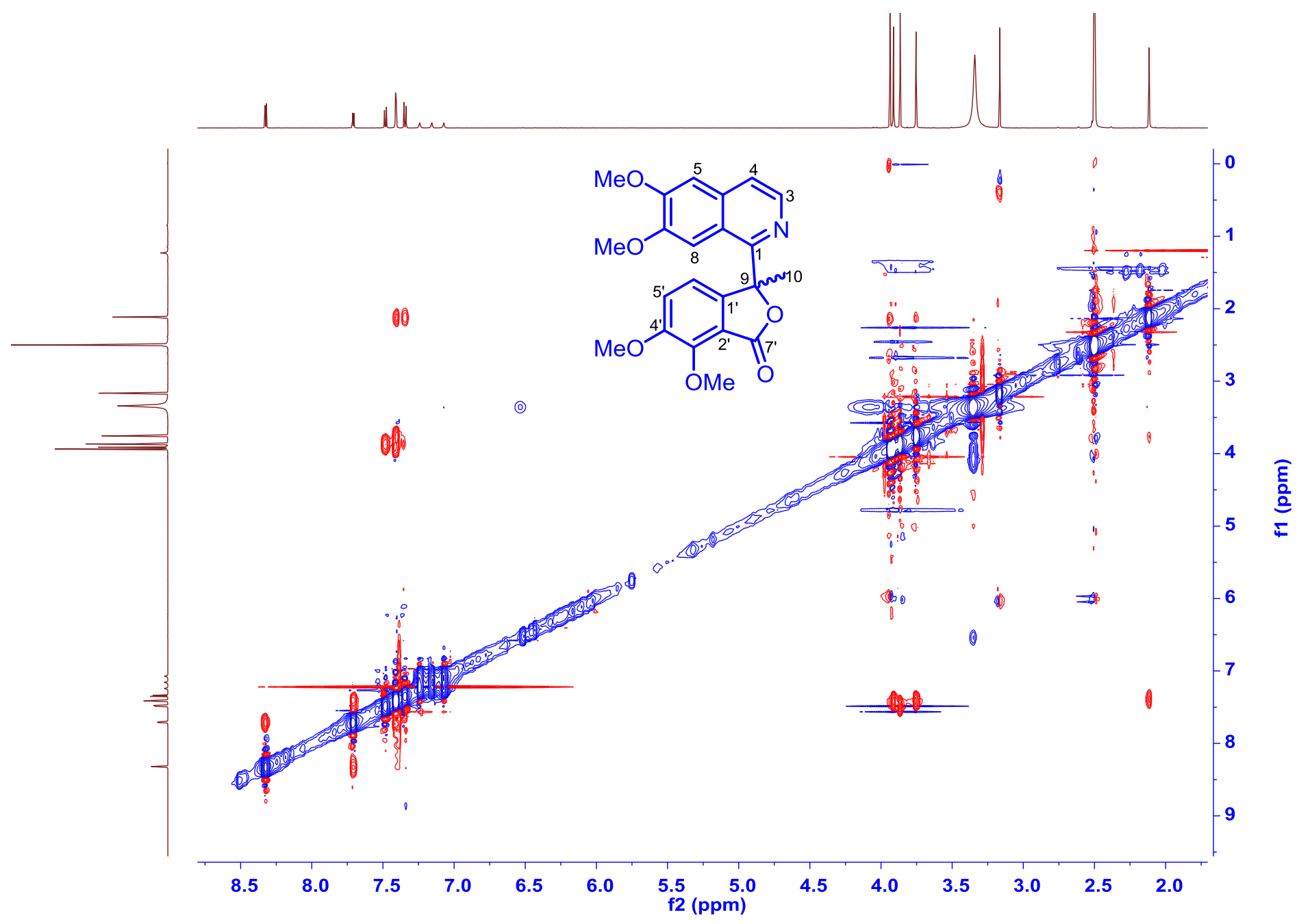

Figure S24. The NOESY spectrum of compound 2 in DMSO- $d_{6}(600 \mathrm{MHz})$ 


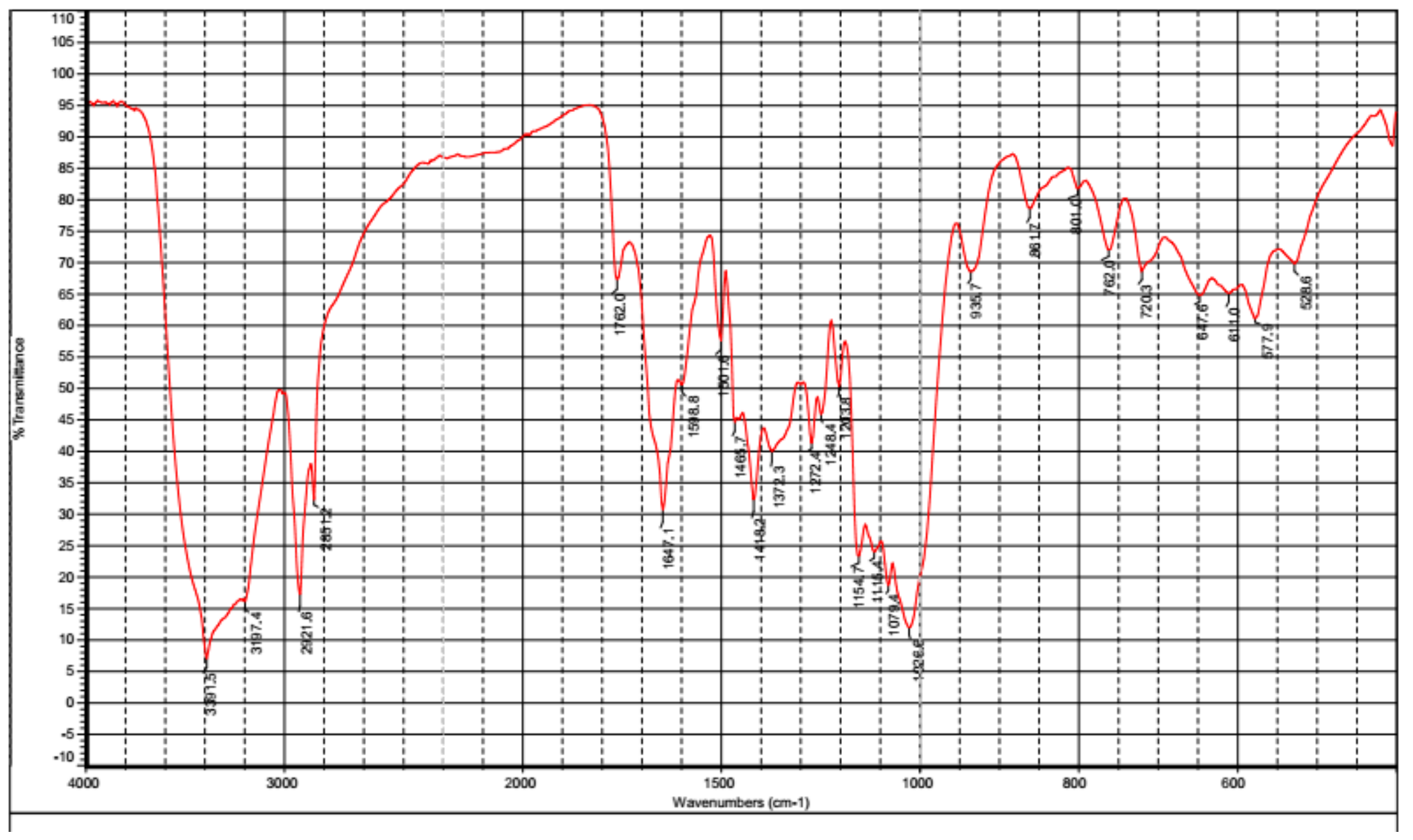

Figure S25. The IR spectrum of compound 2 


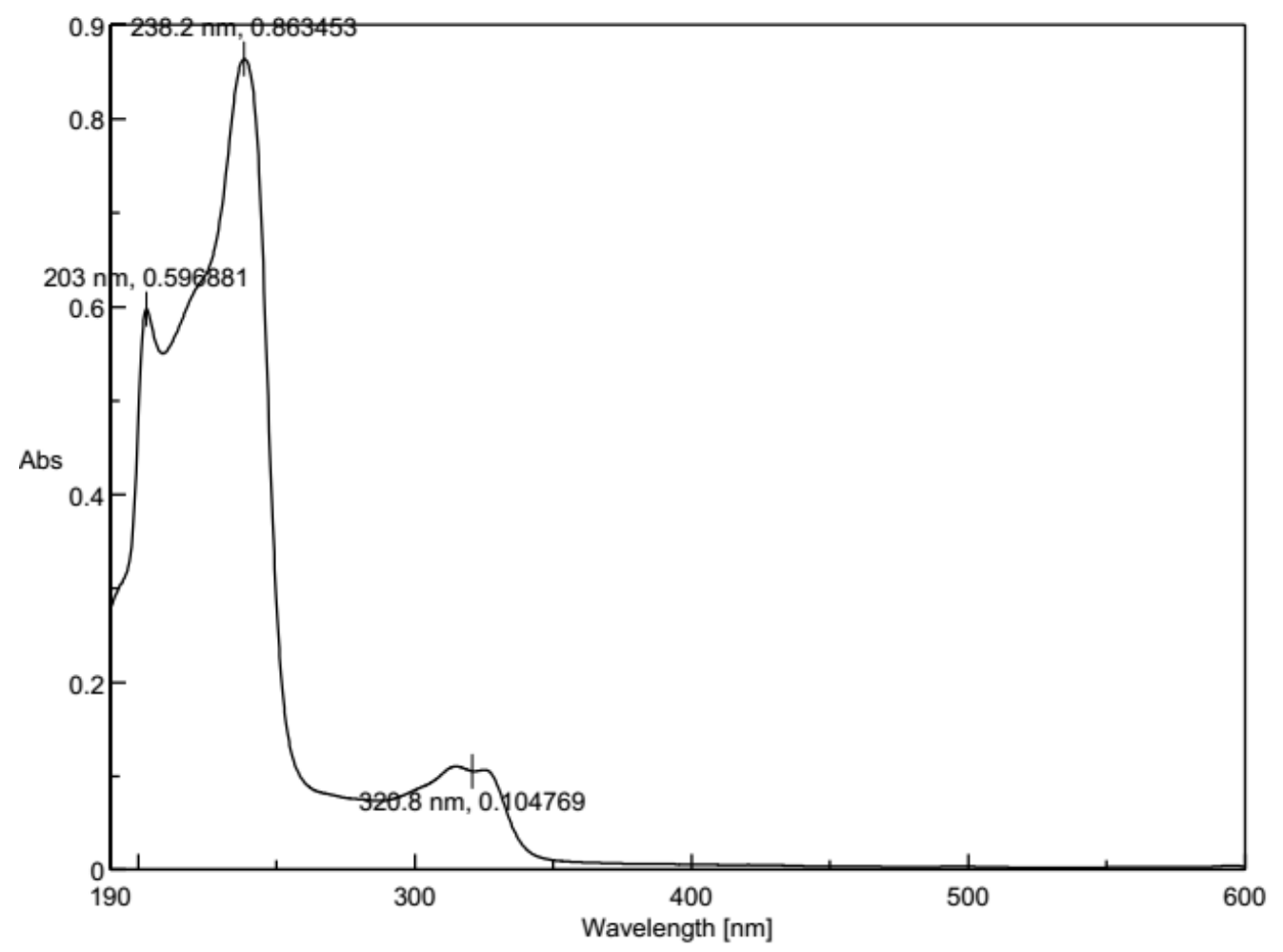

Figure S26. The UV spectrum of compound 2 in $\mathrm{MeOH}$

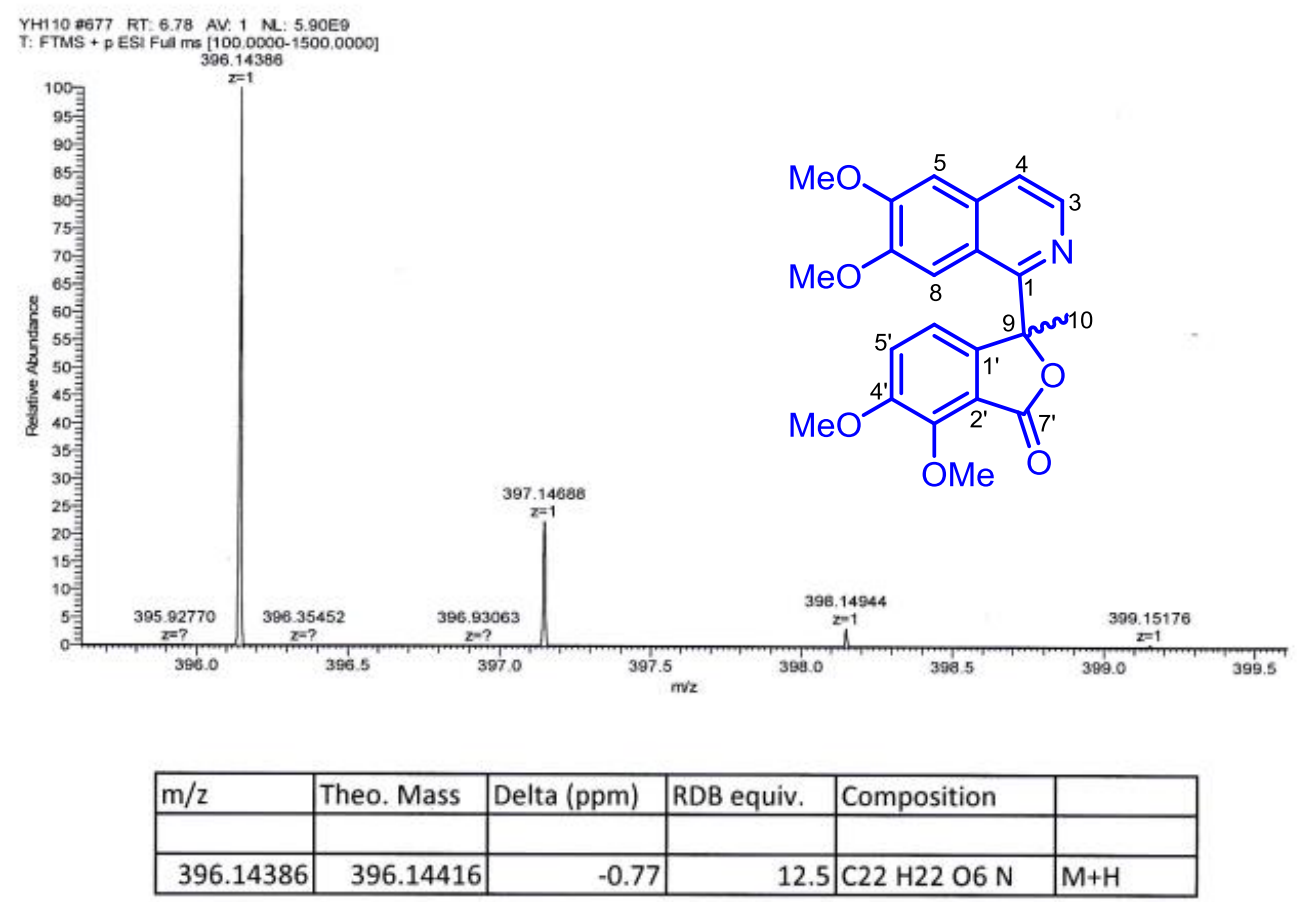

Figure S27. The HR-Mass spectrum of compound 2 in $\mathrm{MeOH}$ 


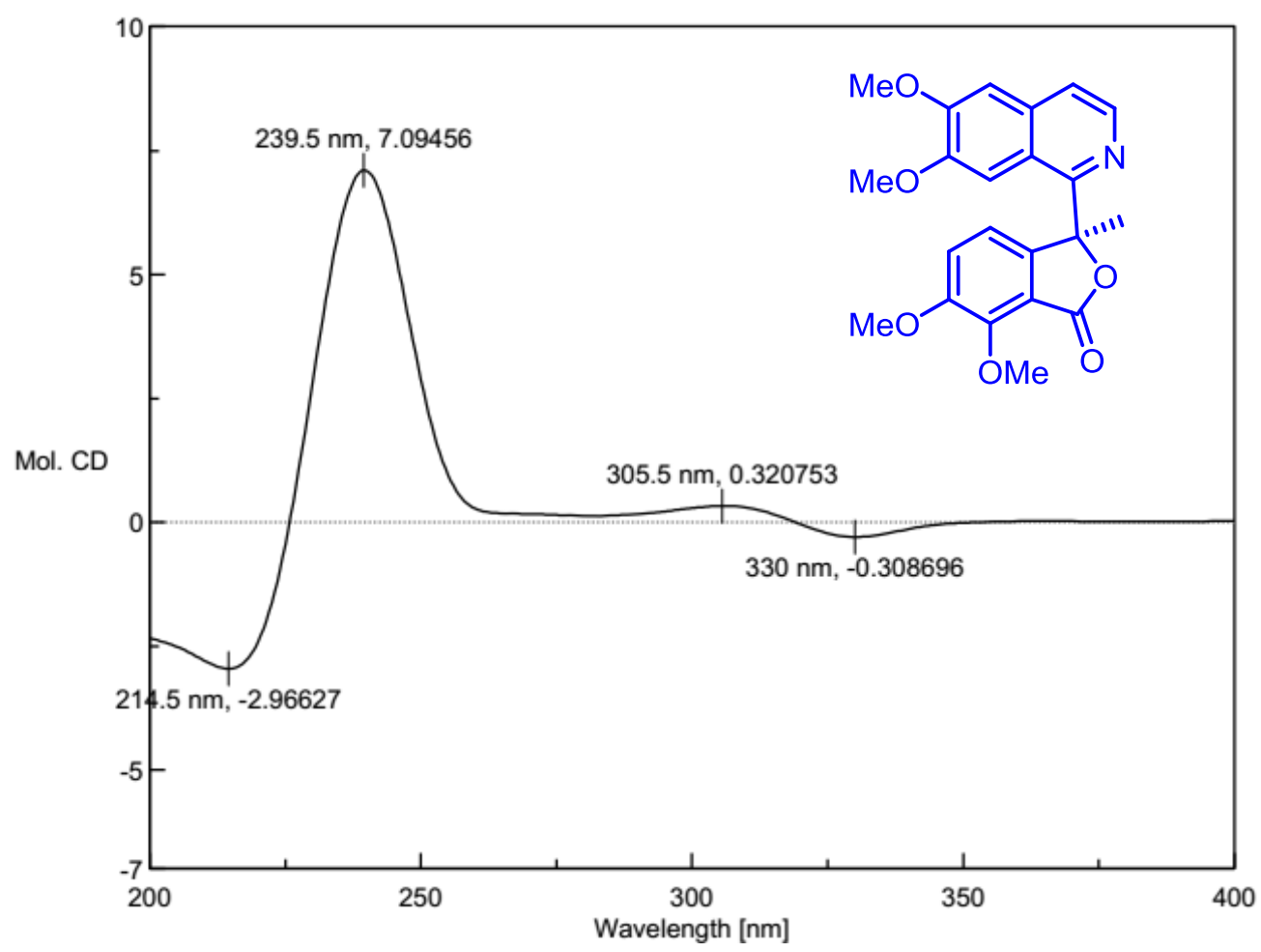

Figure S28. The CD spectrum of compound (+)-2 in $\mathrm{MeOH}$

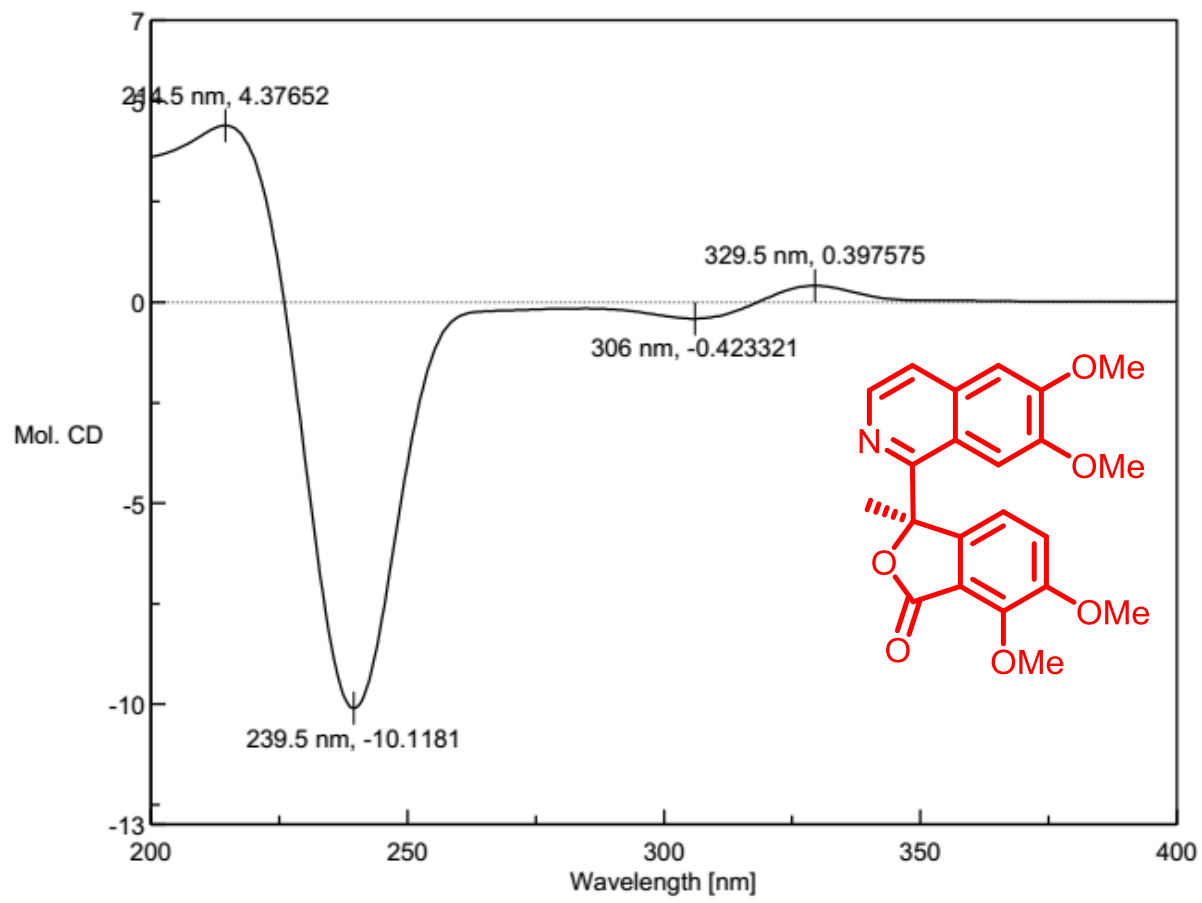

Figure S29. The CD spectrum of compound (-)-2 in $\mathrm{MeOH}$ 


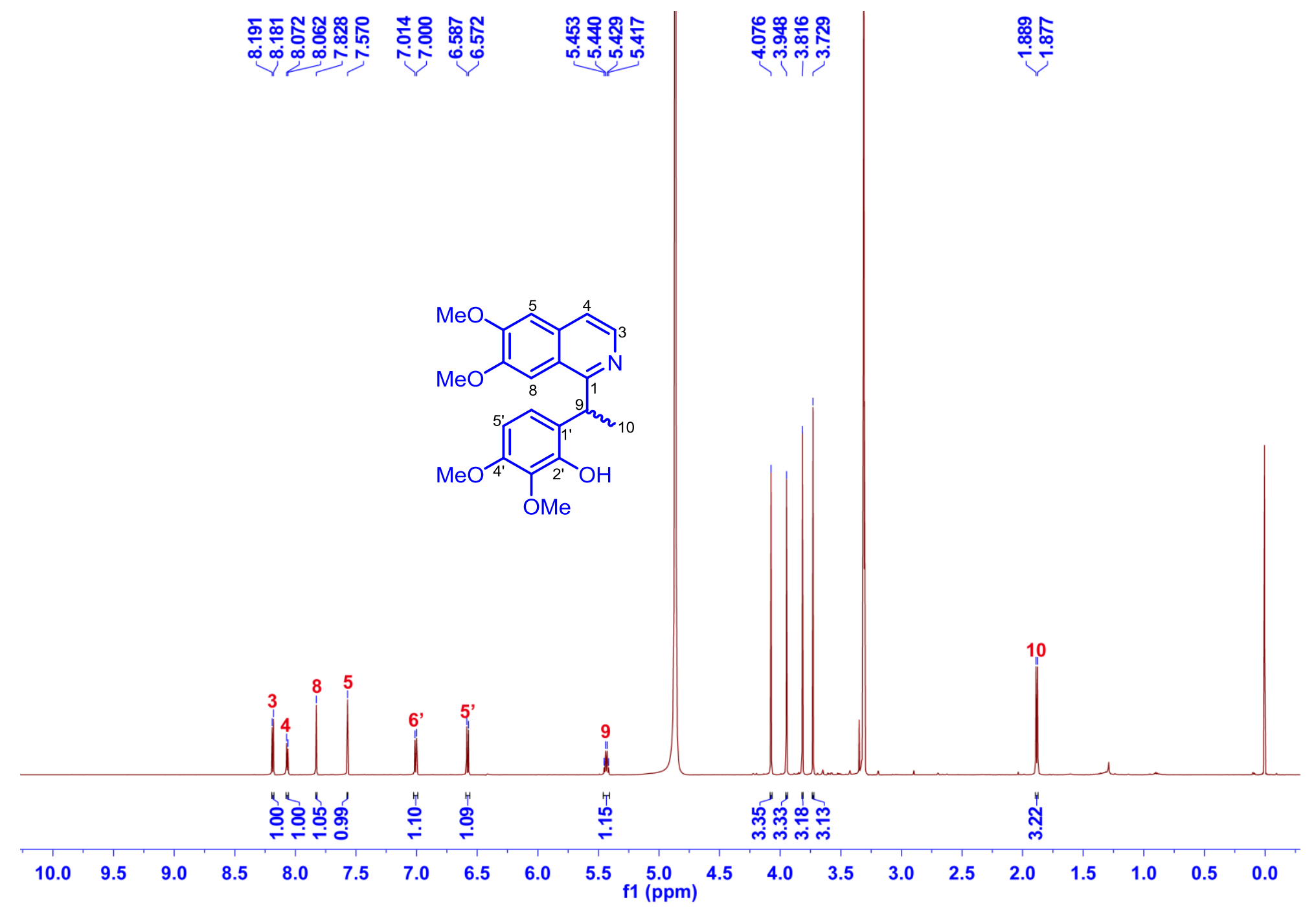

Figure S30. ${ }^{1} \mathrm{H}$ NMR spectrum of compound 3 in $\mathrm{MeOH}-d_{4}(600 \mathrm{MHz})$ 


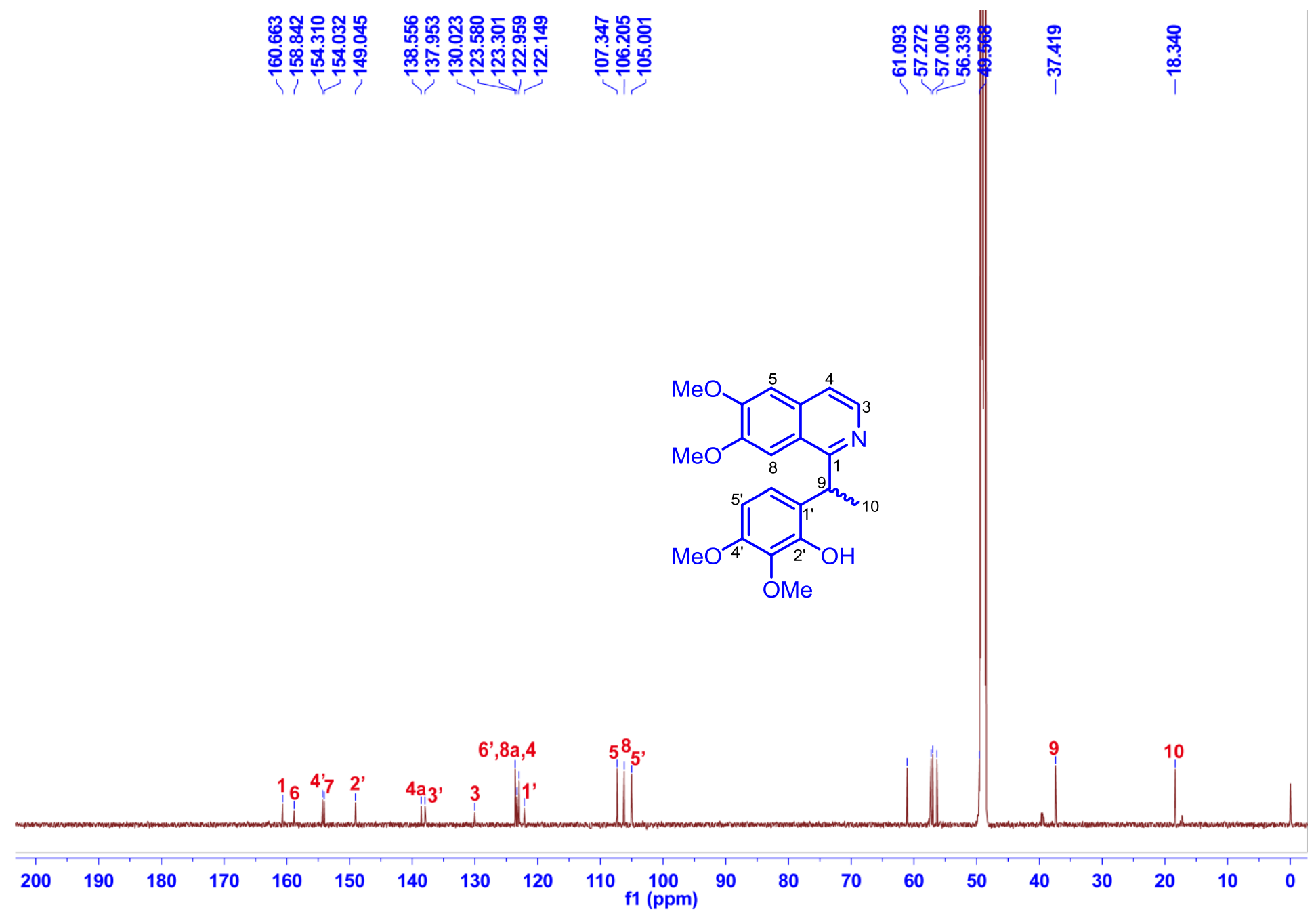

Figure S31. ${ }^{13} \mathrm{C}$ NMR spectrum of compound 3 in $\mathrm{MeOH}-d_{4}(150 \mathrm{MHz})$ 


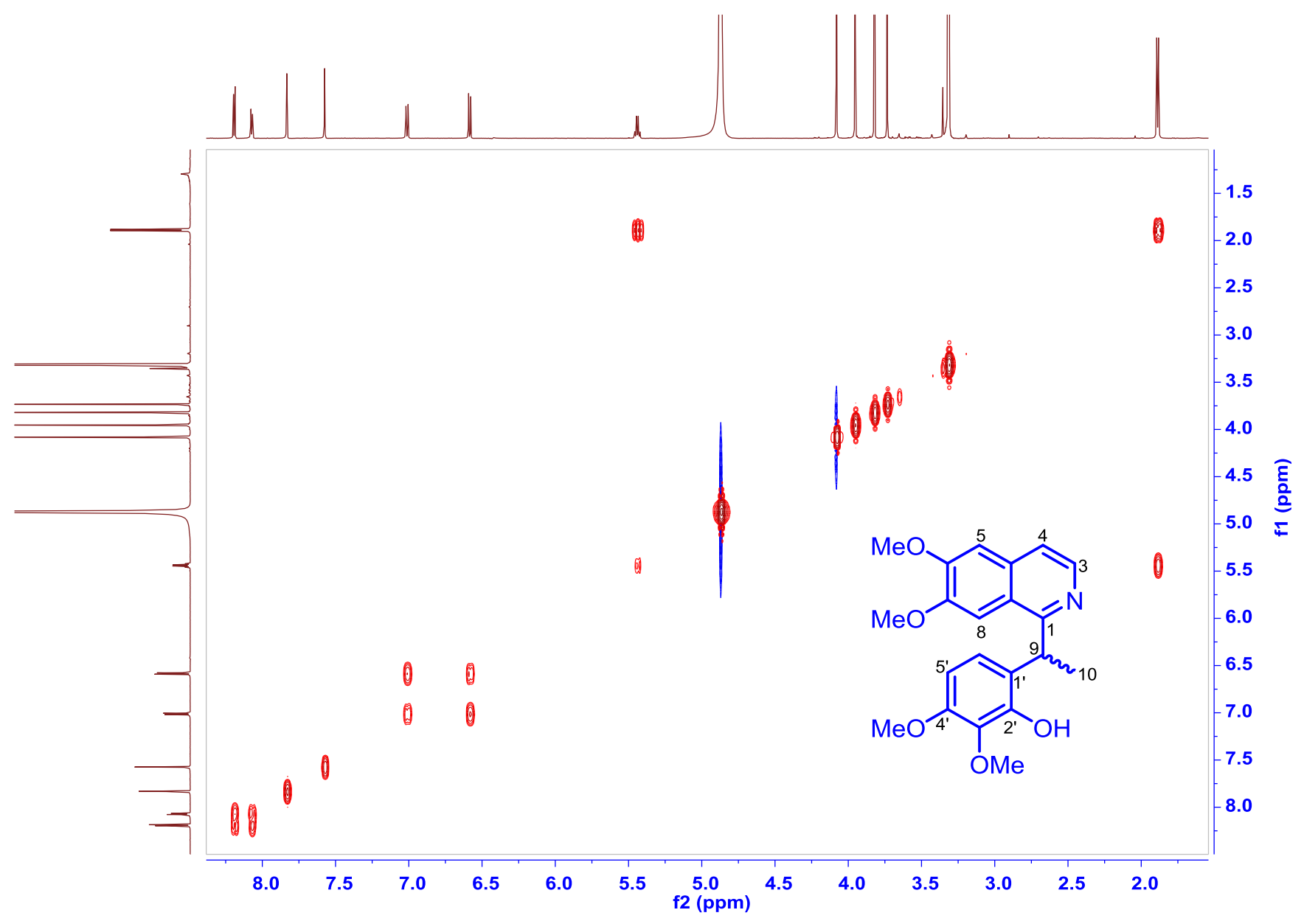

Figure S32. The ${ }^{1} \mathrm{H}-{ }^{1} \mathrm{H}$ COSY spectrum of compound 3 in $\mathrm{MeOH}-d_{4}(600 \mathrm{MHz})$ 


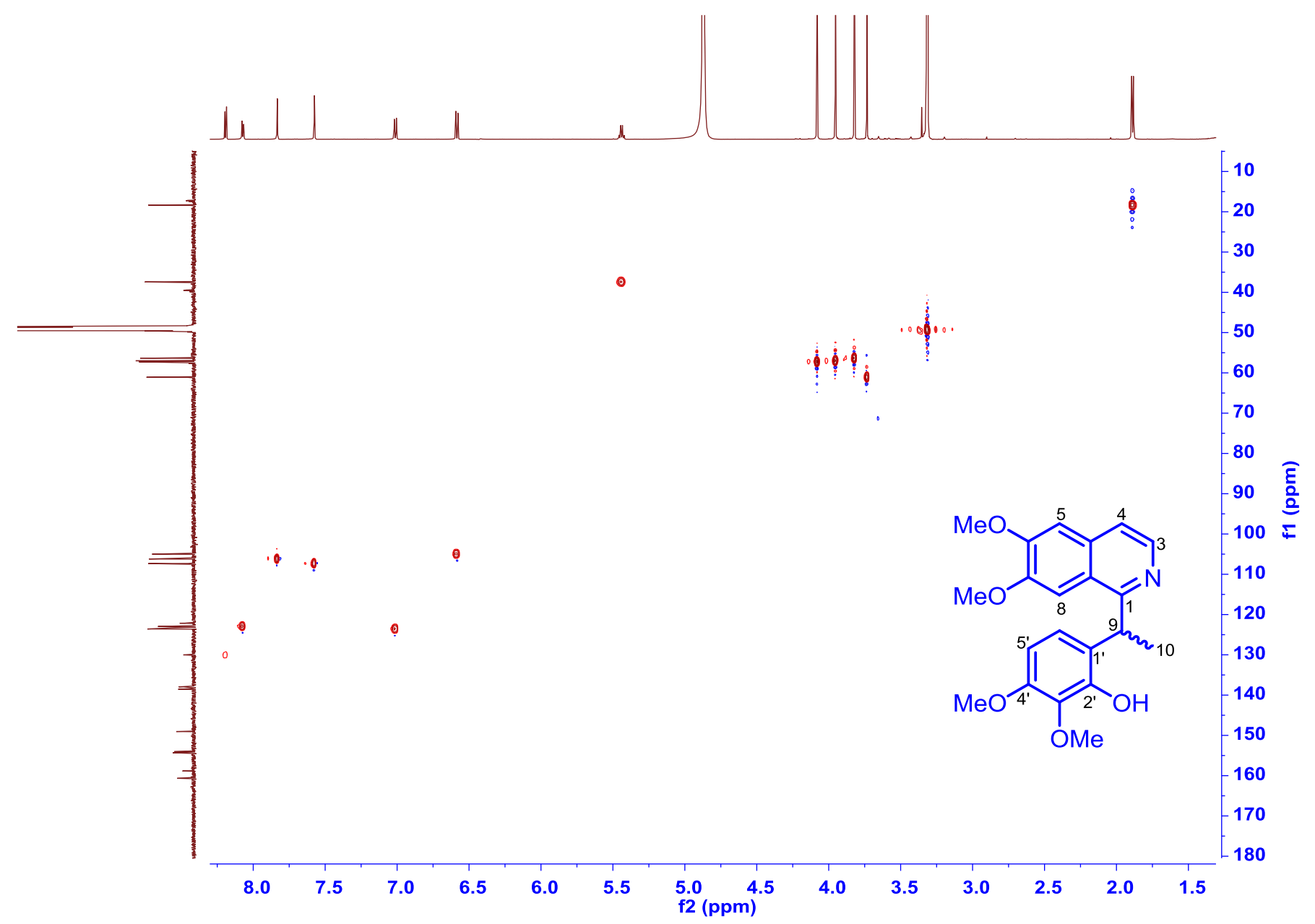

Figure S33. The HSQC spectrum of compound 3 in $\mathrm{MeOH}-d_{4}(600 \mathrm{MHz})$ 


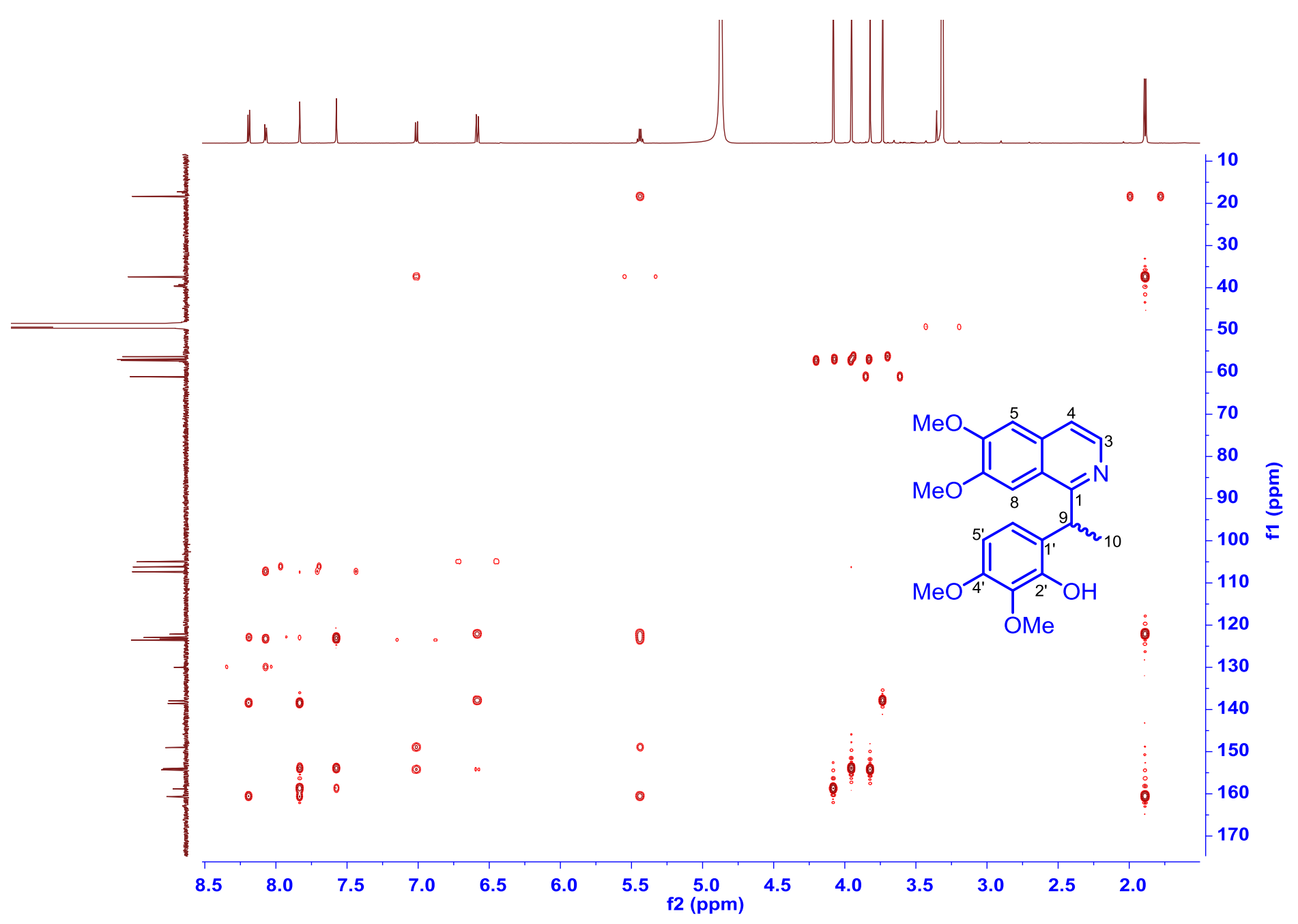

Figure S34. The HMBC spectrum of compound 3 in MeOH- $d_{4}(600 M H z)$ 


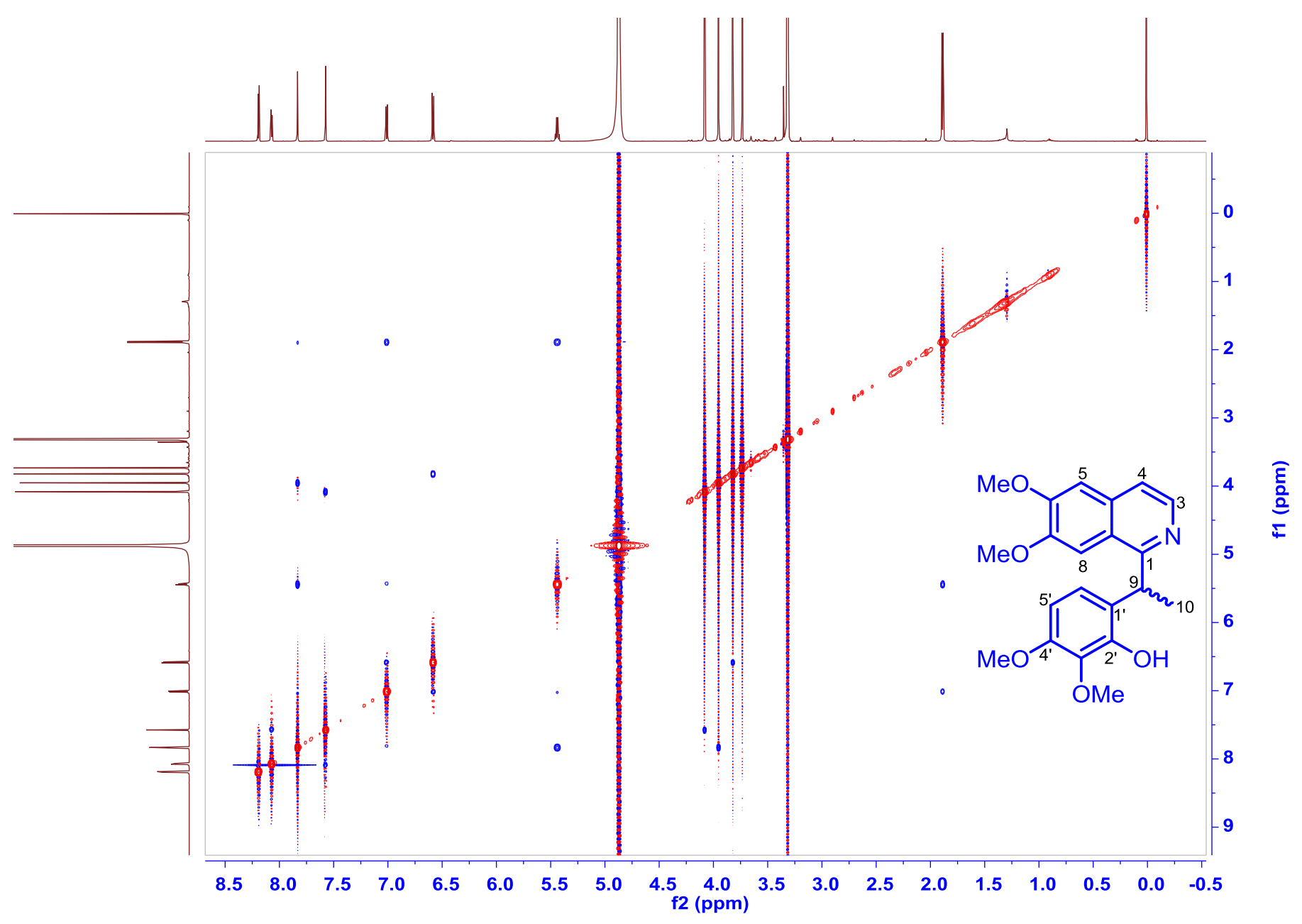

Figure S35. The NOESY spectrum of compound 3 in MeOH-d $(600 \mathrm{MHz})$ 


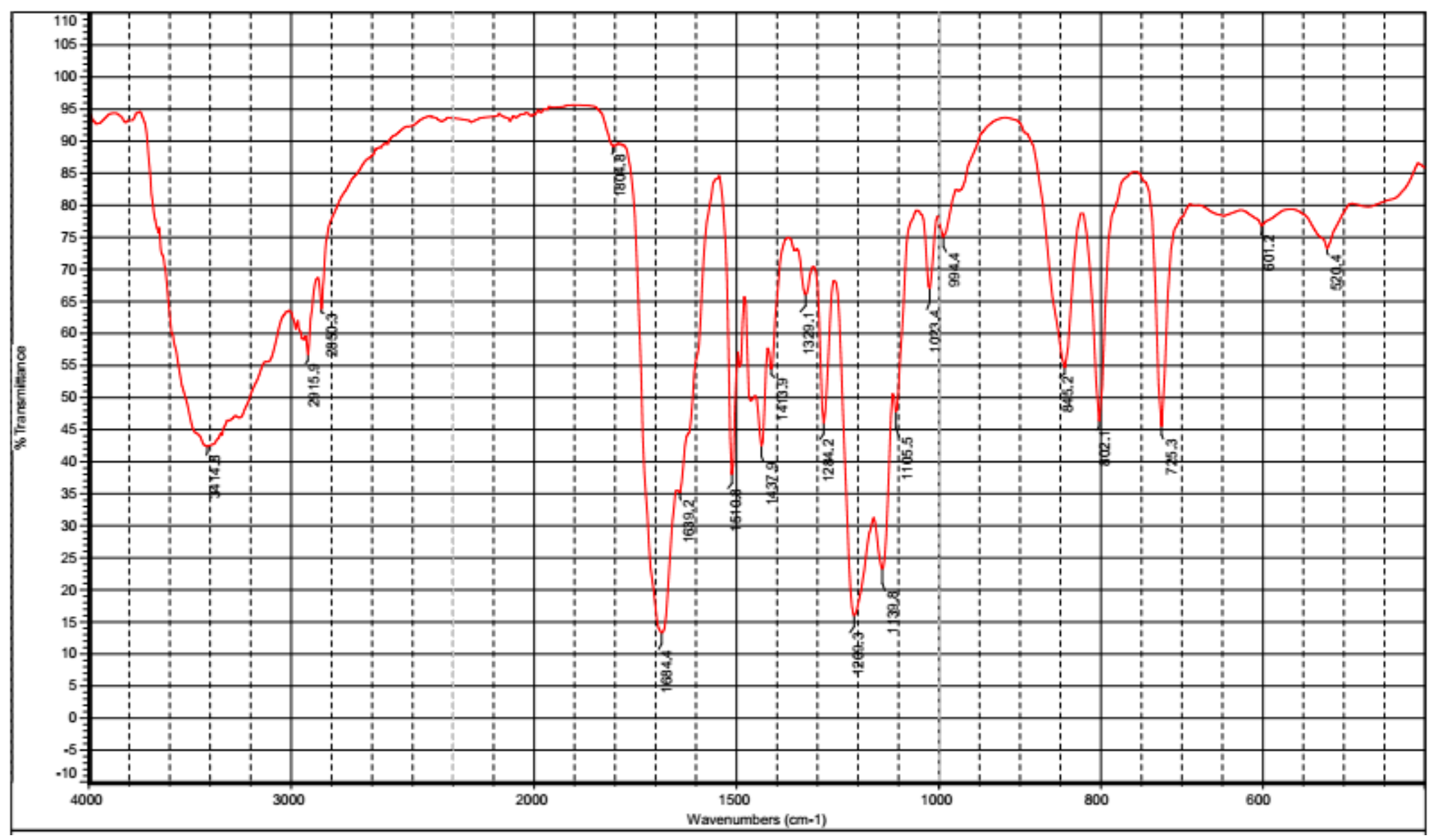

Figure S36. The IR spectrum of compound 3 


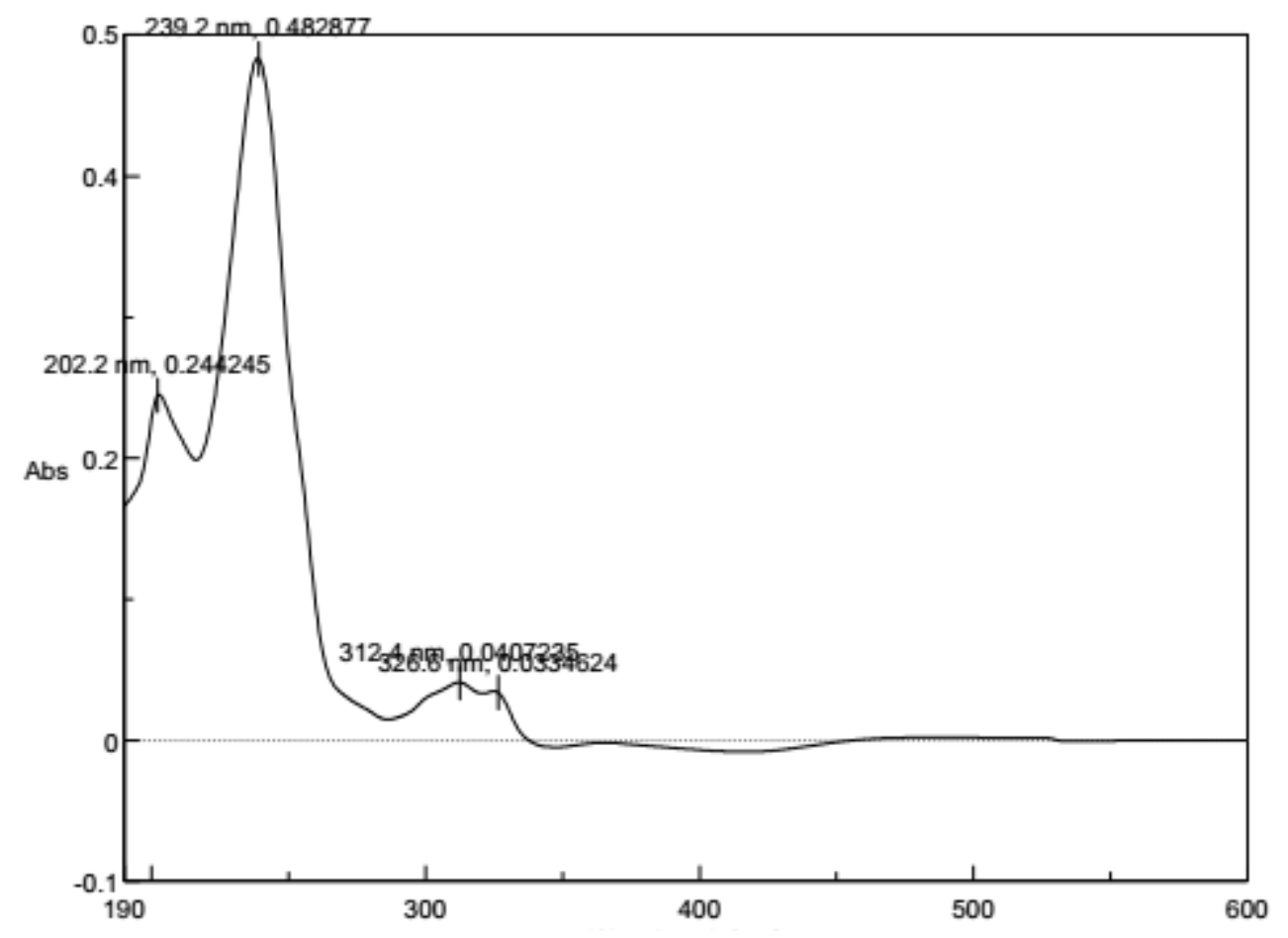

Figure S37. The UV spectrum of compound 3 in $\mathrm{MeOH}$

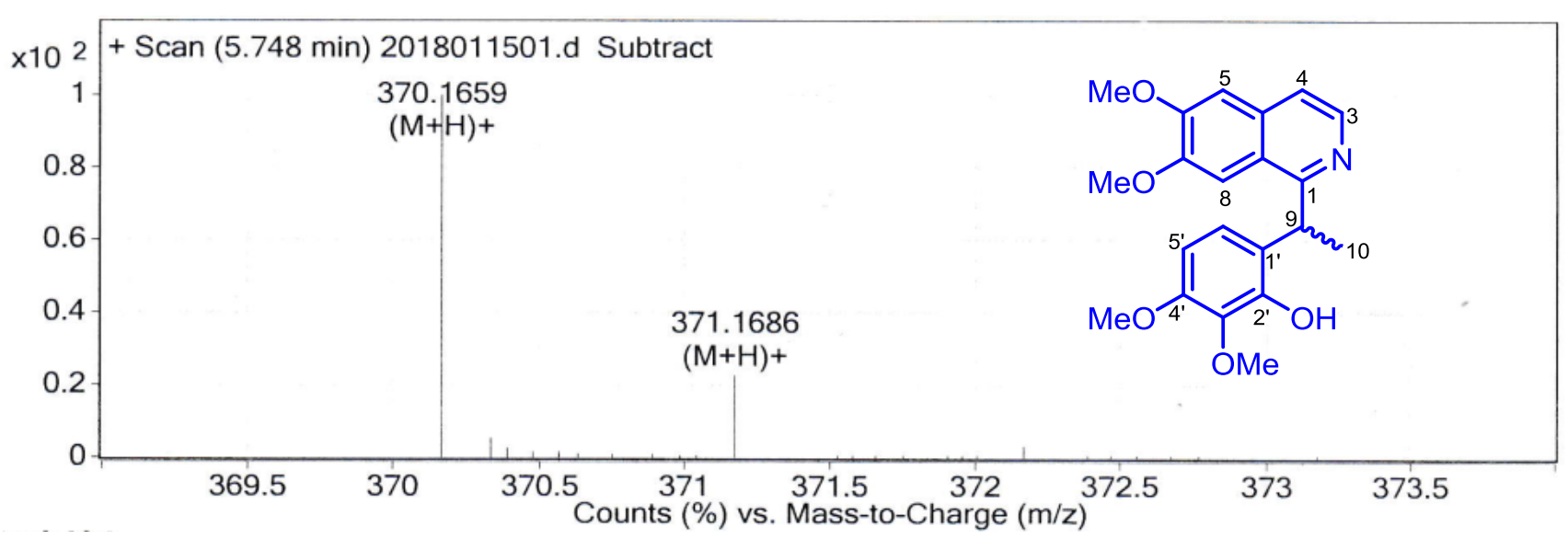

MS Formula Results: + Scan (5.748 min) Sub (2018011501.d)

\begin{tabular}{|c|c|c|c|c|c|c|c|c|c|c|c|c|c|c|}
\hline \multicolumn{2}{|r|}{$m / 2$} & Ion & Formula & Abundance & & & & & & & & & & \\
\hline & 370.1659 & $(M+H)+$ & $\mathrm{C} 21 \mathrm{H} 24 \mathrm{~N} \mathrm{O5}$ & 592738.2 & & & & & & & & & & \\
\hline & Best & Formula (M) & Ion Formula & Score & Cross Sco & Mass & Calc Mass & Catc $m / z$ & Diff (ppm) & Abs Diff (ppm) & Mass Match & Abund Match & Spacing Match & DBE \\
\hline - & $\checkmark$ & $\mathrm{C} 21 \mathrm{H} 23 \mathrm{NOS}$ & $\mathrm{C} 21 \mathrm{H} 24 \mathrm{~N} \mathrm{O5}$ & 99.84 & & 369.1587 & 369.1576 & 370.1649 & $\begin{array}{r}-2.8 \\
\end{array}$ & 28 & 99.75 & 99.94 & 99.92 & 11 \\
\hline - & $\Gamma$ & C18H27N O5S & C18 H28 N O5S & 98.32 & & 369.1587 & 369.161 & 370.1683 & 6.33 & 6.33 & 98.71 & 96.65 & 99.54 & 6 \\
\hline - & $\Gamma$ & $\mathrm{C} 13 \mathrm{H} 27 \mathrm{~N} 307 \mathrm{~S}$ & $\mathrm{C} 13 \mathrm{H} 28 \mathrm{~N} 307 \mathrm{~S}$ & 97.32 & & 369.1587 & 369.157 & 370.1642 & -4.58 & 4.58 & 99.32 & 92.18 & $\begin{array}{l}99.5 \\
\end{array}$ & 2 \\
\hline - & $\Gamma$ & $\mathrm{C} 22 \mathrm{H} 27 \mathrm{~N} \mathrm{~S} 2$ & $\mathrm{C} 22 \mathrm{H} 28 \mathrm{~N} \mathrm{~S} 2$ & 97.28 & & 369.1587 & 369.1585 & 370.1658 & -0.45 & 0.45 & 99.99 & 91 & 99.38 & 10 \\
\hline +. & $\Gamma$ & $\mathrm{C} 25 \mathrm{H} 23 \mathrm{NS}$ & $\mathrm{C} 25 \mathrm{H} 24 \mathrm{NS}$ & 97.25 & & 369.1587 & 369.1551 & 370.1624 & -9.58 & 9.58 & $97.07 \mid$ & 95.55 & 99.64 & 15 \\
\hline
\end{tabular}

Figure S38. The HR-Mass spectrum of compound 3 in $\mathrm{MeOH}$ 


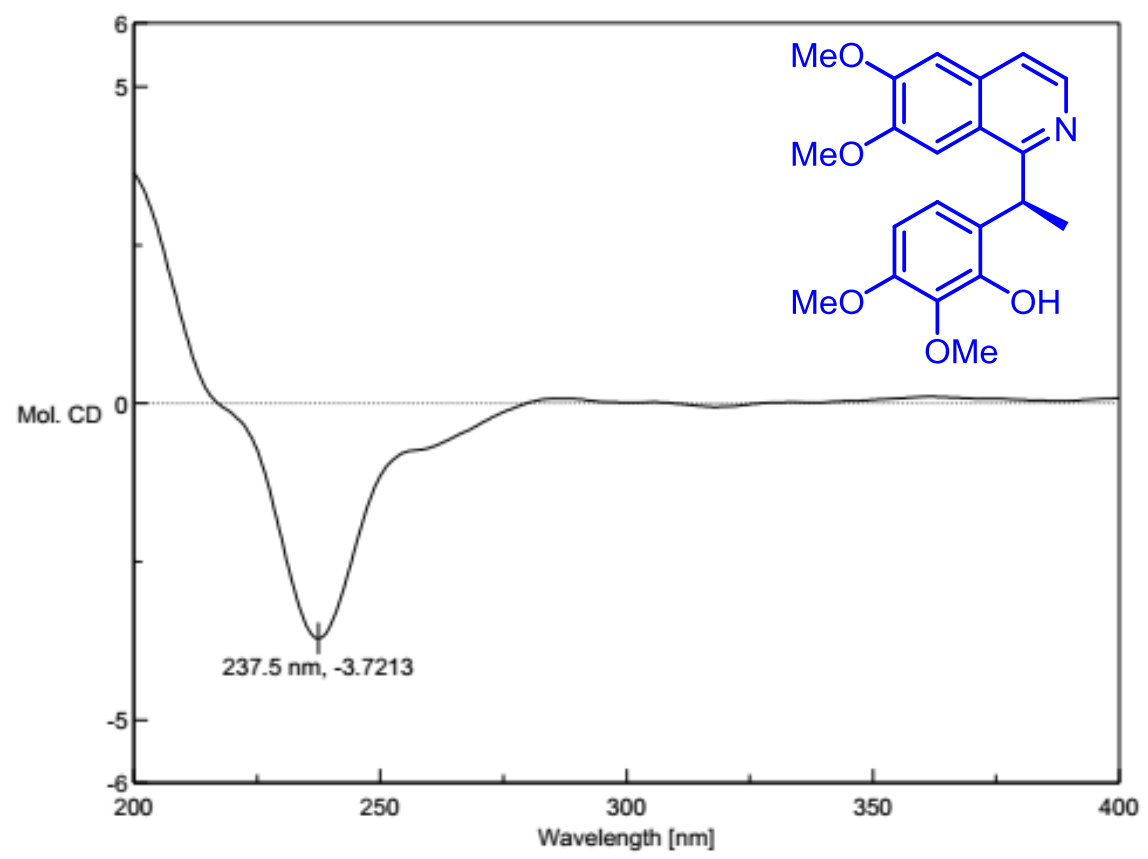

Figure S39. The CD spectrum of compound (+)-3 in $\mathrm{MeOH}$

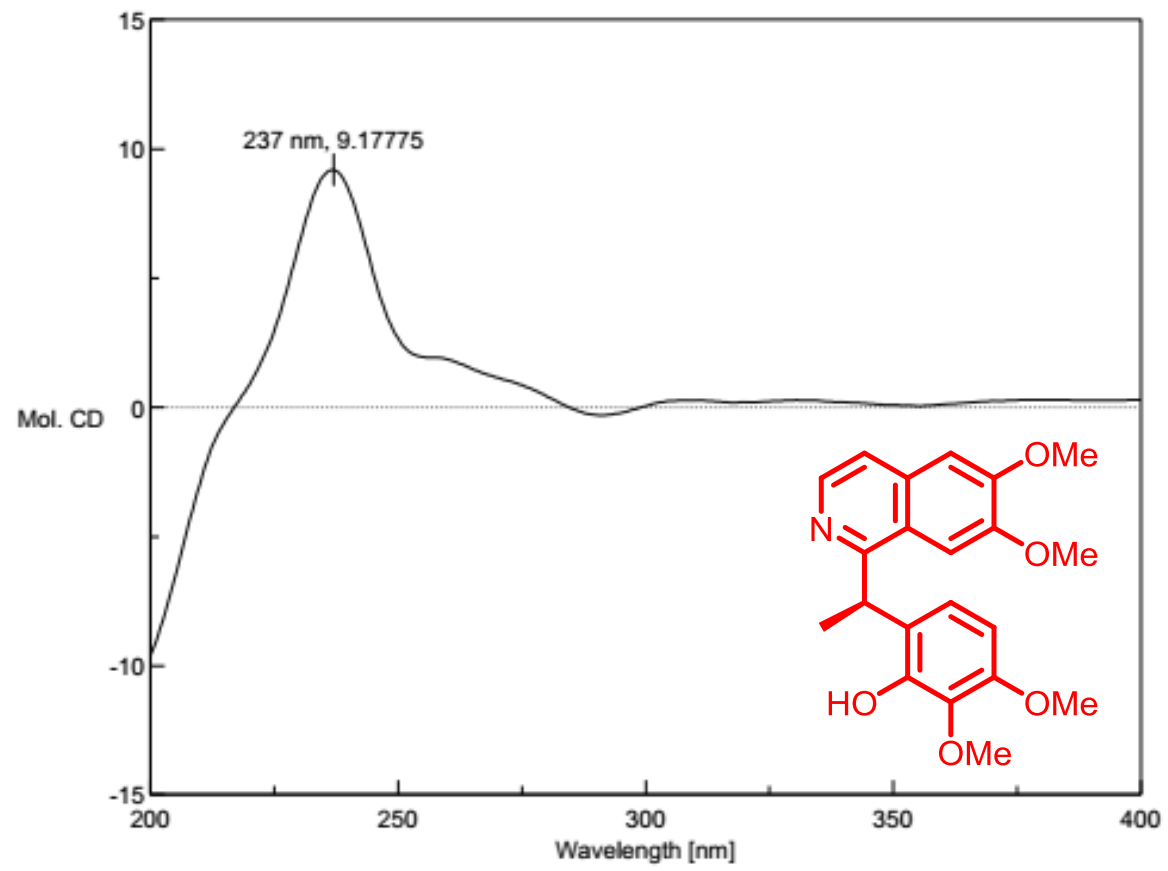

Figure S40. The CD spectrum of compound (-)-3 in $\mathrm{MeOH}$ 


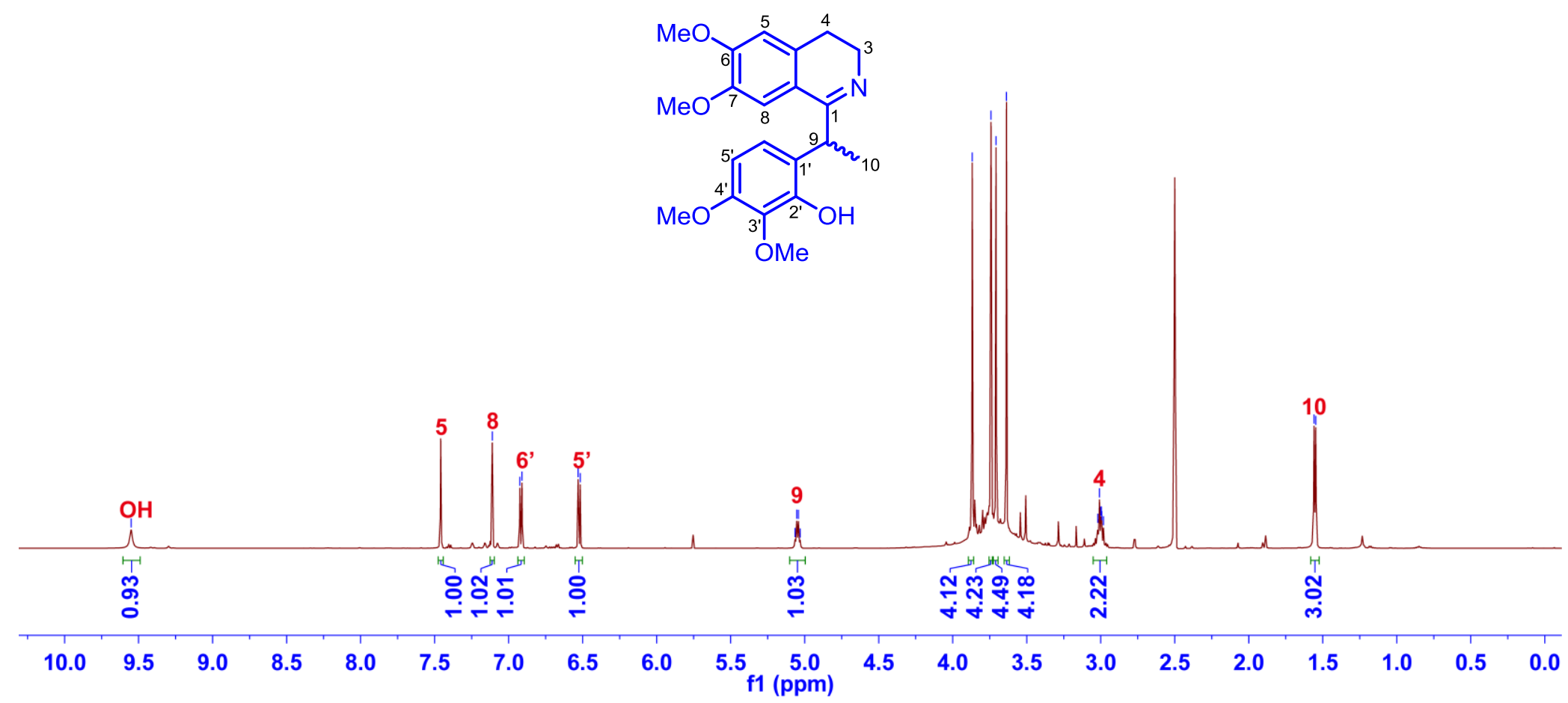

Figure S41. ${ }^{1} \mathrm{H}$ NMR spectrum of compound 4 in DMSO- $d_{6}(600 \mathrm{MHz})$ 


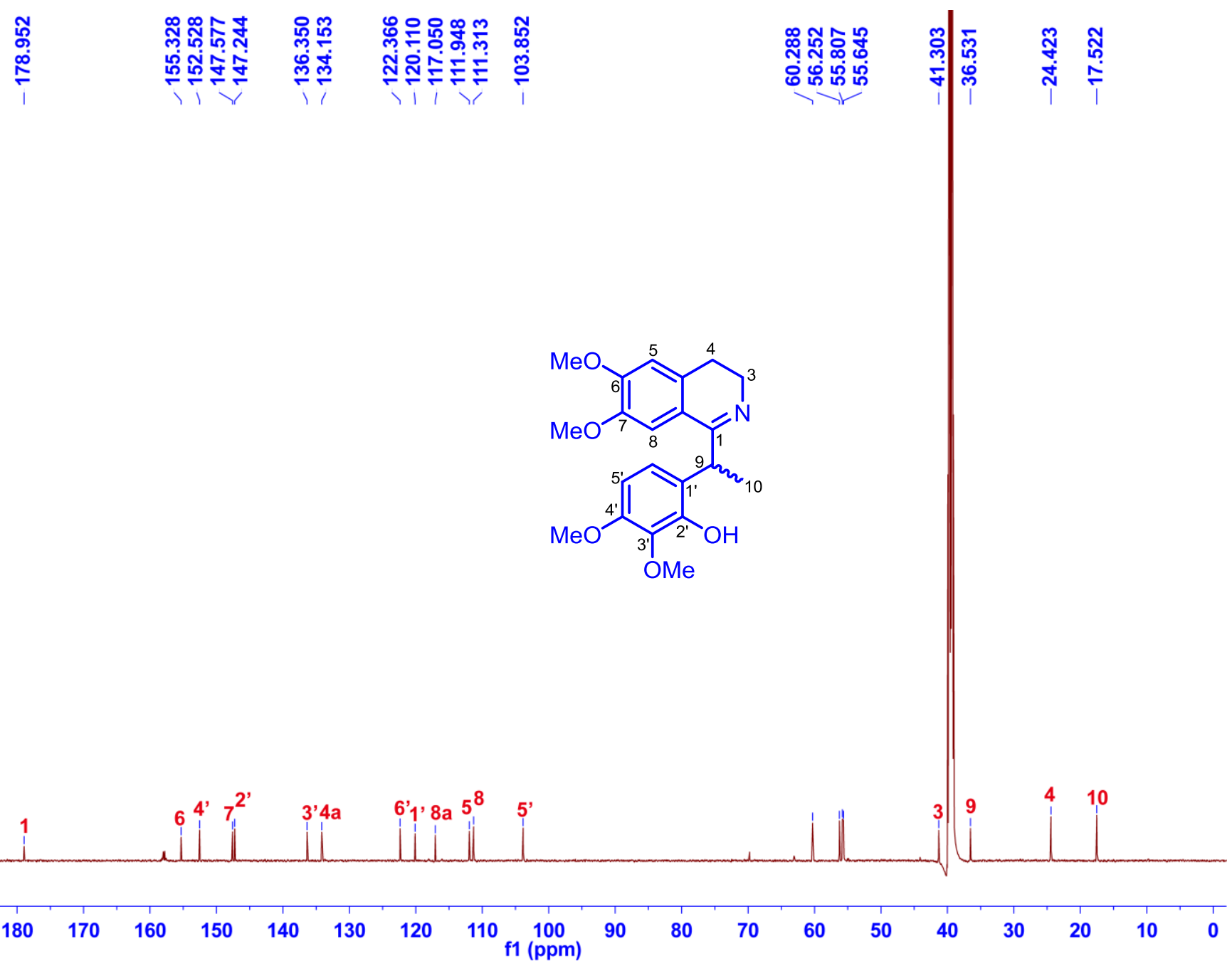

Figure S42. ${ }^{13} \mathrm{C}$ NMR spectrum of compound 4 in DMSO-d $(150 \mathrm{MHz})$ 


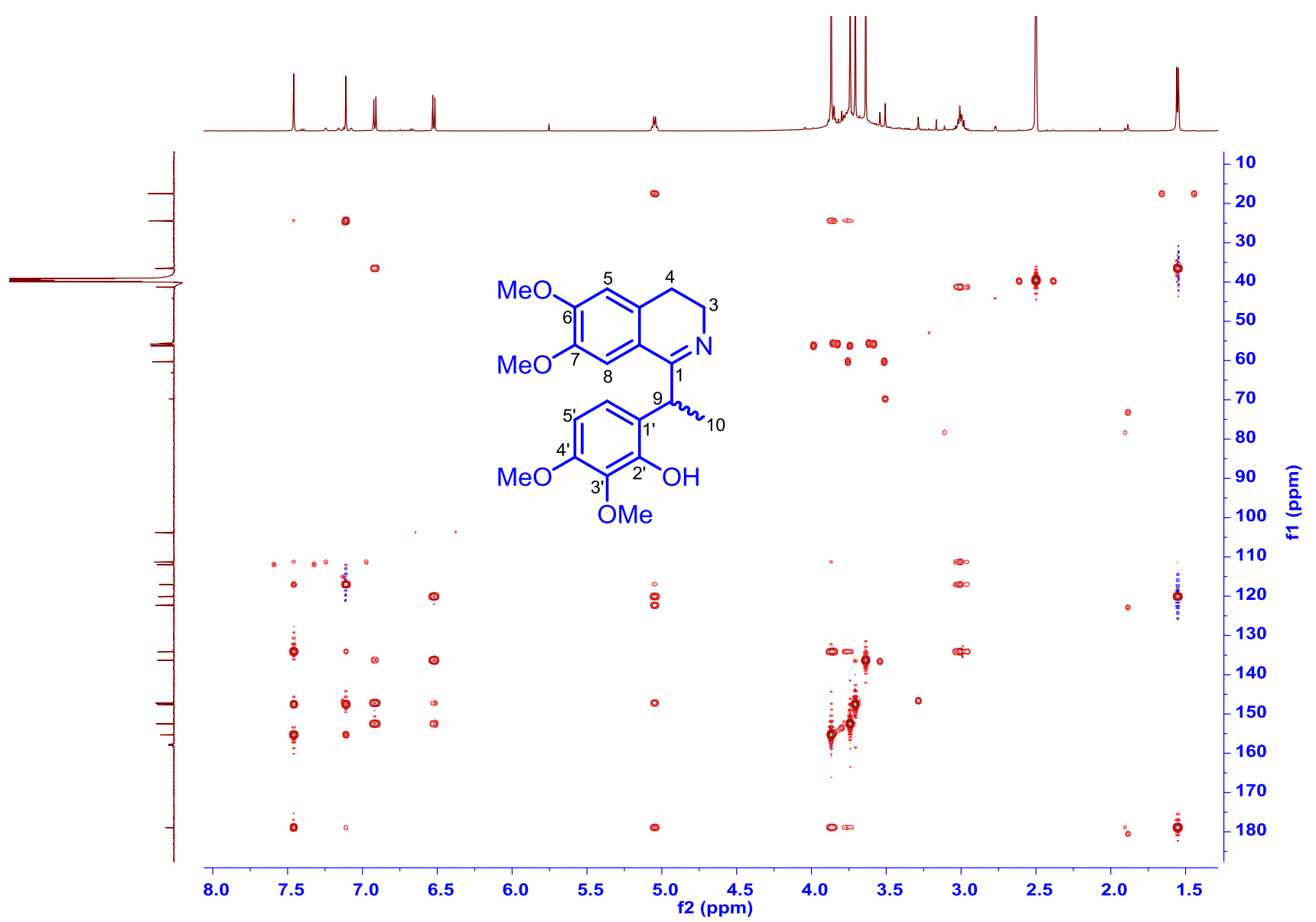

Figure S43. The HMBC spectrum of compound 4 in DMSO-d $(600 \mathrm{MHz})$ 


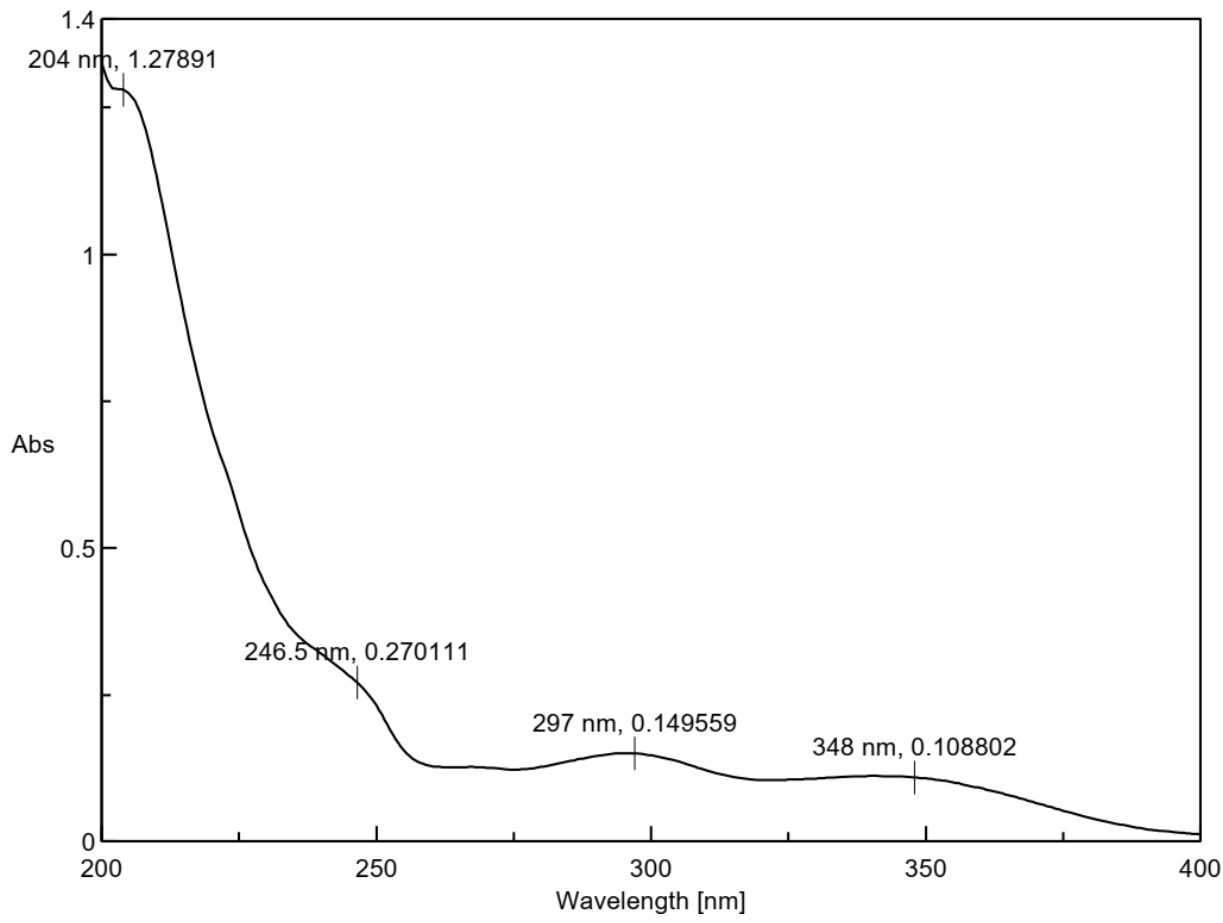

Figure S44.The UV spectrum of compound 4 in $\mathrm{MeOH}$
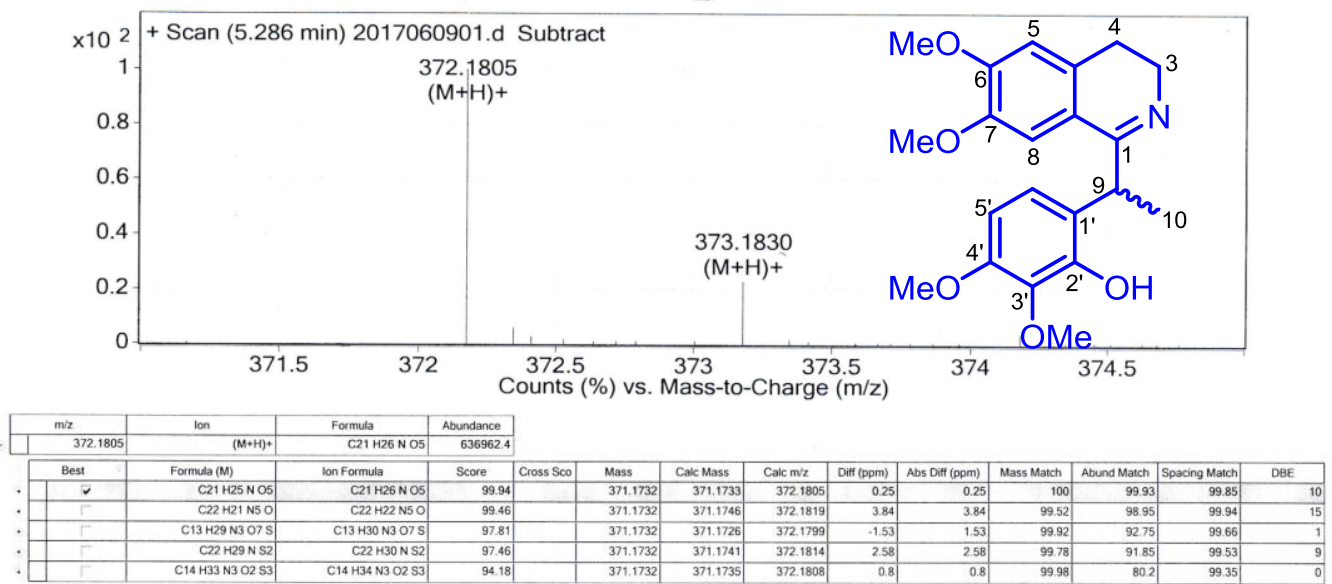

Figure S45.The HR-Mass spectrum of compound 4 in $\mathrm{MeOH}$ 


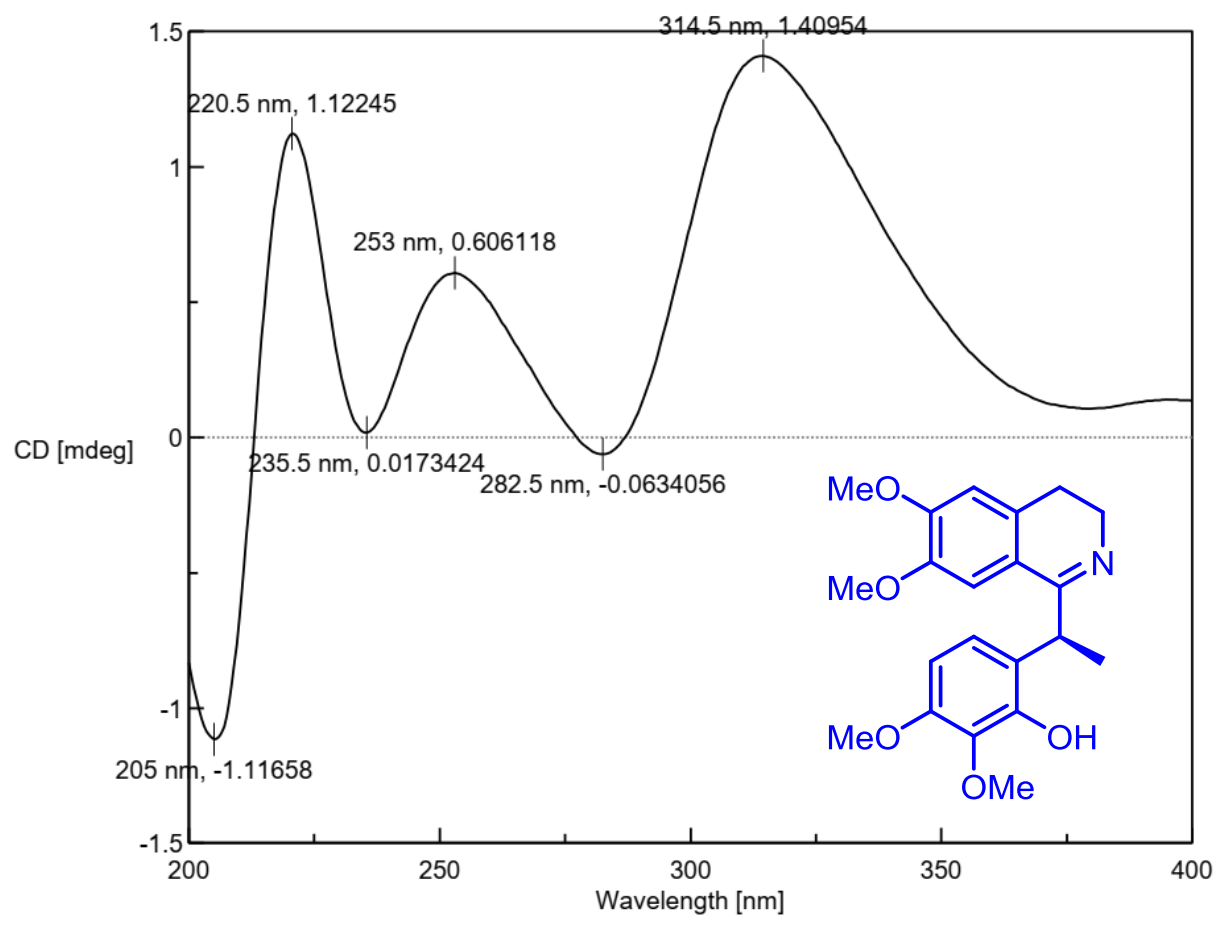

Figure S46. The CD spectrum of compound (+)-4 in $\mathrm{MeOH}$

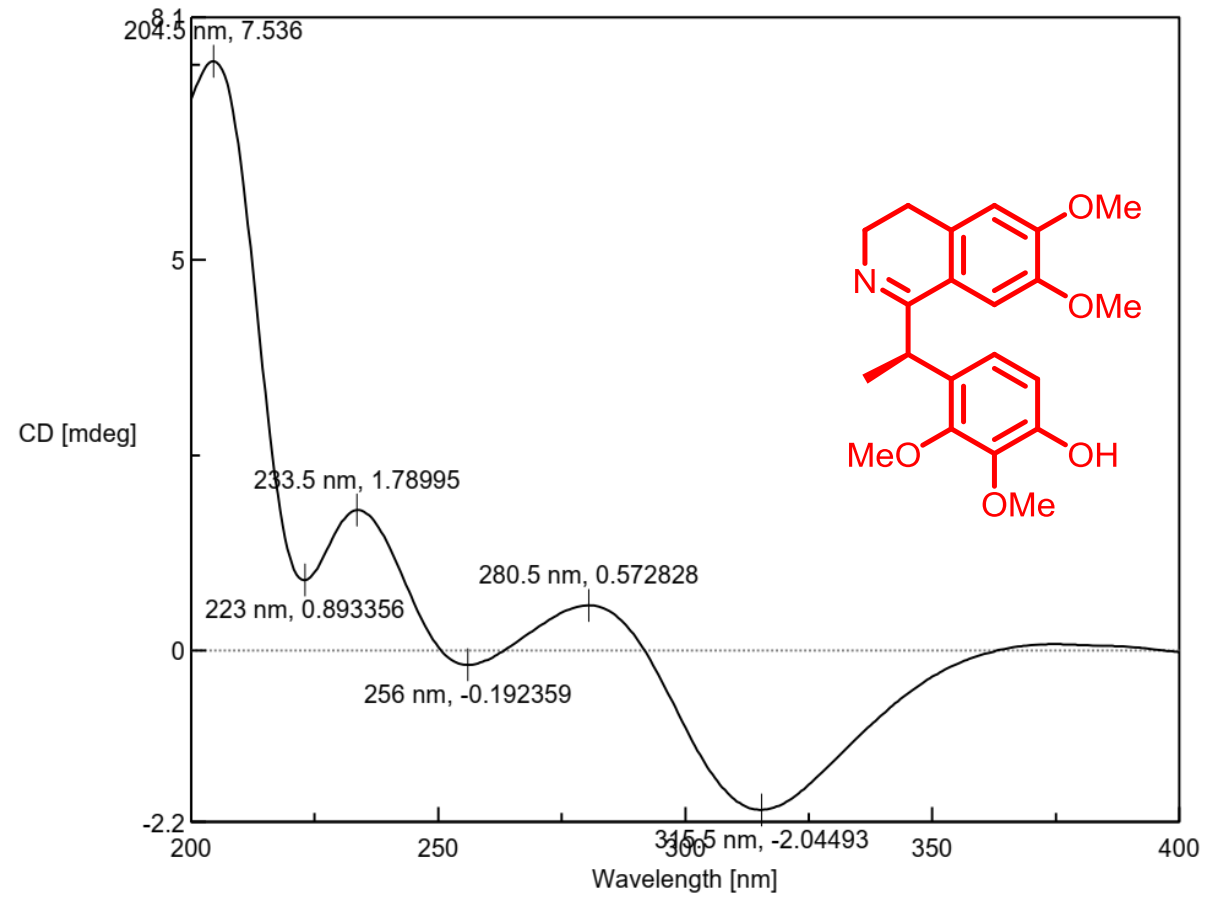

Figure S47. The CD spectrum of compound (-)-4 in $\mathrm{MeOH}$ 

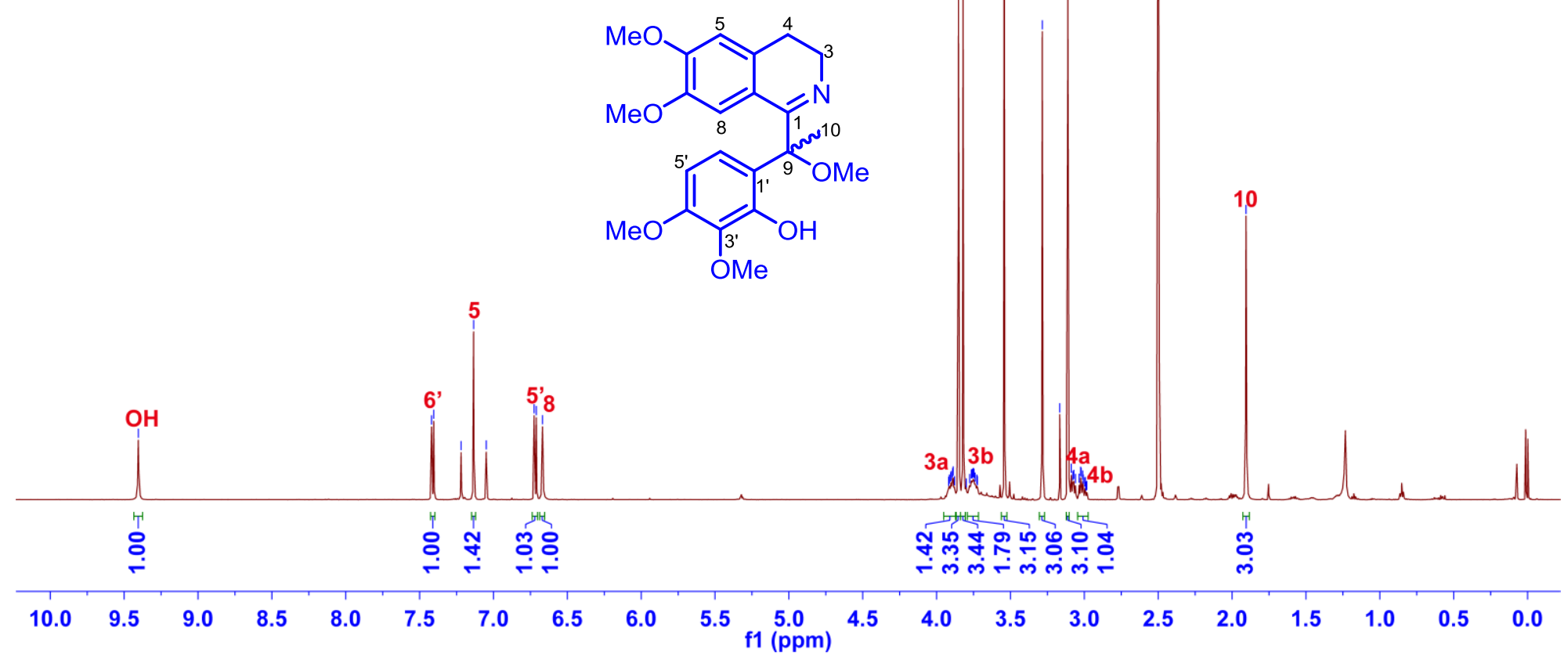

Figure S48. ${ }^{1}$ H NMR spectrum of compound 5 in DMSO- $d_{6}(600 \mathrm{MHz})$ 


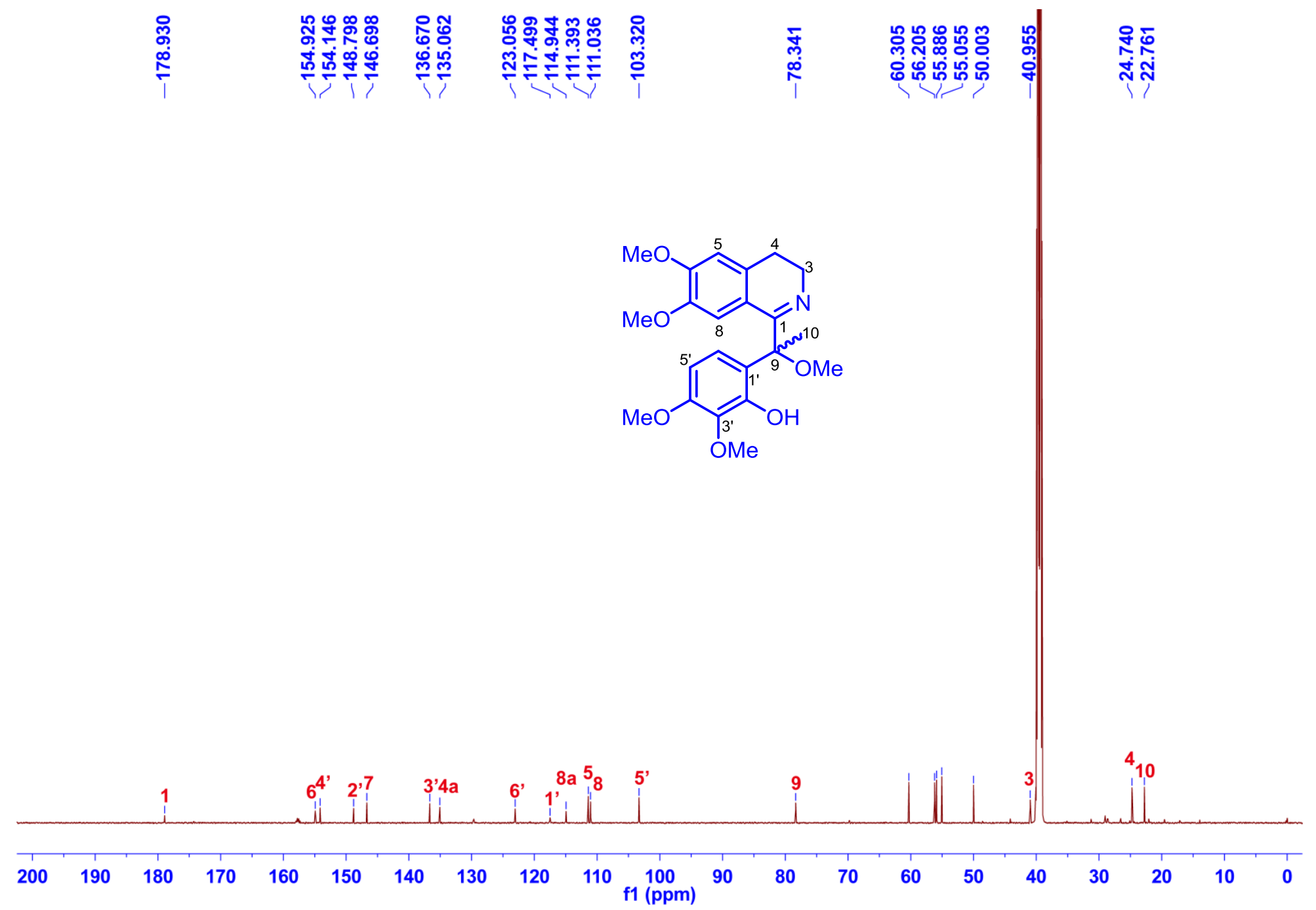

Figure S49. ${ }^{13} \mathrm{C}$ NMR spectrum of compound 5 in DMSO- $d_{6}(150 \mathrm{MHz})$ 


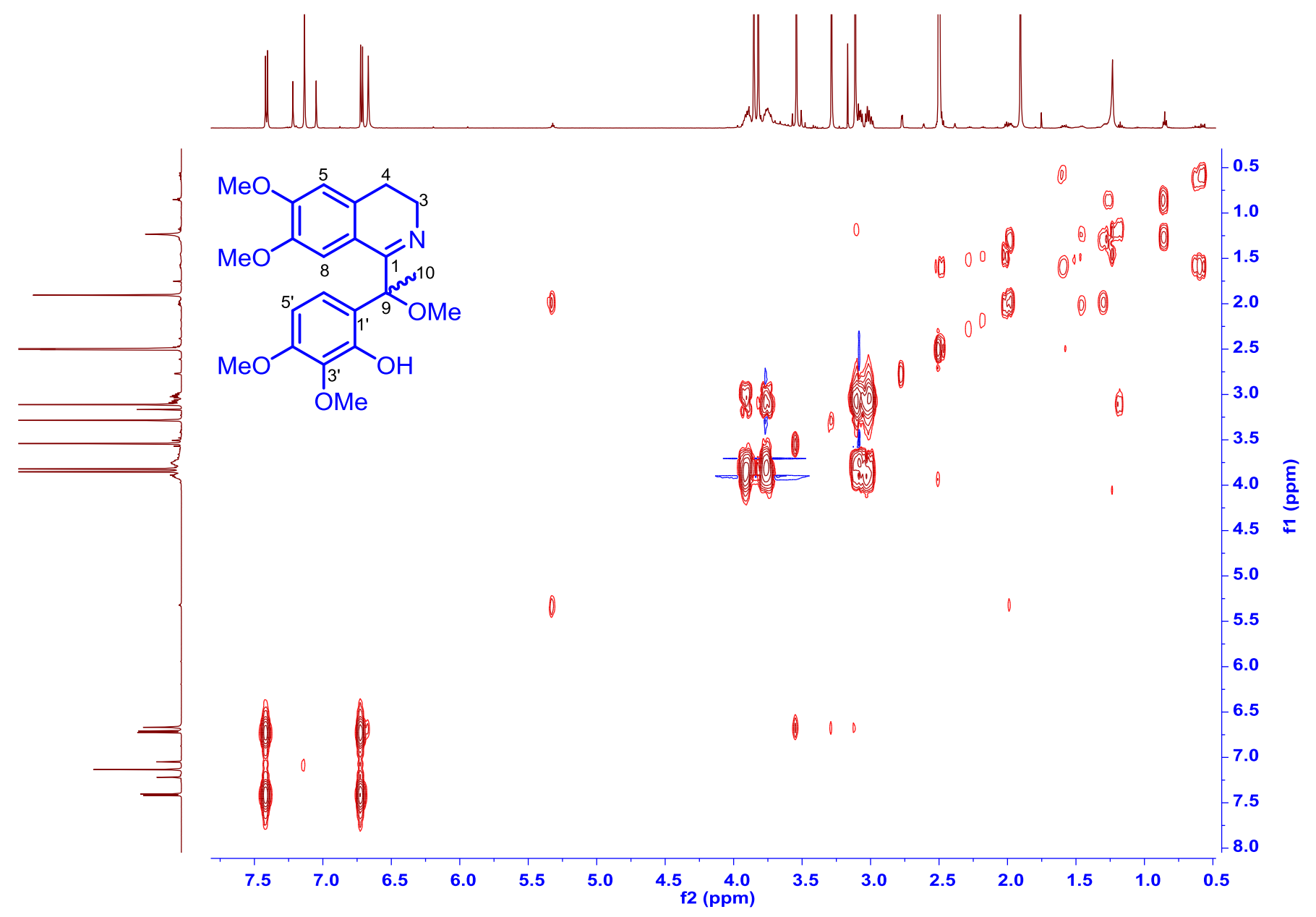

Figure S50. The ${ }^{1} \mathrm{H}-{ }^{1} \mathrm{H}$ COSY spectrum of compound 5 in DMSO-d $6(600 \mathrm{MHz})$ 


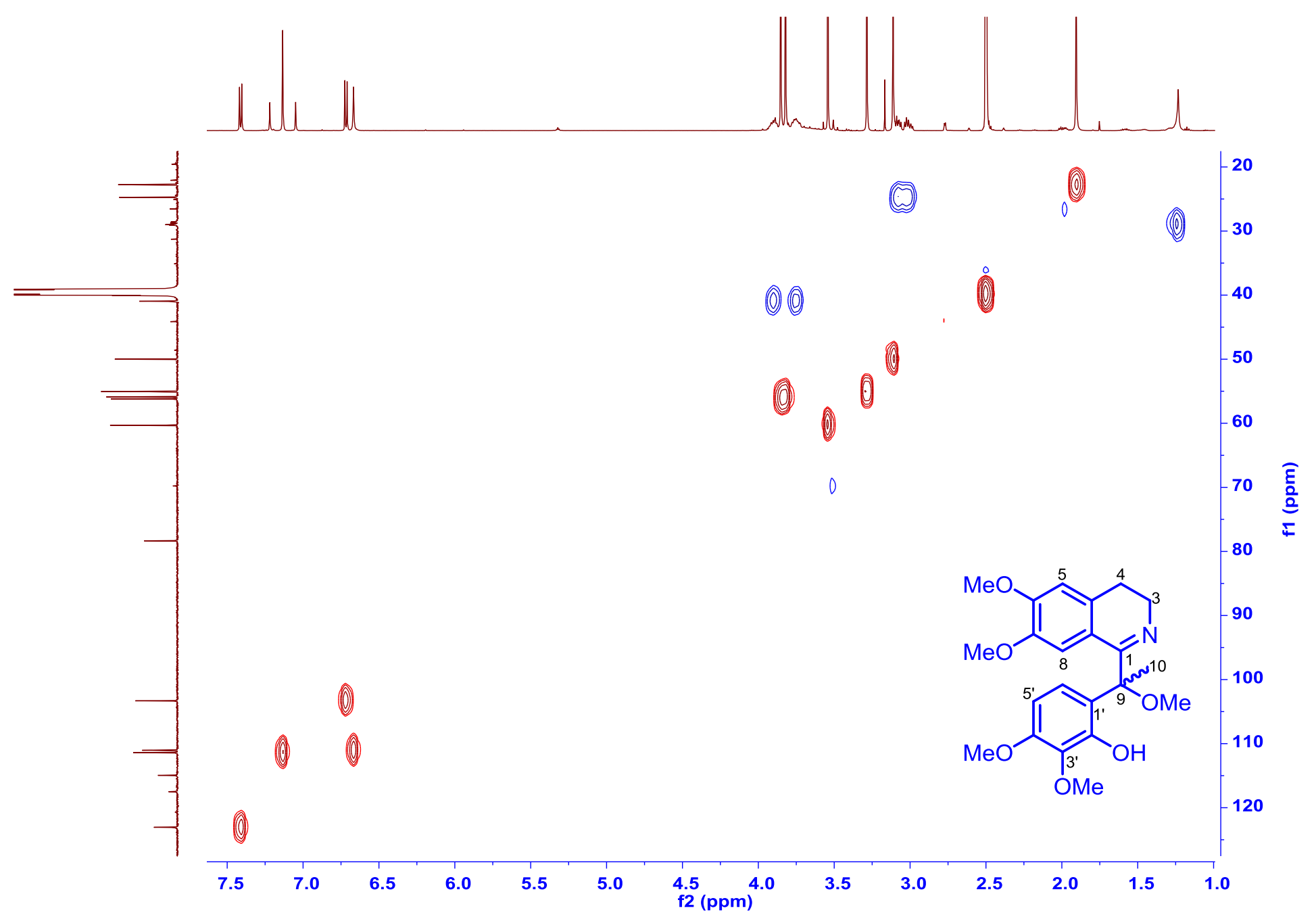

Figure S51. The HSQC spectrum of compound 5 in DMSO-d $d_{6}(600 \mathrm{MHz})$ 


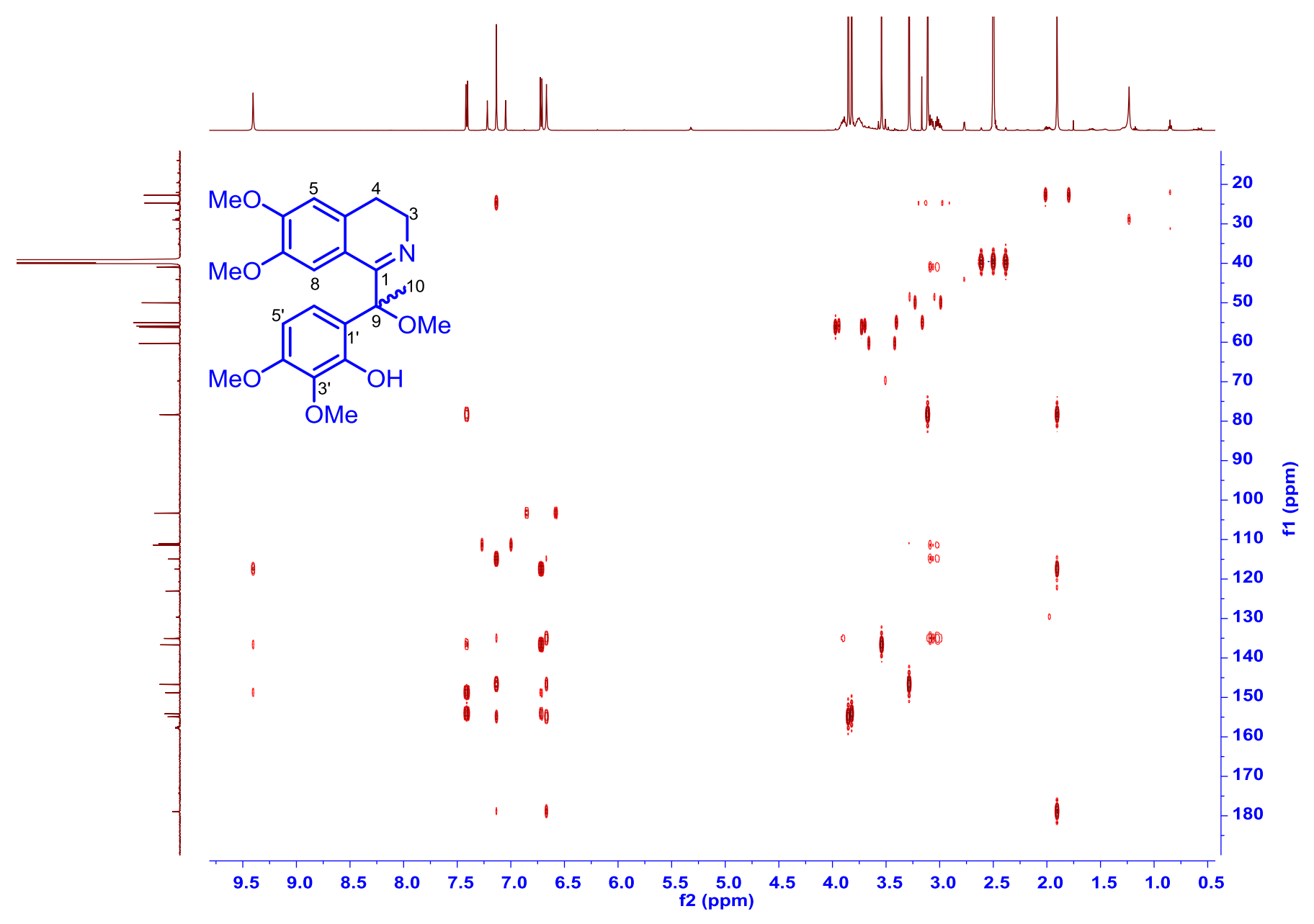

Figure S52. The HMBC spectrum of compound 5 in DMSO- $d_{6}(600 M H z)$ 


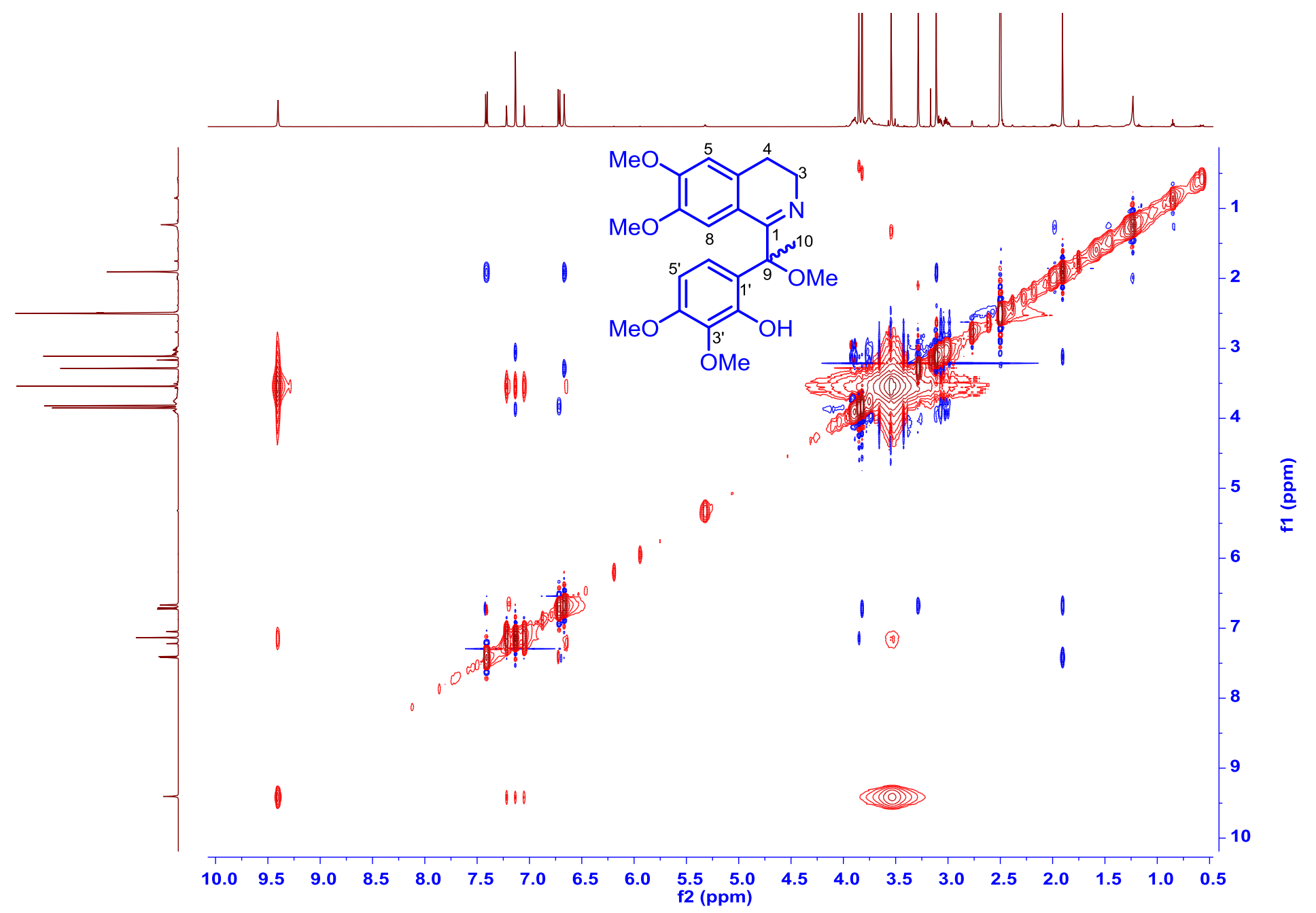

Figure S53. The NOESY spectrum of compound 5 in DMSO- $d_{6}(600 \mathrm{MHz})$ 


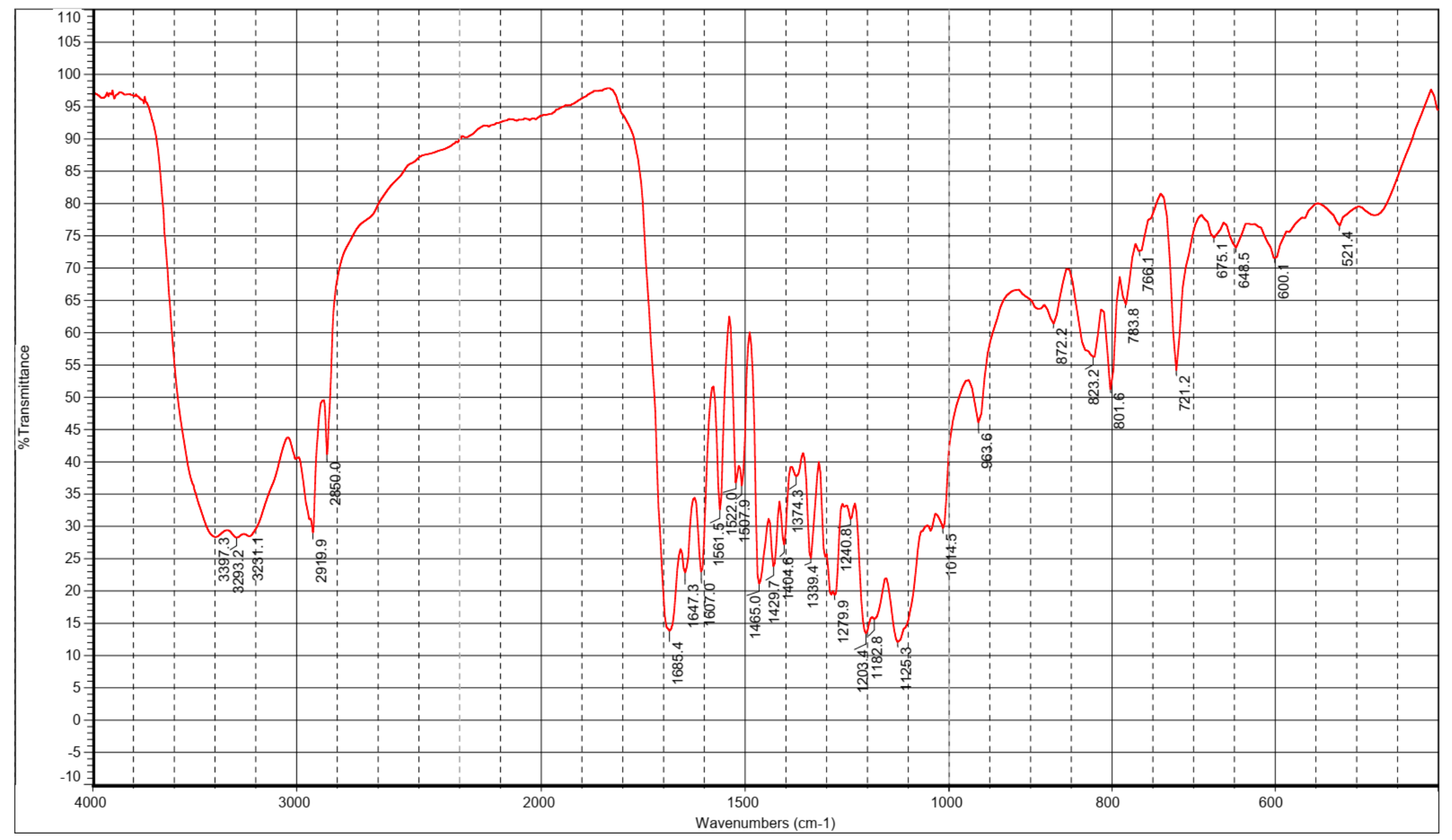

Figure S54. The IR spectrum of compound 5 


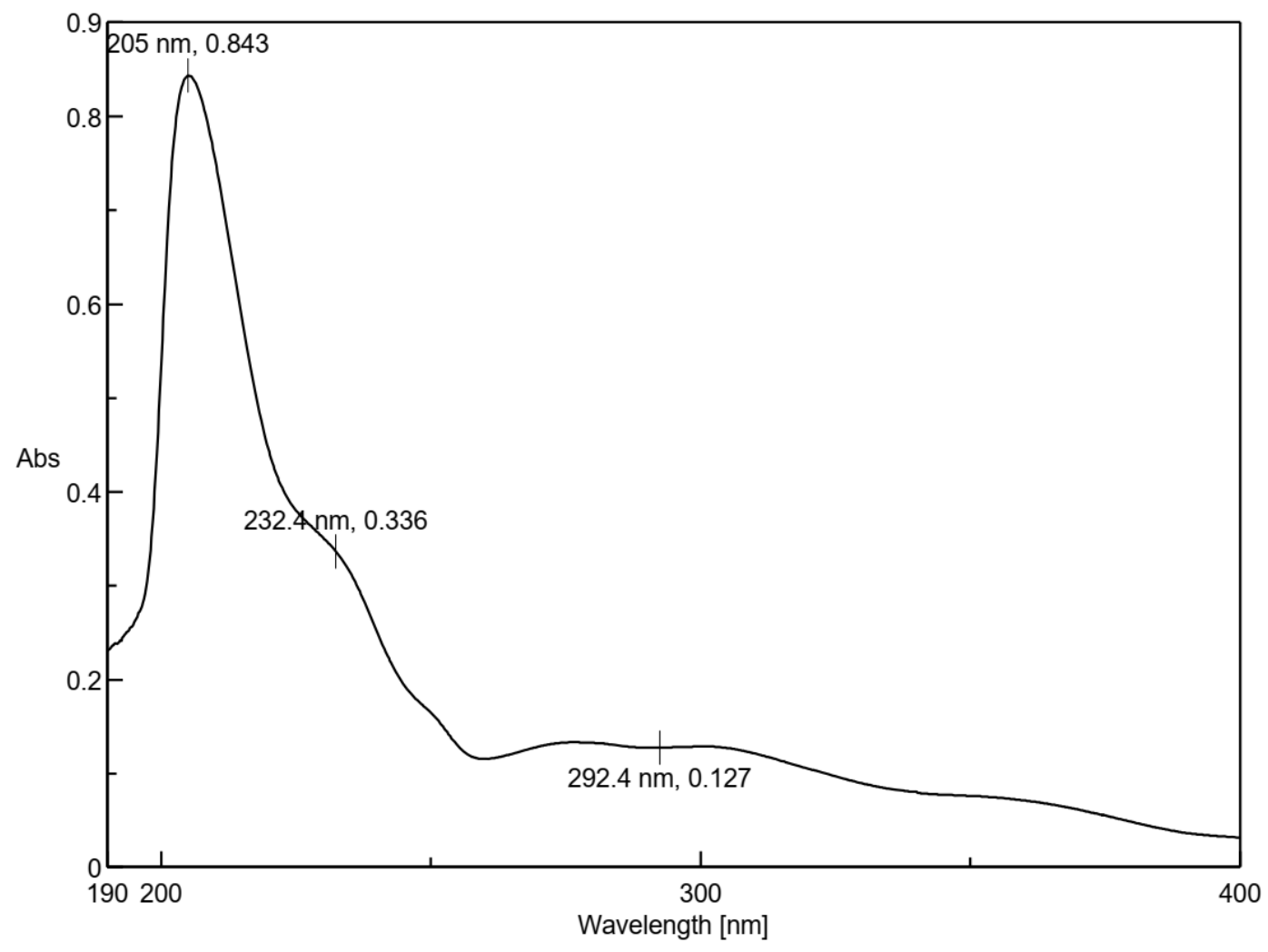

Figure S55.The UV spectrum of compound 5 in $\mathrm{MeOH}$

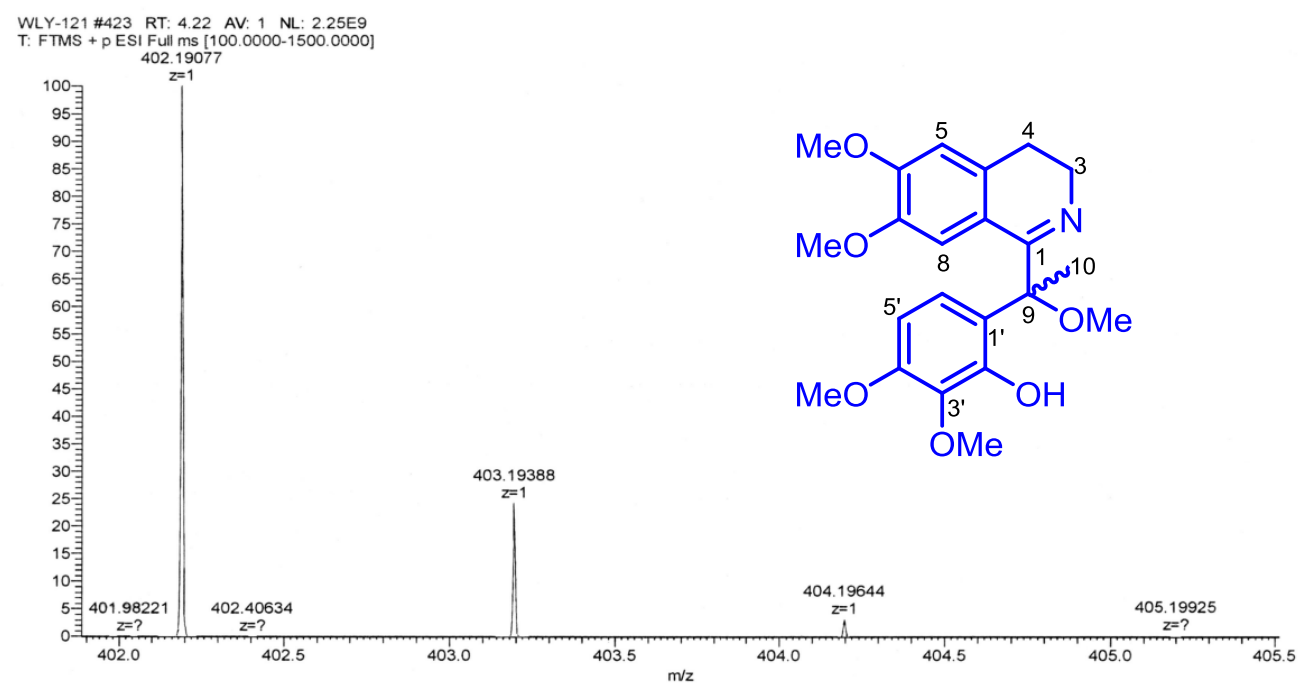

\begin{tabular}{|l|r|r|r|l|l|}
\hline $\mathrm{m} / \mathrm{z}$ & Theo. Mass & Delta (ppm) & RDB equiv. & Composition & \\
\hline & & & & & \\
\hline 402.19077 & 402.19111 & -0.86 & 9.5 & $\mathrm{C} 22 \mathrm{H} 28 \mathrm{O} 6 \mathrm{~N}$ & $\mathrm{M}+\mathrm{H}$ \\
\hline
\end{tabular}

Figure S56.The HR-Mass spectrum of compound 5 in $\mathrm{MeOH}$ 


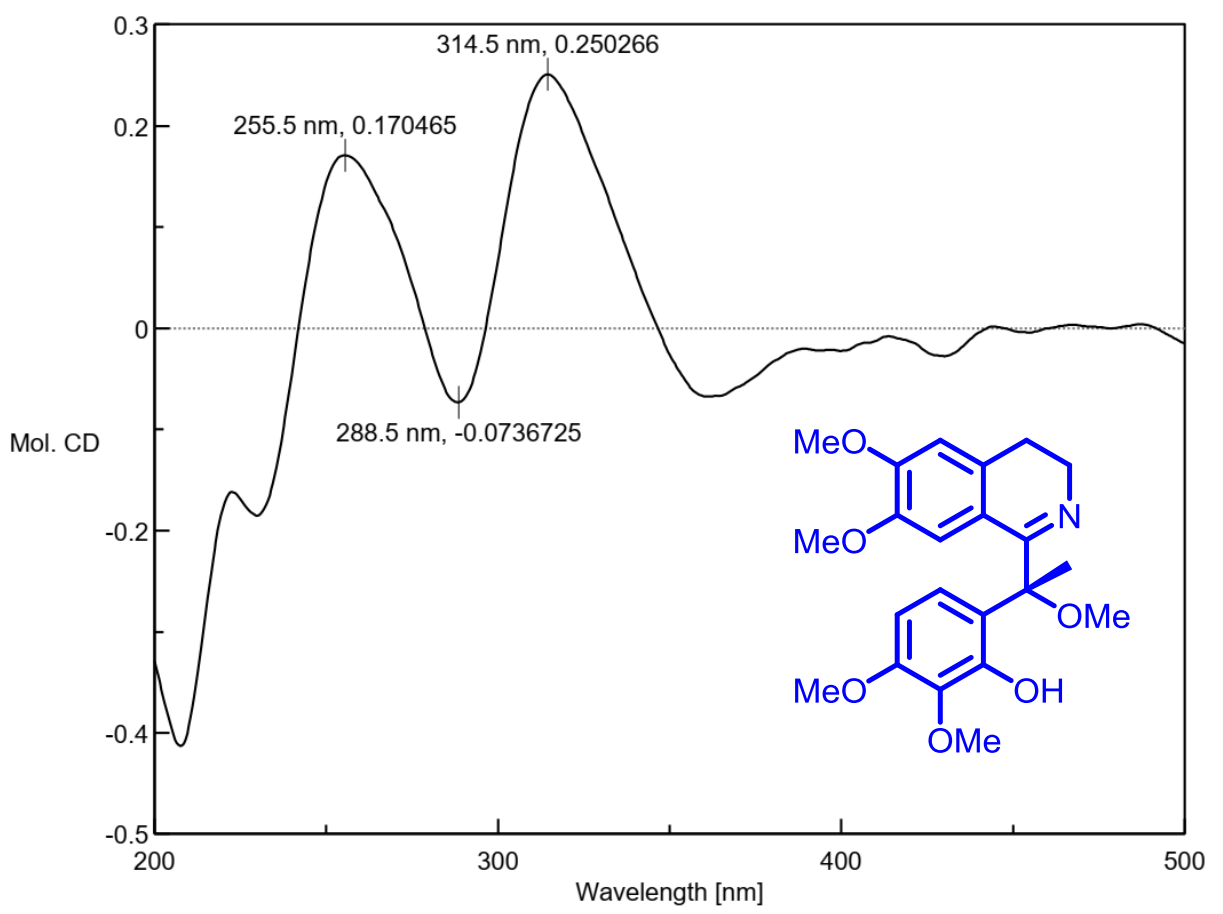

Figure S57. The CD spectrum of compound (+)-5 in $\mathrm{MeOH}$

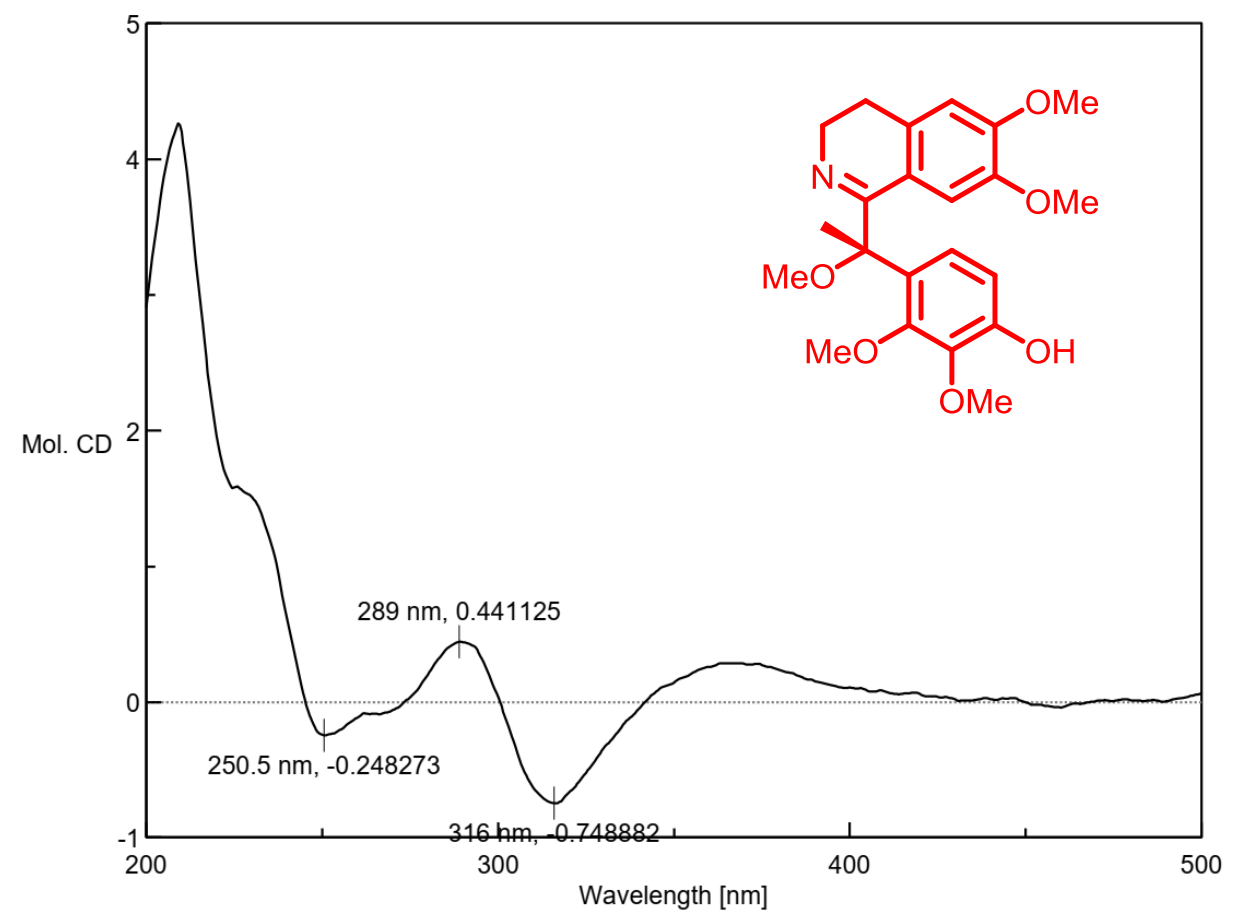

Figure S58. The CD spectrum of compound (-)-5 in MeOH 


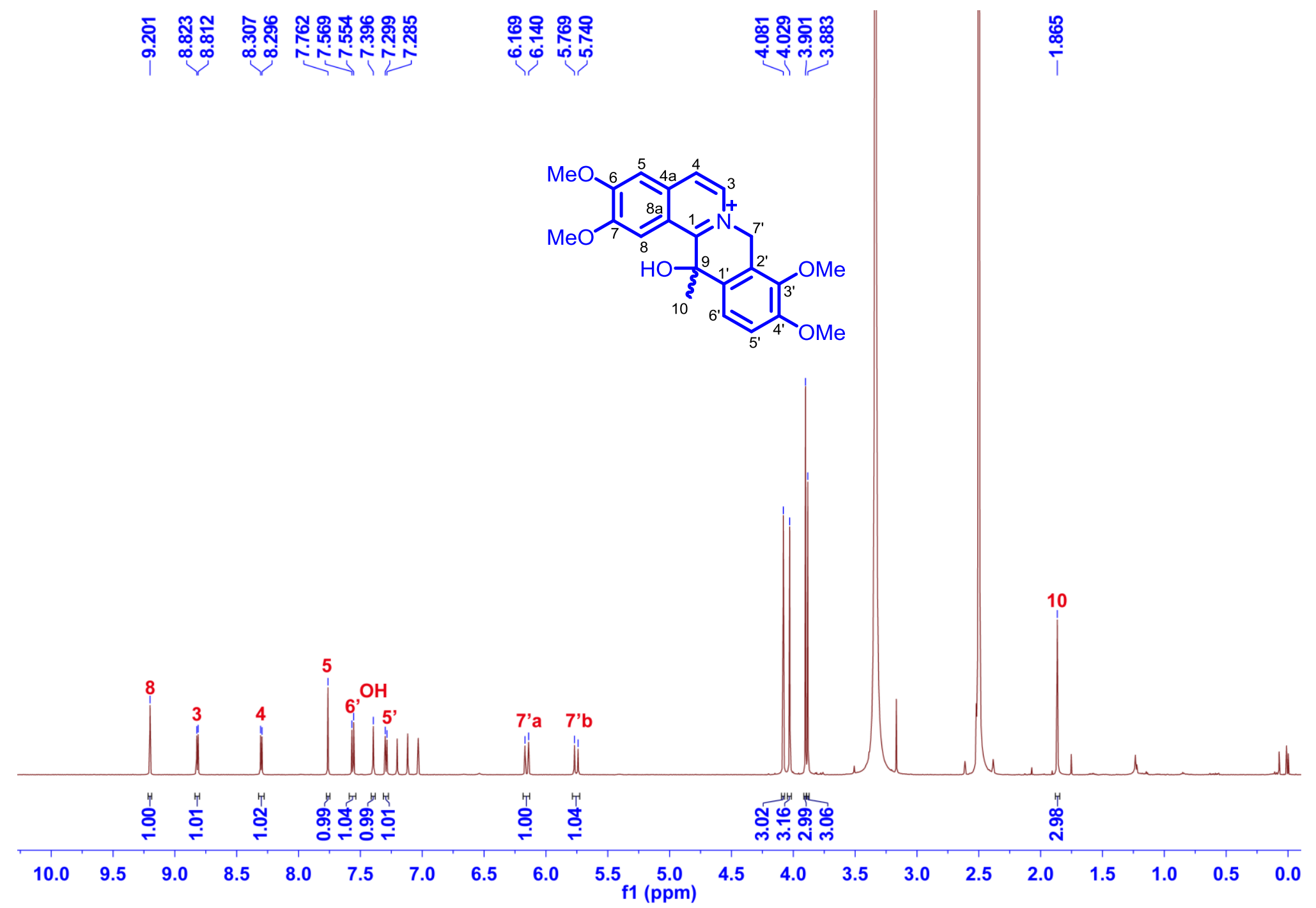

Figure S59. ${ }^{1}$ H NMR spectrum of compound 6 in DMSO-d $6(600 \mathrm{MHz})$ 


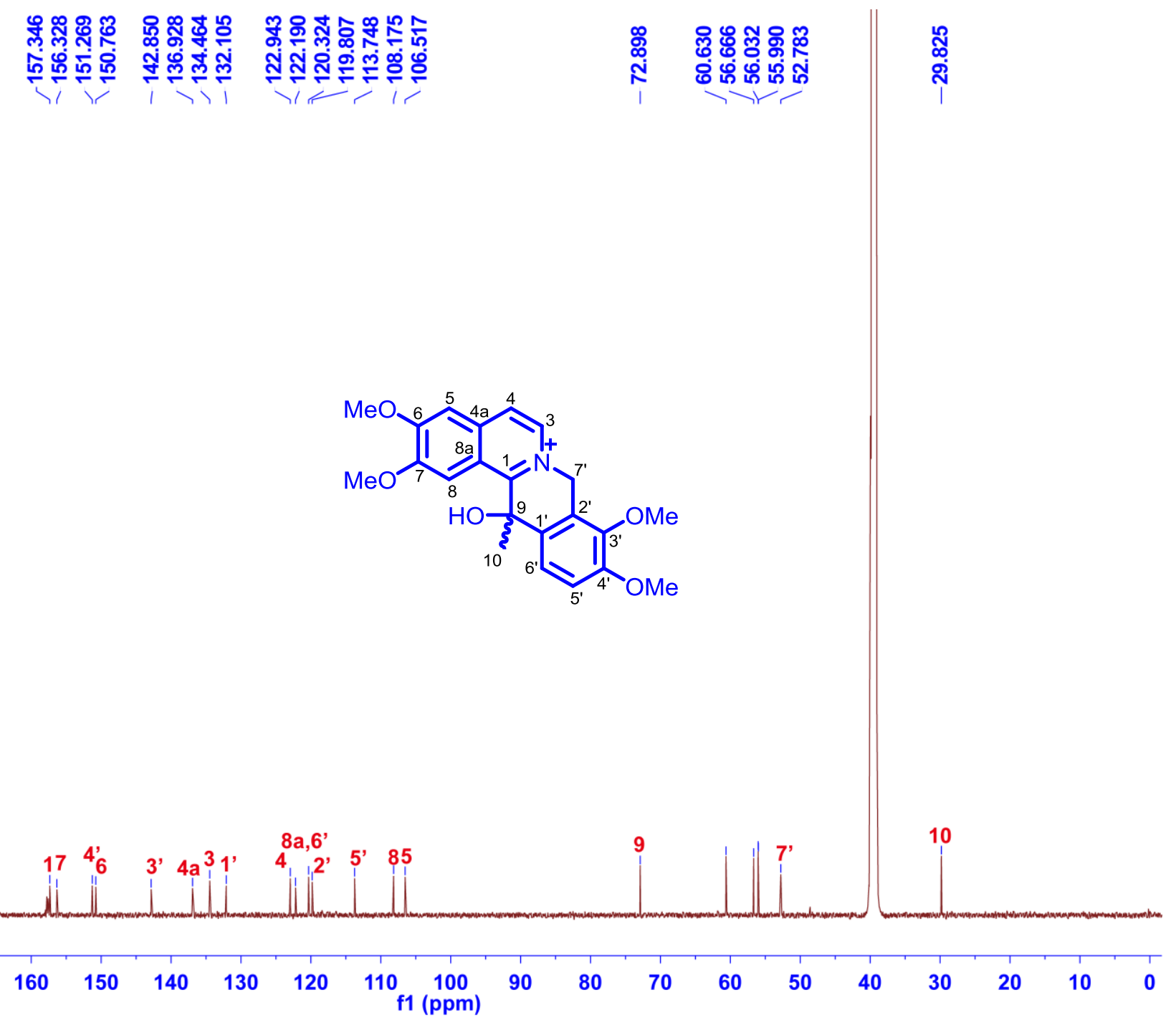

Figure S60. ${ }^{13} \mathrm{C}$ NMR spectrum of compound 6 in DMSO-d $6(150 \mathrm{MHz})$ 


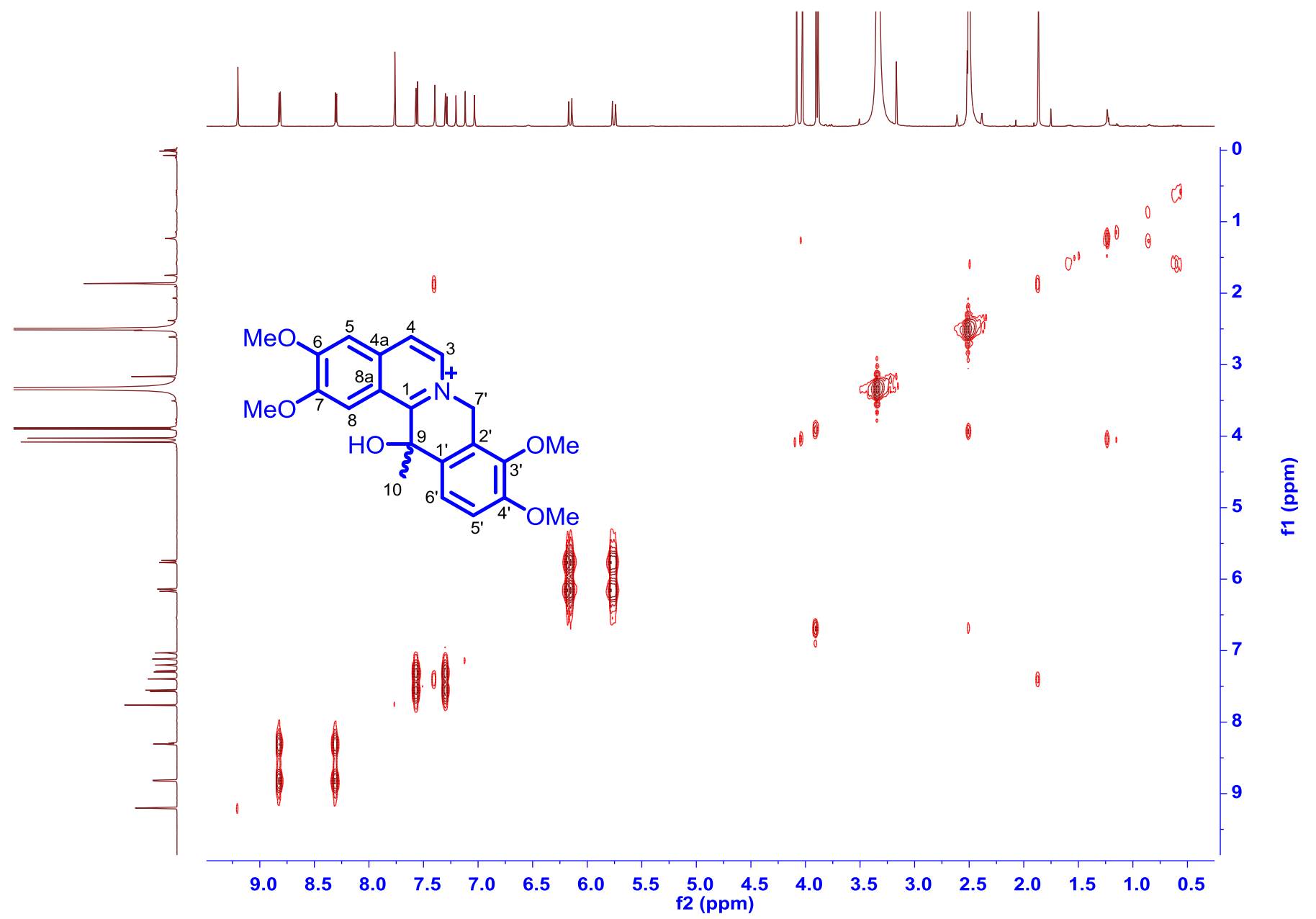

Figure S61. The ${ }^{1} \mathrm{H}-{ }^{1} \mathrm{H}$ COSY spectrum of compound 6 in DMSO-d $6(600 \mathrm{MHz})$ 


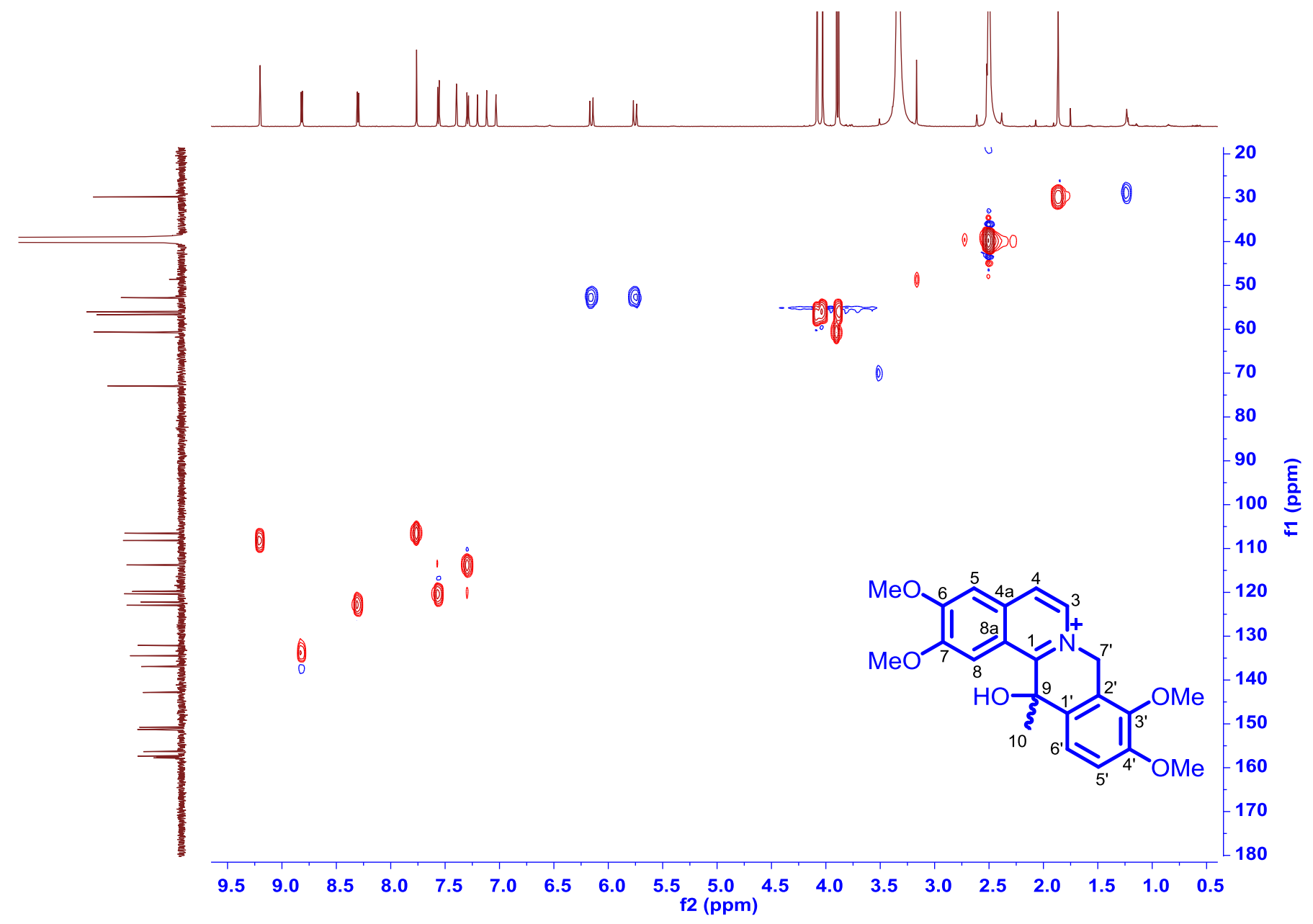

Figure S62. The HSQC spectrum of compound 6 in DMSO- $d_{6}(600 \mathrm{MHz})$ 


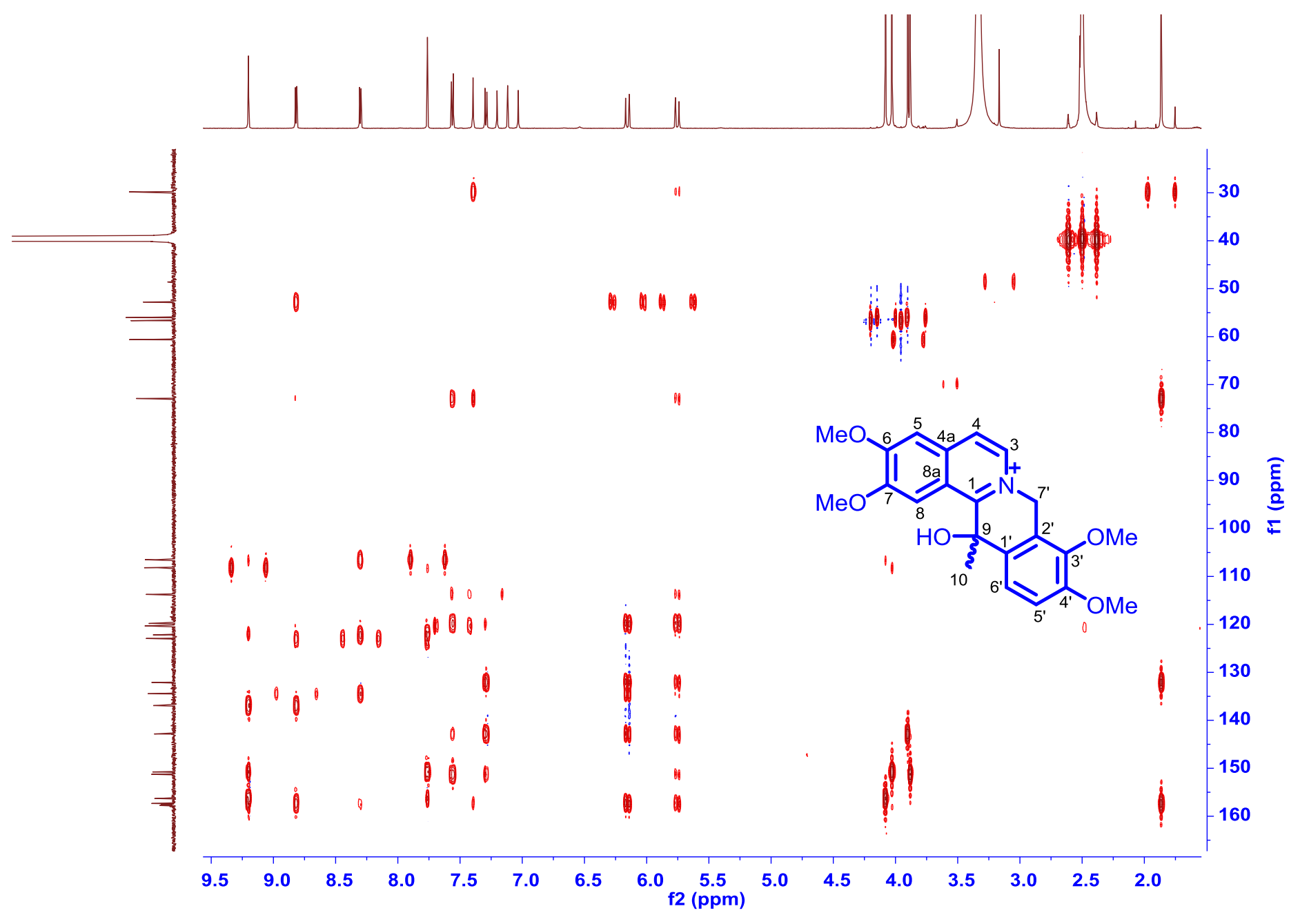

Figure S63. The HMBC spectrum of compound 6 in DMSO-d $6(600 M H z)$ 


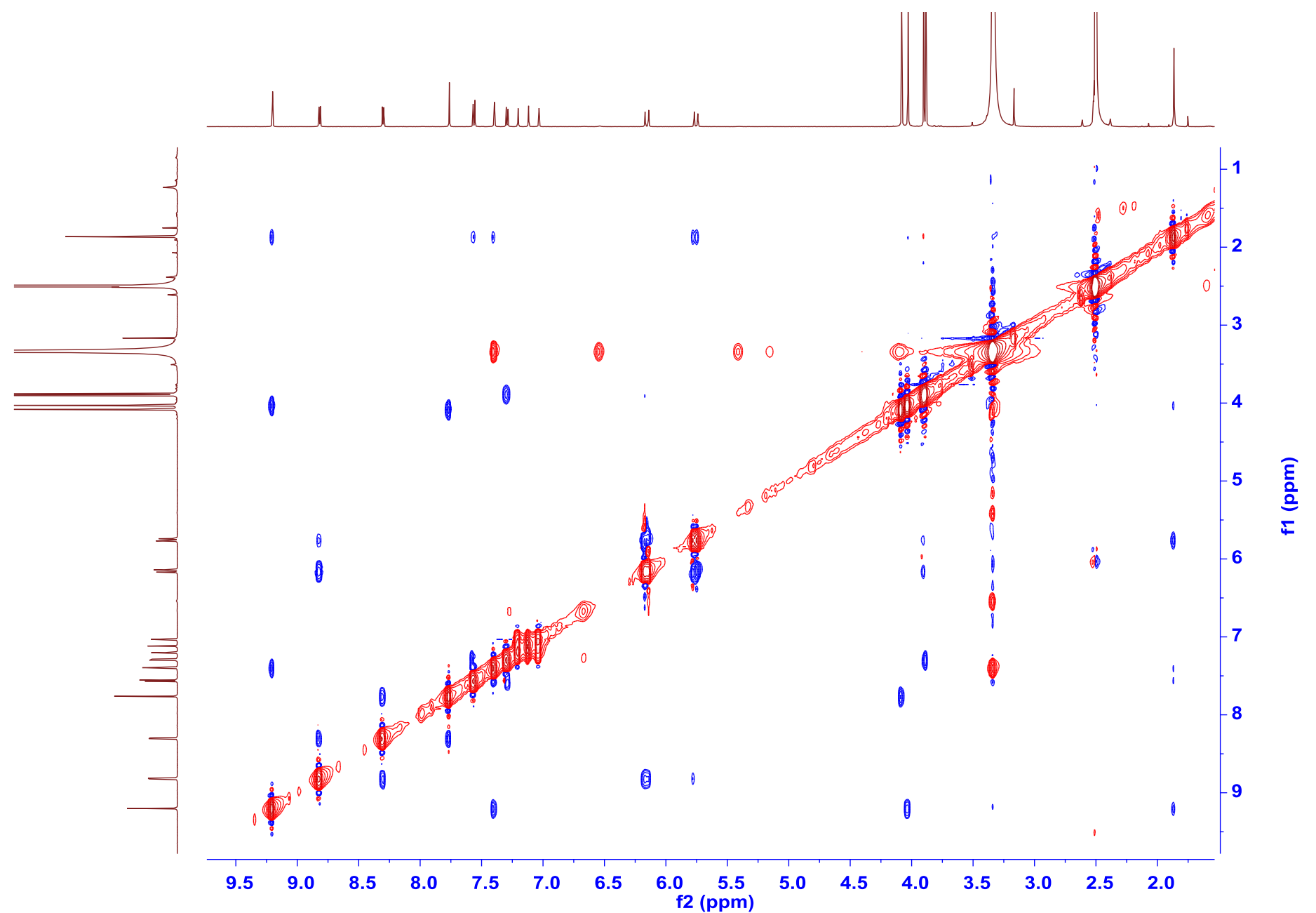

Figure S64. The NOESY spectrum of compound 6 in DMSO-d $(600 \mathrm{MHz})$ 


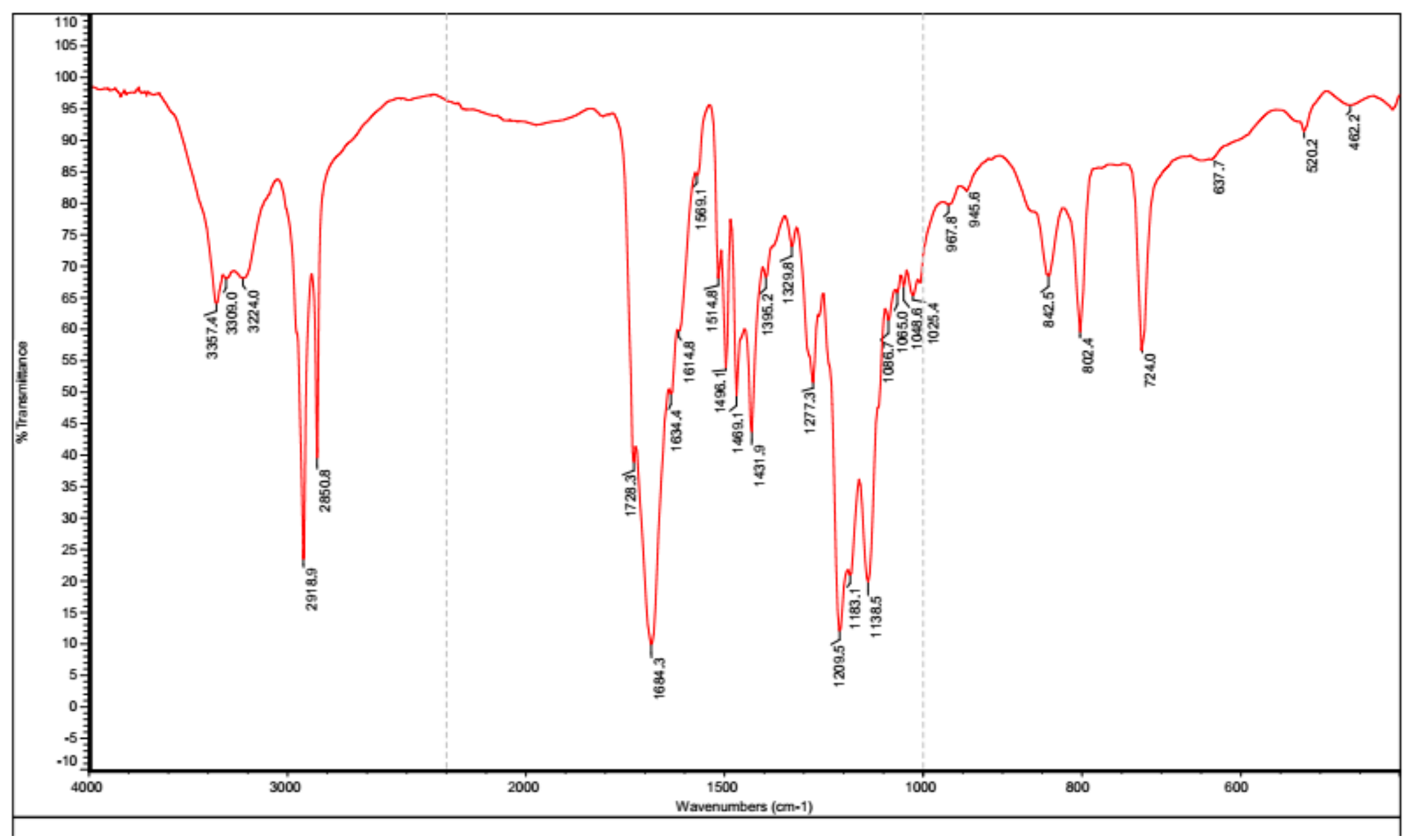

Figure S65. The IR spectrum of compound 6 


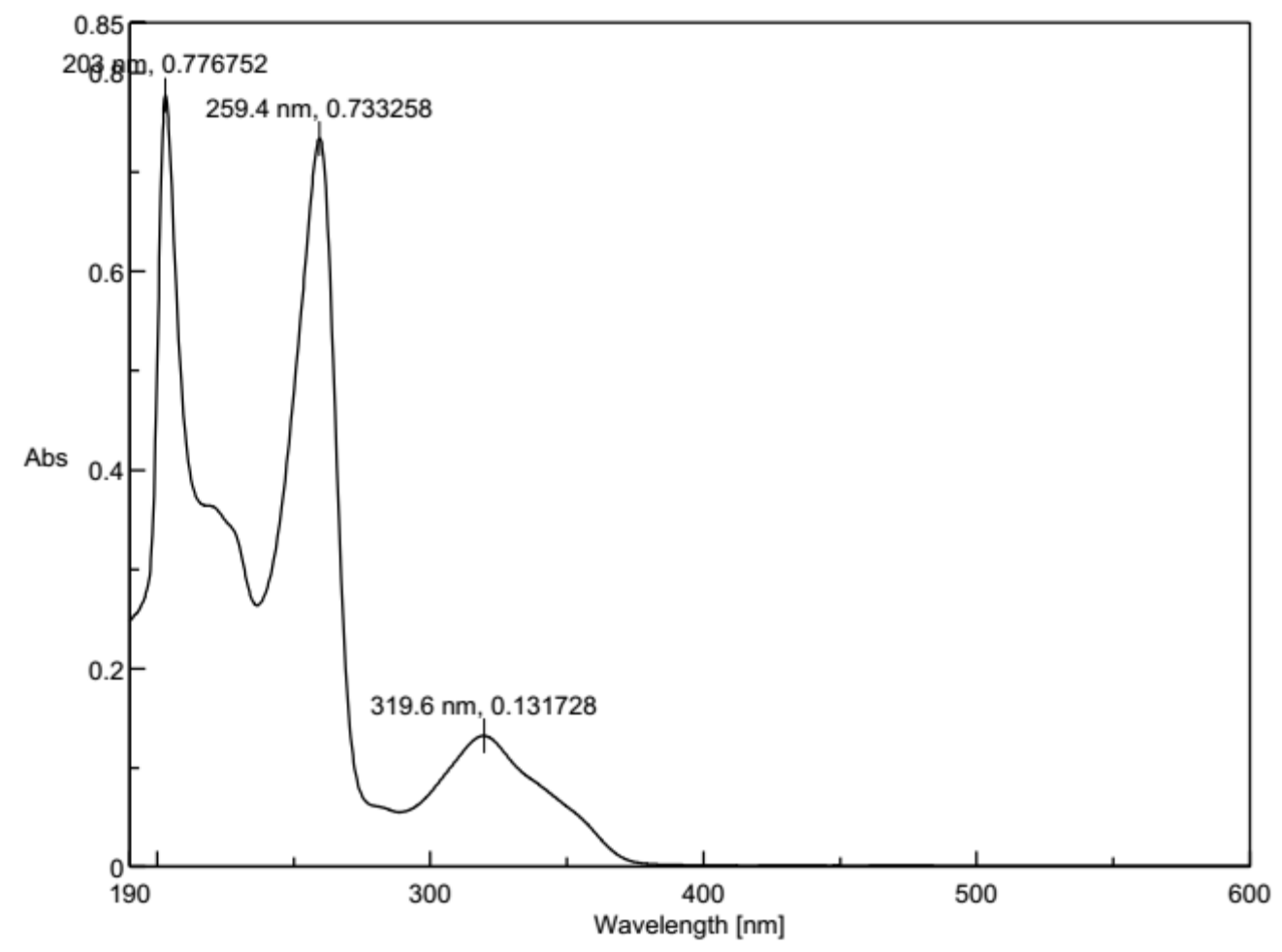

Figure S66. The UV spectrum of compound 6 in $\mathrm{MeOH}$

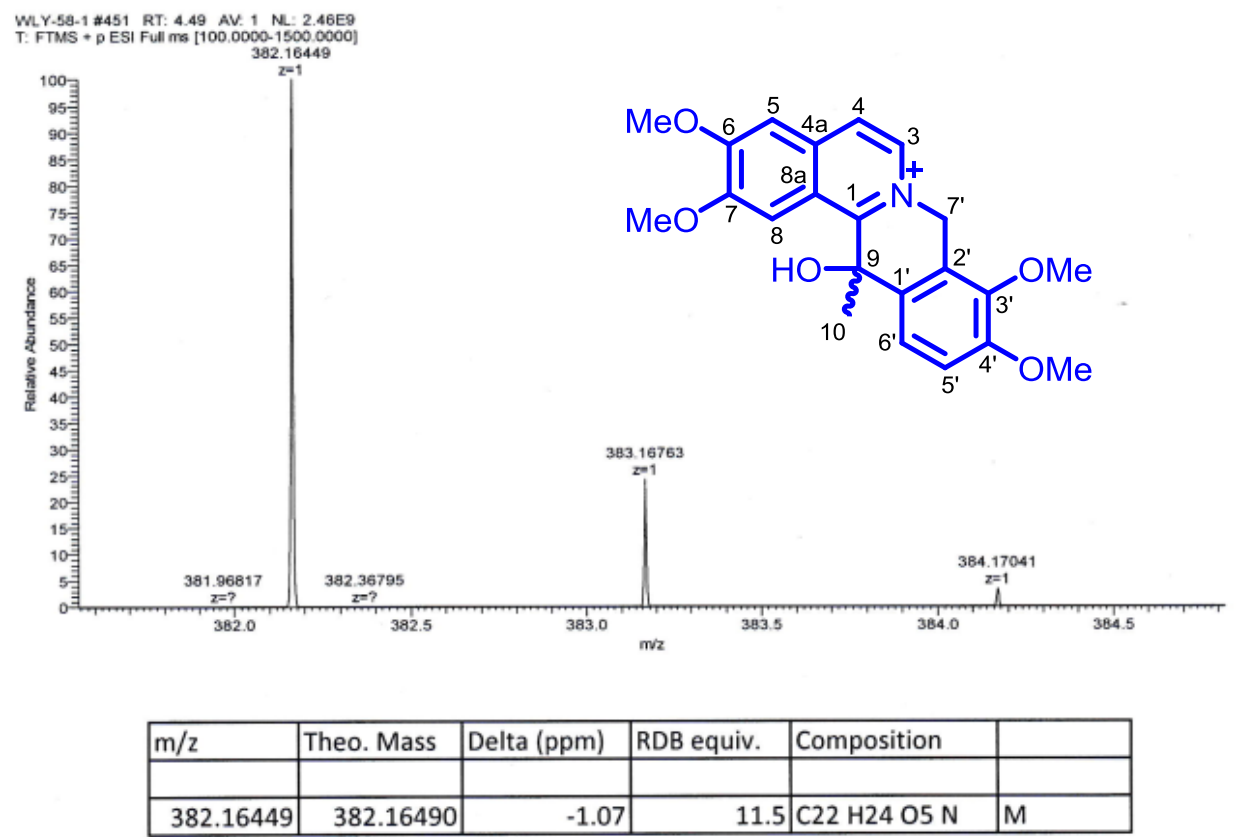

Figure S67. The HR-Mass spectrum of compound 6 in $\mathrm{MeOH}$ 


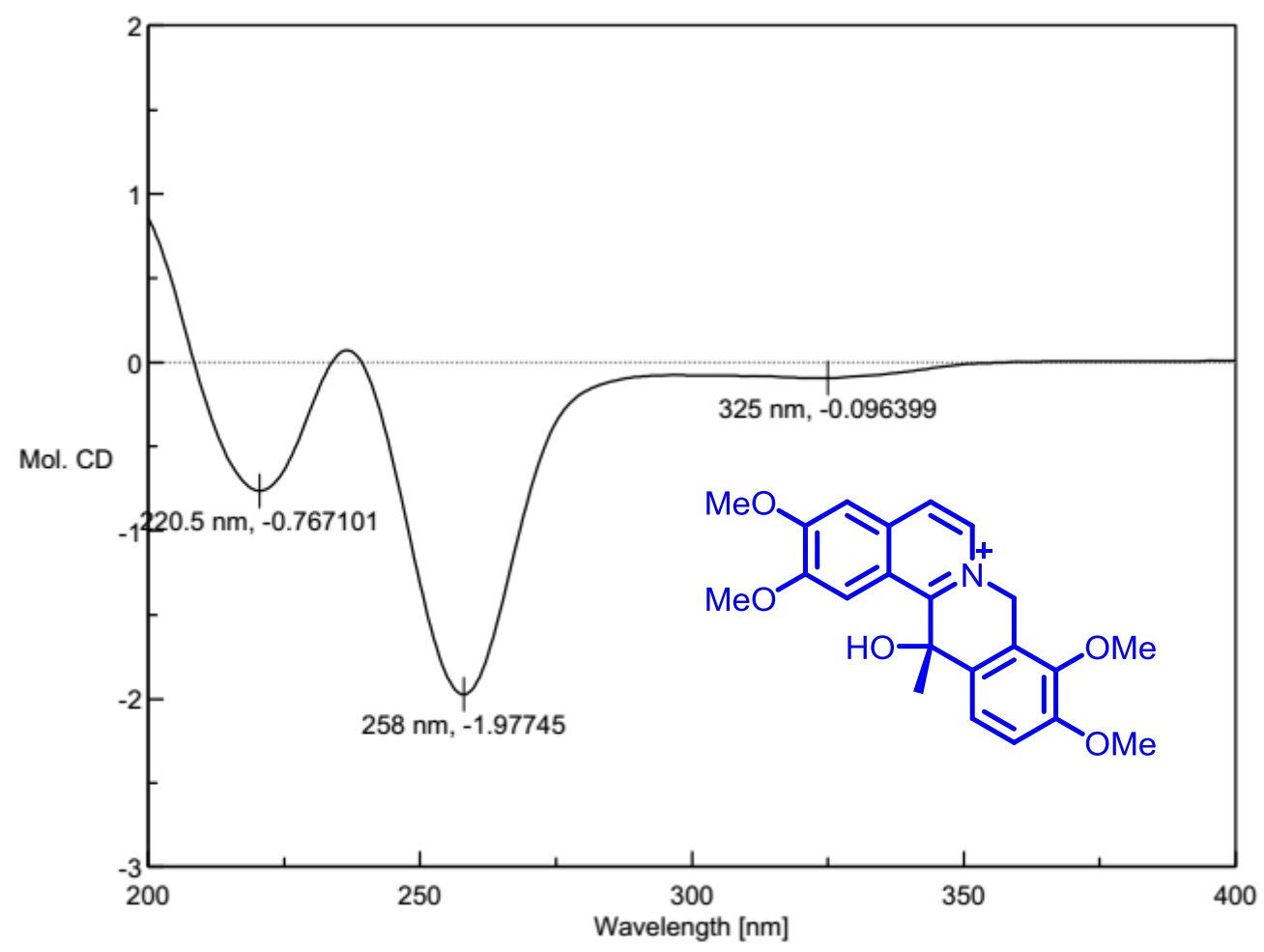

Figure S68. The CD spectrum of compound (+)-6 in MeOH

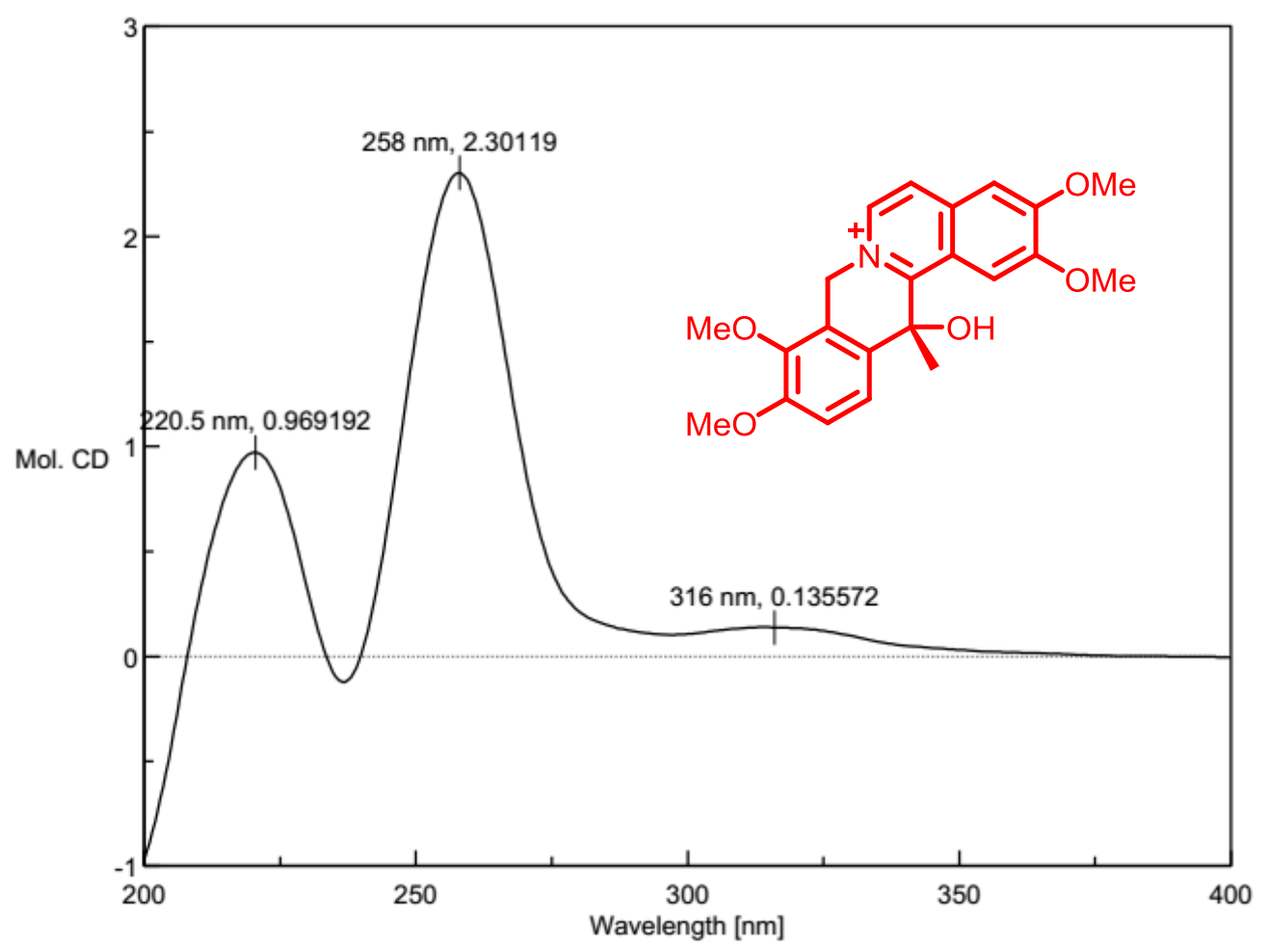

Figure S69. The CD spectrum of compound (-)-6 in $\mathrm{MeOH}$ 
1. Gaussian 16, Revision A.03, M. J. Frisch, G. W. Trucks, H. B. Schlegel, G. E. Scuseria, M. A. Robb, J. R. Cheeseman, G. Scalmani, V. Barone, G. A. Petersson, H. Nakatsuji, X. Li, M. Caricato, A. V. Marenich, J. Bloino, B. G. Janesko, R. Gomperts, B. Mennucci, H. P. Hratchian, J. V. Ortiz, A. F. Izmaylov, J. L. Sonnenberg, D. Williams-Young, F. Ding, F. Lipparini, F. Egidi, J. Goings, B. Peng, A. Petrone, T. Henderson, D. Ranasinghe, V. G. Zakrzewski, J. Gao, N. Rega, G. Zheng, W. Liang, M. Hada, M. Ehara, K. Toyota, R. Fukuda, J. Hasegawa, M. Ishida, T. Nakajima, Y. Honda, O. Kitao, H. Nakai, T. Vreven, K. Throssell, J. A. Montgomery, Jr., J. E. Peralta, F. Ogliaro, M. J. Bearpark, J. J. Heyd, E. N. Brothers, K. N. Kudin, V. N. Staroverov, T. A. Keith, R. Kobayashi, J. Normand, K. Raghavachari, A. P. Rendell, J. C. Burant, S. S. Iyengar, J. Tomasi, M. Cossi, J. M. Millam, M. Klene, C. Adamo, R. Cammi, J. W. Ochterski, R. L. Martin, K. Morokuma, O. Farkas, J. B. Foresman, and D. J. Fox, Gaussian, Inc., Wallingford CT, 2016.

2. N. Grimblat, M. M. Zanardi, A. M. Sarotti, J. Org. Chem. 2015, 80, 12526-12534.

3. T. Bruhn, A. Schaumlöffel, Y. Hemberger, G. Pescitelli, SpecDis version 1.71, Berlin, Germany, 2017, http:/specdis-software.jimdo.com. 